\title{
Synthesis of New Mixed Phosphine Iminophosphorane Bidentate Ligands and their Coordination to Group 10 Metal Centers
}

Leïla Boubekeur, ${ }^{a}$ Louis Ricard, ${ }^{a}$ Nicolas Mézailles, ${ }^{a} *$ Pascal Le Floch ${ }^{\mathrm{a}} *$

\author{
${ }^{a}$ Laboratoire « Hétéroéléments et Coordination », UMR CNRS 7653 (DCPH), Département \\ de Chimie, Ecole Polytechnique, \\ 92128 Palaiseau Cédex, France \\ Tel: +33169334570 ; Fax: +3316933 3990 \\ E-mail: lefloch@poly.polytechnique.fr
}


Figure S1: Molecular structure of 5a

1 page

Table S1-1: Crystal data and structure refinement for 5a

1 page

Table S1-2: Atomic Coordinates and equivalent isotropic displacement parameters for $\mathbf{5 a}$

Table S1-3: Bond lengths $(\AA)$ and angles $\left(^{\circ}\right)$ for $5 \mathbf{a}$

1 page

2 pages

1 page

Table S1-5: Coordinates and equivalent isotropic

displacement parameters for $\mathbf{5 a}$

1 page

Figure S2: Molecular structure of 5c

Table S2-1: Crystal data and structure refinement for 5c

1 page

1 page

Table S2-2: Atomic Coordinates and equivalent isotropic displacement parameters for $\mathbf{5 c}$

Table S2-3: Bond lengths $(\AA)$ and angles $\left(^{\circ}\right)$ for 5c

Table S2-4: Anisotropic displacement parameters for 5c

Table S2-5: Coordinates and equivalent isotropic

displacement parameters for $\mathbf{5 c}$

1 page

1 page

1 page

1 page

Figure S3: Molecular structure of 5e

Table S3-1: Crystal data and structure refinement for 5e

1 page

1 page

Table S3-2: Atomic Coordinates and equivalent isotropic displacement parameters for $\mathbf{5 e}$

Table S3-3: Bond lengths $(\AA)$ and angles $\left({ }^{\circ}\right)$ for $5 e$

1 page

2 pages

Table S3-4: Anisotropic displacement parameters for 5e

1 page

Table S3-5: Coordinates and equivalent isotropic

displacement parameters for $\mathbf{5 e}$

1 page

Figure S4: Molecular structure of 5'e

Table S4-1: Crystal data and structure refinement for 5'e

1 page

1 page

Table S4-2: Atomic Coordinates and equivalent isotropic displacement parameters for 5'e

Table S4-3: Bond lengths $(\AA)$ and angles $\left({ }^{\circ}\right)$ for 5'e

Table S4-4: Anisotropic displacement parameters for 5'e

Table S4-5: Coordinates and equivalent isotropic

displacement parameters for $\mathbf{5}$ 'e

1 page

2 pages

1 page

1 page

Figure S5: Molecular structure of $\mathbf{6 c}$

Table S5-1: Crystal data and structure refinement for $\mathbf{6 c}$

1 page

1 page

Table S5-2: Atomic Coordinates and equivalent

isotropic displacement parameters for $\mathbf{6 c}$

Table S5-3: Bond lengths $(\AA)$ and angles $\left(^{\circ}\right)$ for $\mathbf{6 c}$

1 page

1 page

Table S5-4: Anisotropic displacement parameters for $\mathbf{6 c}$

2 pages

Table S5-5: Coordinates and equivalent isotropic

displacement parameters for $\mathbf{6 c}$

2 pages

Figure S6: Molecular structure of $6 \mathbf{e}$

2 pages

Table S6-1: Crystal data and structure refinement for $\mathbf{6 e}$

2 pages

Table S6-2: Atomic Coordinates and equivalent isotropic displacement parameters for $\mathbf{6 e}$

1 page 
Table S6-3: Bond lengths $(\AA)$ and angles $\left(^{\circ}\right)$ for $6 \mathbf{e}$

2 pages

Table S6-4: Anisotropic displacement parameters for $6 \mathbf{e}$

1 page

Table S6-5: Coordinates and equivalent isotropic

displacement parameters for $\mathbf{6 e}$

1 page

Figure S7: Molecular structure of 7a

Table S7-1: Crystal data and structure refinement for 7a

1 page

1 page

Table S7-2: Atomic Coordinates and equivalent isotropic

1 page

Table S7-3: Bond lengths $(\AA)$ and angles $\left(^{\circ}\right)$ for $7 \mathbf{a}$

2 pages

Table S7-4: Anisotropic displacement parameters for $7 \mathbf{a}$

1 page

Table S7-5: Coordinates and equivalent isotropic displacement

parameters for $7 \mathbf{a}$

1 page 
Figure S1: Molecular structure of 5a

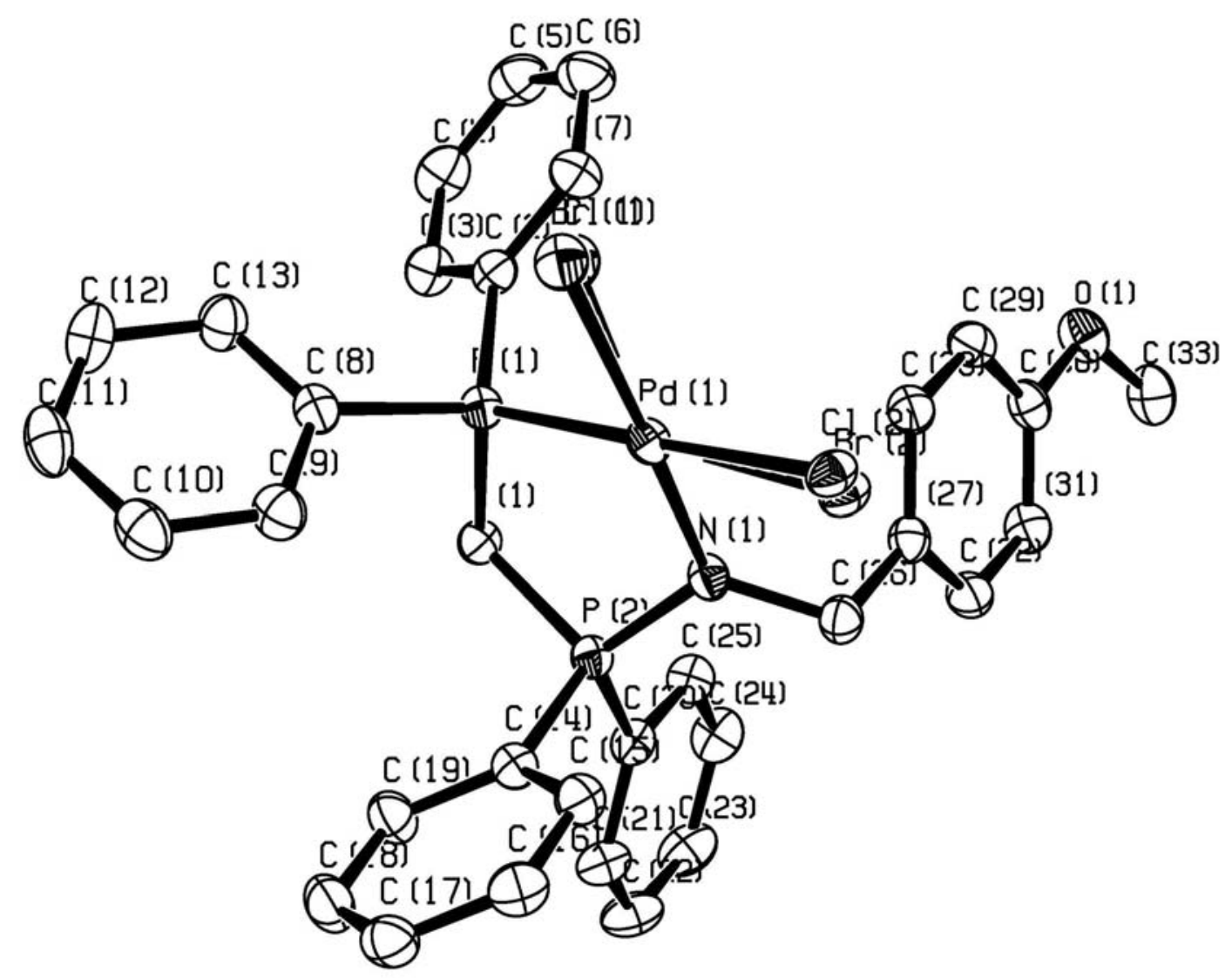


Table S1-1. Crystal data for 5a

Compound

Molecular formula

Molecular weight

Crystal habit

Crystal dimensions(mm)

Crystal system

Space group

$\mathrm{a}(\AA)$

$\mathrm{b}(\AA)$

$\mathrm{c}(\AA)$

$\alpha\left(^{\circ}\right)$

$\beta\left(^{\circ}\right)$

$\gamma\left({ }^{\circ}\right)$

$\mathrm{V}\left(\AA^{3}\right)$

$\mathrm{Z}$

$\mathrm{d}\left(\mathrm{g}-\mathrm{cm}^{-3}\right)$

$\mathrm{F}(000)$

$\mu\left(\mathrm{cm}^{-1}\right)$

Absorption corrections

Diffractometer

$\mathrm{X}$-ray source

$\lambda(\AA)$

Monochromator

$\mathrm{T}(\mathrm{K})$

Scan mode

Maximum $\theta$

HKL ranges

Reflections measured

Unique data

Rint

Reflections used

Criterion

Refinement type

Hydrogen atoms

Parameters refined

Reflections / parameter

wR2

$\mathrm{R} 1$

Weights $a, b$

GoF

difference peak / hole (e $\left.\AA^{-3}\right)$ pdmeobenziminop

$\mathrm{C}_{33} \mathrm{H}_{31} \mathrm{BrClNOP}_{2} \mathrm{Pd}, \mathrm{C}_{4} \mathrm{H}_{8} \mathrm{O}$

813.39

orange block

$0.18 \mathrm{x} 0.18 \mathrm{x} 0.18$

triclinic

Pbar1

9.5450(10)

$10.5560(10)$

$18.1810(10)$

93.1420(10)

$102.6860(10)$

106.8090(10)

$1697.0(3)$

2

1.592

824

1.932

multi-scan ; $0.7224 \min , 0.7224 \max$

KappaCCD

$\mathrm{MoK} \alpha$

0.71069

graphite

150.0(1)

phi and omega scans

30.03

$-813 ;-1413 ;-2522$

13220

9834

0.0150

8631

I $>2 \sigma \mathrm{I})$

Fsqd

mixed

414

20

0.0863

0.0312

$0.0395 ; 1.5250$

1.021

$1.436(0.079) /-0.955(0.079)$ 
Table S1-2. Atomic Coordinates (A x 10^4) and equivalent isotropic displacement parameters $\left(A^{\wedge} 2 \times 10^{\wedge} 3\right)$ for $5 a$

\begin{tabular}{|c|c|c|c|c|}
\hline atom & $\mathrm{x}$ & $\mathrm{Y}$ & $\mathrm{z}$ & $\mathrm{U}(\mathrm{eq})$ \\
\hline $\mathrm{Pd}(1)$ & $-2263(1)$ & $1176(1)$ & $2732(1)$ & 21 (1) \\
\hline $\operatorname{Br}(1)$ & $-2970(5)$ & $-1011(2)$ & $2015(2)$ & $27(1)$ \\
\hline $\operatorname{Br}(2)$ & $-3759(3)$ & $300(2)$ & $3654(1)$ & $23(1)$ \\
\hline $\mathrm{Cl}(2)$ & $-3794(8)$ & $118(5)$ & $3553(3)$ & $23(1)$ \\
\hline $\mathrm{Cl}(1)$ & $-3050(10)$ & $-868(6)$ & $2001(6)$ & $27(1)$ \\
\hline $\mathrm{P}(1)$ & $-814(1)$ & $1963(1)$ & $1954(1)$ & $20(1)$ \\
\hline$P(2)$ & $157(1)$ & 3857 (1) & $3350(1)$ & $20(1)$ \\
\hline$O(1)$ & $-6701(2)$ & $5822(2)$ & $1992(1)$ & $33(1)$ \\
\hline $\mathrm{N}(1)$ & $-1604(2)$ & $3121(2)$ & $3263(1)$ & $22(1)$ \\
\hline$C(1)$ & $430(2)$ & 3645 (2) & 2405 (1) & $22(1)$ \\
\hline$C(2)$ & $-1784(2)$ & $2230(2)$ & $1029(1)$ & $23(1)$ \\
\hline$C(3)$ & $-1014(3)$ & $3121(2)$ & $604(1)$ & $29(1)$ \\
\hline$C(4)$ & $-1776(3)$ & $3334(3)$ & $-97(1)$ & $36(1)$ \\
\hline$C(5)$ & $-3304(3)$ & $2672(3)$ & $-373(1)$ & $37(1)$ \\
\hline$C(6)$ & $-4077(3)$ & $1798(3)$ & $45(1)$ & $37(1)$ \\
\hline$C(7)$ & $-3318(3)$ & $1575(2)$ & $753(1)$ & $29(1)$ \\
\hline$C(8)$ & $528(2)$ & $1113(2)$ & 1805 (1) & $23(1)$ \\
\hline$C(9)$ & 1395 (3) & $782(2)$ & 2447 (1) & $30(1)$ \\
\hline$C(10)$ & $2563(3)$ & $285(2)$ & $2380(2)$ & $34(1)$ \\
\hline$C(11)$ & $2865(3)$ & $102(2)$ & $1680(2)$ & $35(1)$ \\
\hline$C(12)$ & $1978(3)$ & $382(3)$ & $1041(2)$ & $37(1)$ \\
\hline$C(13)$ & $806(3)$ & $890(2)$ & $1100(1)$ & $30(1)$ \\
\hline$C(14)$ & $1399(2)$ & $3160(2)$ & $3980(1)$ & $23(1)$ \\
\hline$C(15)$ & $793(3)$ & $2306(2)$ & 4470 (1) & $26(1)$ \\
\hline$C(16)$ & $1708(3)$ & $1728(2)$ & 4951 (1) & $30(1)$ \\
\hline$C(17)$ & 3205 (3) & 1985 (2) & $4939(1)$ & 31 (1) \\
\hline$C(18)$ & 3811 (3) & $2834(3)$ & $4453(2)$ & $34(1)$ \\
\hline C (19) & $2914(3)$ & $3424(2)$ & $3974(1)$ & $30(1)$ \\
\hline$C(20)$ & $710(2)$ & $5622(2)$ & $3644(1)$ & $24(1)$ \\
\hline$C(21)$ & 1959 (3) & $6280(2)$ & $4230(1)$ & $29(1)$ \\
\hline$C(22)$ & $2362(3)$ & $7658(2)$ & $4404(1)$ & $37(1)$ \\
\hline$C(23)$ & $1517(3)$ & $8368(2)$ & $4002(2)$ & $38(1)$ \\
\hline$C(24)$ & $255(3)$ & $7719(2)$ & $3420(2)$ & 35 (1) \\
\hline$C(25)$ & $-145(3)$ & $6346(2)$ & $3235(1)$ & $28(1)$ \\
\hline$C(26)$ & $-2468(2)$ & $3618(2)$ & $3724(1)$ & $25(1)$ \\
\hline$C(27)$ & $-3582(2)$ & $4220(2)$ & $3262(1)$ & $23(1)$ \\
\hline$C(28)$ & $-4587(3)$ & $3544(2)$ & $2583(1)$ & $27(1)$ \\
\hline C (29) & $-5608(3)$ & $4103(2)$ & $2171(1)$ & $29(1)$ \\
\hline$C(30)$ & $-5636(2)$ & $5359(2)$ & 2432 (1) & $27(1)$ \\
\hline C (31) & $-4636(3)$ & $6053(2)$ & $3103(1)$ & $28(1)$ \\
\hline C (32) & $-3627(3)$ & $5466(2)$ & 3512 (1) & $28(1)$ \\
\hline$C(33)$ & $-6877(3)$ & $7025(3)$ & $2308(2)$ & $37(1)$ \\
\hline$O(2)$ & $-1033(3)$ & $5958(2)$ & $1342(1)$ & $52(1)$ \\
\hline$C(34)$ & $-2643(4)$ & $5484(3)$ & $1216(3)$ & $63(1)$ \\
\hline$C(35)$ & $-3183(5)$ & $6119(4)$ & $494(4)$ & $116(2)$ \\
\hline$C(36)$ & -1901 (5) & $7328(4)$ & $517(2)$ & $66(1)$ \\
\hline$C(37)$ & $-707(4)$ & $7260(3)$ & $1206(2)$ & $53(1)$ \\
\hline
\end{tabular}

$\mathrm{U}(\mathrm{eq})$ is defined as $1 / 3$ the trace of the Uij tensor. 
Table S1-3. Bond lengths (A) and angles (deg) for 5a

$\begin{array}{llll}\mathrm{Pd}(1)-\mathrm{N}(1) & 2.077(2) & \mathrm{Pd}(1)-\mathrm{P}(1) & 2.2197(5) \\ \mathrm{Pd}(1)-\mathrm{C}(1) & 2.301(5) & \mathrm{Pd}(1)-\mathrm{C}(2) & 2.410(6) \\ \mathrm{Pd}(1)-\mathrm{Br}(1) & 2.422(2) & \mathrm{Pd}(1)-\mathrm{Br}(2) & 2.482(2) \\ \mathrm{P}(1)-\mathrm{C}(2) & 1.811(2) & \mathrm{P}(1)-\mathrm{C}(8) & 1.819(2) \\ \mathrm{P}(1)-\mathrm{C}(1) & 1.851(2) & \mathrm{P}(2)-\mathrm{N}(1) & 1.603(2) \\ \mathrm{P}(2)-\mathrm{C}(20) & 1.798(2) & \mathrm{P}(2)-\mathrm{C}(14) & 1.806(2) \\ \mathrm{P}(2)-\mathrm{C}(1) & 1.806(2) & \mathrm{O}(1)-\mathrm{C}(30) & 1.374(3) \\ \mathrm{O}(1)-\mathrm{C}(33) & 1.434(3) & \mathrm{N}(1)-\mathrm{C}(26) & 1.470(3) \\ \mathrm{C}(2)-\mathrm{C}(7) & 1.389(3) & \mathrm{C}(2)-\mathrm{C}(3) & 1.397(3) \\ \mathrm{C}(3)-\mathrm{C}(4) & 1.387(3) & \mathrm{C}(4)-\mathrm{C}(5) & 1.385(4) \\ \mathrm{C}(5)-\mathrm{C}(6) & 1.380(4) & \mathrm{C}(6)-\mathrm{C}(7) & 1.400(3) \\ \mathrm{C}(8)-\mathrm{C}(13) & 1.387(3) & \mathrm{C}(8)-\mathrm{C}(9) & 1.399(3) \\ \mathrm{C}(9)-\mathrm{C}(10) & 1.387(3) & \mathrm{C}(10)-\mathrm{C}(11) & 1.381(4) \\ \mathrm{C}(11)-\mathrm{C}(12) & 1.381(4) & \mathrm{C}(12)-\mathrm{C}(13) & 1.394(3) \\ \mathrm{C}(14)-\mathrm{C}(19) & 1.393(3) & \mathrm{C}(14)-\mathrm{C}(15) & 1.398(3) \\ \mathrm{C}(15)-\mathrm{C}(16) & 1.390(3) & \mathrm{C}(16)-\mathrm{C}(17) & 1.381(3) \\ \mathrm{C}(17)-\mathrm{C}(18) & 1.389(3) & \mathrm{C}(18)-\mathrm{C}(19) & 1.387(3) \\ \mathrm{C}(20)-\mathrm{C}(21) & 1.386(3) & \mathrm{C}(20)-\mathrm{C}(25) & 1.399(3) \\ \mathrm{C}(21)-\mathrm{C}(22) & 1.391(3) & \mathrm{C}(22)-\mathrm{C}(23) & 1.378(4) \\ \mathrm{C}(23)-\mathrm{C}(24) & 1.385(4) & \mathrm{C}(24)-\mathrm{C}(25) & 1.390(3) \\ \mathrm{C}(26)-\mathrm{C}(27) & 1.516(3) & \mathrm{C}(27)-\mathrm{C}(32) & 1.383(3) \\ \mathrm{C}(27)-\mathrm{C}(28) & 1.393(3) & \mathrm{C}(28)-\mathrm{C}(29) & 1.385(3) \\ \mathrm{C}(29)-\mathrm{C}(30) & 1.393(3) & \mathrm{C}(30)-\mathrm{C}(31) & 1.387(3) \\ \mathrm{C}(31)-\mathrm{C}(32) & 1.394(3) & \mathrm{O}(2)-\mathrm{C}(37) & 1.368(4) \\ \mathrm{O}(2)-\mathrm{C}(34) & 1.432(4) & \mathrm{C}(34)-\mathrm{C}(35) & 1.560(7) \\ \mathrm{C}(35)-\mathrm{C}(36) & 1.482(6) & \mathrm{C}(36)-\mathrm{C}(37) & 1.518(5)\end{array}$

$\mathrm{N}(1)-\mathrm{Pd}(1)-\mathrm{P}(1)$

$\mathrm{P}(1)-\mathrm{Pd}(1)-\mathrm{Cl}(1)$

$\mathrm{P}(1)-\mathrm{Pd}(1)-\mathrm{Cl}(2)$

$\mathrm{N}(1)-\mathrm{Pd}(1)-\mathrm{Br}(1)$

$\mathrm{Cl}$ (1) $-\mathrm{Pd}(1)-\mathrm{Br}(1)$

$\mathrm{N}(1)-\mathrm{Pd}(1)-\mathrm{Br}(2)$

$\mathrm{Cl}$ (1) $-\mathrm{Pd}(1)-\mathrm{Br}(2)$

$\operatorname{Br}(1)-\operatorname{Pd}(1)-\operatorname{Br}(2)$

$\mathrm{C}(2)-\mathrm{P}(1)-\mathrm{C}(1)$

$\mathrm{C}(2)-\mathrm{P}(1)-\mathrm{Pd}(1)$

$\mathrm{C}(1)-\mathrm{P}(1)-\mathrm{Pd}(1)$

$\mathrm{N}(1)-\mathrm{P}(2)-\mathrm{C}(14)$

$\mathrm{N}(1)-\mathrm{P}(2)-\mathrm{C}(1)$

$\mathrm{C}(14)-\mathrm{P}(2)-\mathrm{C}(1)$

$\mathrm{C}(26)-\mathrm{N}(1)-\mathrm{P}(2)$

$\mathrm{P}(2)-\mathrm{N}(1)-\mathrm{Pd}(1)$

$C(7)-C(2)-C(3)$

$\mathrm{C}(3)-\mathrm{C}(2)-\mathrm{P}(1)$

$C(5)-C(4)-C(3)$

$C(5)-C(6)-C(7)$

$\mathrm{C}(13)-\mathrm{C}(8)-\mathrm{C}(9)$

$\mathrm{C}(9)-\mathrm{C}(8)-\mathrm{P}(1)$

$C(11)-C(10)-C(9)$

$C(11)-C(12)-C(13)$

$C(19)-C(14)-C(15)$

$\mathrm{C}(15)-\mathrm{C}(14)-\mathrm{P}(2)$

$C(17)-C(16)-C(15)$

$C(19)-C(18)-C(17)$

$C(21)-C(20)-C(25)$

$\mathrm{C}(25)-\mathrm{C}(20)-\mathrm{P}(2)$

$C(23)-C(22)-C(21)$

$\mathrm{C}(23)-\mathrm{C}(24)-\mathrm{C}(25)$

$\mathrm{N}(1)-\mathrm{C}(26)-\mathrm{C}(27)$

C (32) $-\mathrm{C}(27)-\mathrm{C}(26)$

C (29) $-\mathrm{C}(28)-\mathrm{C}(27)$

$\mathrm{O}(1)-\mathrm{C}(30)-\mathrm{C}(31)$

$C(31)-C(30)-C(29)$

$\mathrm{C}(27)-\mathrm{C}(32)-\mathrm{C}(31)$

$\mathrm{O}(2)-\mathrm{C}(34)-\mathrm{C}(35)$
$86.84(5)$

$88.3(3)$

$174.5(1)$

$175.0(1)$

$3.5(3)$

$92.55(6)$

$92.6(3)$

$92.2(1)$

$104.6(1)$

$115.99(7)$

$106.73(7)$

$114.2(1)$

$104.9(1)$

$108.5(1)$

$121.7(1)$

$111.7(1)$

$119.8(2)$

$120.6(2)$

$120.0(2)$

$120.1(2)$

$119.5(2)$

$117.5(2)$

$120.2(2)$

$120.4(2)$

$120.0(2)$

118.1 (2)

120.1 (2)

$120.2(2)$

$119.8(2)$

$117.3(2)$

$120.3(2)$

119.7 (2)

$112.7(2)$

$120.3(2)$

$120.8(2)$

$124.1(2)$

$119.9(2)$

$121.9(2)$

$102.6(3)$
$\mathrm{N}(1)-\mathrm{Pd}(1)-\mathrm{Cl}(1)$

$\mathrm{N}(1)-\mathrm{Pd}(1)-\mathrm{Cl}(2)$

$\mathrm{Cl}(1)-\mathrm{Pd}(1)-\mathrm{Cl}$ (2)

$\mathrm{P}(1)-\mathrm{Pd}(1)-\mathrm{Br}(1)$

$\mathrm{Cl}$ (2) $-\mathrm{Pd}(1)-\mathrm{Br}(1)$

$\mathrm{P}(1)-\mathrm{Pd}(1)-\mathrm{Br}(2)$

$\mathrm{Cl}(2)-\mathrm{Pd}(1)-\mathrm{Br}(2)$

$\mathrm{C}(2)-\mathrm{P}(1)-\mathrm{C}(8)$

$C(8)-P(1)-C(1)$

$\mathrm{C}(8)-\mathrm{P}(1)-\mathrm{Pd}(1)$

$\mathrm{N}(1)-\mathrm{P}(2)-\mathrm{C}(20)$

$\mathrm{C}(20)-\mathrm{P}(2)-\mathrm{C}(14)$

$\mathrm{C}(20)-\mathrm{P}(2)-\mathrm{C}(1)$

$\mathrm{C}(30)-\mathrm{O}(1)-\mathrm{C}(33)$

$\mathrm{C}(26)-\mathrm{N}(1)-\mathrm{Pd}(1)$

$\mathrm{P}(2)-\mathrm{C}(1)-\mathrm{P}(1)$

$C(7)-C(2)-P(1)$

$C(4)-C(3)-C(2)$

$C(6)-C(5)-C(4)$

$\mathrm{C}(2)-\mathrm{C}(7)-\mathrm{C}(6)$

$C(13)-C(8)-P(1)$

$C(10)-C(9)-C(8)$

$\mathrm{C}(10)-\mathrm{C}(11)-\mathrm{C}(12)$

$C(8)-C(13)-C(12)$

$C(19)-C(14)-P(2)$

$C(16)-C(15)-C(14)$

$C(16)-C(17)-C(18)$

$C(18)-C(19)-C(14)$

$\mathrm{C}(21)-\mathrm{C}(20)-\mathrm{P}(2)$

$\mathrm{C}(20)-\mathrm{C}(21)-\mathrm{C}(22)$

$\mathrm{C}(22)-\mathrm{C}(23)-\mathrm{C}(24)$

$\mathrm{C}(24)-\mathrm{C}(25)-\mathrm{C}(20)$

$\mathrm{C}(32)-\mathrm{C}(27)-\mathrm{C}(28)$

$C(28)-C(27)-C(26)$

$\mathrm{C}(28)-\mathrm{C}(29)-\mathrm{C}(30)$

$\mathrm{O}(1)-\mathrm{C}(30)-\mathrm{C}(29)$

$\mathrm{C}(30)-\mathrm{C}(31)-\mathrm{C}(32)$

$\mathrm{C}(37)-\mathrm{O}(2)-\mathrm{C}(34)$

C $(36)-C(35)-C(34)$
$72.8(3)$

$98.1(1)$

$87.1(3)$

$88.5(1)$

$86.7(2)$

$176.90(6)$

$5.6(1)$

$107.5(1)$

$102.6(1)$

$117.80(7)$

$113.3(1)$

$108.4(1)$

$107.1(1)$

116.1 (2)

$123.8(1)$

$107.0(1)$

$119.5(2)$

$120.0(2)$

$120.3(2)$

$119.7(2)$

$122.7(2)$

$120.0(2)$

$120.0(2)$

$119.8(2)$

$121.9(2)$

$119.7(2)$

$120.3(2)$

$119.7(2)$

$122.8(2)$

$119.8(2)$

$120.5(2)$

$119.9(2)$

$118.2(2)$

$121.5(2)$

120.1 (2)

$115.9(2)$

$119.0(2)$

$105.1(2)$

$104.3(3)$ 
Table S1-4. Anisotropic displacement parameters ( $A^{\wedge} 2 \times 10^{\wedge} 3$ ) for

\begin{tabular}{|c|c|c|c|c|c|c|}
\hline atom & $\mathrm{U} 11$ & $\mathrm{U} 22$ & U33 & $\mathrm{U} 23$ & U13 & $\mathrm{U} 12$ \\
\hline $\mathrm{Pd}(1)$ & $19(1)$ & $19(1)$ & $24(1)$ & $5(1)$ & $6(1)$ & $4(1)$ \\
\hline $\operatorname{Br}(1)$ & $29(1)$ & $17(1)$ & $36(1)$ & $2(1)$ & $9(1)$ & $6(1)$ \\
\hline $\operatorname{Br}(2)$ & $27(1)$ & $20(1)$ & $20(1)$ & $2(1)$ & $8(1)$ & $2(1)$ \\
\hline $\mathrm{Cl}(2)$ & $27(1)$ & $20(1)$ & $20(1)$ & $2(1)$ & $8(1)$ & $2(1)$ \\
\hline $\mathrm{Cl}(1)$ & $29(1)$ & $17(1)$ & $36(1)$ & $2(1)$ & $9(1)$ & $6(1)$ \\
\hline P (1) & $20(1)$ & $18(1)$ & $22(1)$ & $3(1)$ & $6(1)$ & $5(1)$ \\
\hline$P(2)$ & $20(1)$ & $18(1)$ & $23(1)$ & $3(1)$ & $6(1)$ & $5(1)$ \\
\hline$O(1)$ & $30(1)$ & $34(1)$ & $38(1)$ & $14(1)$ & $7(1)$ & $14(1)$ \\
\hline$N(1)$ & $19(1)$ & $20(1)$ & $27(1)$ & $3(1)$ & $7(1)$ & $6(1)$ \\
\hline C (1) & $23(1)$ & $20(1)$ & $23(1)$ & $3(1)$ & $8(1)$ & $4(1)$ \\
\hline$C(2)$ & 27 (1) & $23(1)$ & 21 (1) & $4(1)$ & $6(1)$ & $11(1)$ \\
\hline$C(3)$ & $33(1)$ & $29(1)$ & $29(1)$ & $8(1)$ & $11(1)$ & $10(1)$ \\
\hline$C(4)$ & $49(2)$ & $35(1)$ & $30(1)$ & $13(1)$ & $15(1)$ & $19(1)$ \\
\hline$C(5)$ & $48(2)$ & $40(1)$ & $27(1)$ & $5(1)$ & $3(1)$ & $21(1)$ \\
\hline$C(6)$ & $35(1)$ & $41(1)$ & $32(1)$ & $3(1)$ & $-1(1)$ & $16(1)$ \\
\hline$C(7)$ & $26(1)$ & $30(1)$ & $29(1)$ & $5(1)$ & $4(1)$ & $8(1)$ \\
\hline$C(8)$ & 21 (1) & $18(1)$ & 31 (1) & $3(1)$ & $7(1)$ & $6(1)$ \\
\hline$C(9)$ & $30(1)$ & $26(1)$ & $33(1)$ & $5(1)$ & $6(1)$ & $12(1)$ \\
\hline$C(10)$ & $29(1)$ & $27(1)$ & $44(1)$ & $6(1)$ & $4(1)$ & $12(1)$ \\
\hline$C(11)$ & $29(1)$ & $26(1)$ & $58(2)$ & $10(1)$ & $17(1)$ & $13(1)$ \\
\hline$C(12)$ & $43(1)$ & $36(1)$ & $45(1)$ & $9(1)$ & $24(1)$ & $20(1)$ \\
\hline$C(13)$ & $33(1)$ & $32(1)$ & 31 (1) & $7(1)$ & $11(1)$ & $15(1)$ \\
\hline$C(14)$ & $23(1)$ & 21 (1) & $24(1)$ & $3(1)$ & $6(1)$ & $6(1)$ \\
\hline$C(15)$ & $25(1)$ & $24(1)$ & $27(1)$ & $4(1)$ & $8(1)$ & $5(1)$ \\
\hline$C(16)$ & $35(1)$ & $26(1)$ & $26(1)$ & $6(1)$ & $7(1)$ & $8(1)$ \\
\hline$C(17)$ & $33(1)$ & 32 (1) & $28(1)$ & $4(1)$ & $2(1)$ & $15(1)$ \\
\hline C (18) & $26(1)$ & $45(1)$ & $36(1)$ & $10(1)$ & $8(1)$ & $15(1)$ \\
\hline C (19) & $23(1)$ & $36(1)$ & 31 (1) & $9(1)$ & $7(1)$ & $9(1)$ \\
\hline$C(20)$ & $27(1)$ & $20(1)$ & 25 (1) & $4(1)$ & $10(1)$ & $6(1)$ \\
\hline$C(21)$ & 37 (1) & $24(1)$ & $23(1)$ & $2(1)$ & $6(1)$ & $6(1)$ \\
\hline$C(22)$ & $48(2)$ & $27(1)$ & $27(1)$ & $-3(1)$ & $5(1)$ & $2(1)$ \\
\hline C (23) & $55(2)$ & 21 (1) & $36(1)$ & $0(1)$ & $14(1)$ & $7(1)$ \\
\hline$C(24)$ & $45(1)$ & $25(1)$ & $39(1)$ & $7(1)$ & $14(1)$ & $14(1)$ \\
\hline$C(25)$ & 31 (1) & $23(1)$ & 31 (1) & $5(1)$ & $9(1)$ & $8(1)$ \\
\hline$C(26)$ & $24(1)$ & $28(1)$ & $25(1)$ & $5(1)$ & $9(1)$ & $9(1)$ \\
\hline C (27) & 22 (1) & $22(1)$ & $26(1)$ & $4(1)$ & $9(1)$ & $6(1)$ \\
\hline C (28) & $25(1)$ & $22(1)$ & $33(1)$ & $1(1)$ & $8(1)$ & $7(1)$ \\
\hline C (29) & $25(1)$ & $29(1)$ & $29(1)$ & $1(1)$ & $4(1)$ & $7(1)$ \\
\hline$C(30)$ & $22(1)$ & $28(1)$ & 32 (1) & $11(1)$ & $9(1)$ & $9(1)$ \\
\hline C (31) & $29(1)$ & $22(1)$ & $35(1)$ & $4(1)$ & $9(1)$ & $9(1)$ \\
\hline C (32) & $26(1)$ & $25(1)$ & 31 (1) & $-1(1)$ & $5(1)$ & $7(1)$ \\
\hline C (33) & $36(1)$ & $37(1)$ & $47(2)$ & $17(1)$ & $15(1)$ & $21(1)$ \\
\hline$O(2)$ & $47(1)$ & $59(1)$ & $51(1)$ & $9(1)$ & $9(1)$ & $20(1)$ \\
\hline$C(34)$ & $53(2)$ & $41(2)$ & $100(3)$ & $11(2)$ & $30(2)$ & $14(1)$ \\
\hline C (35) & $52(2)$ & $64(2)$ & $200(6)$ & $-52(3)$ & $-38(3)$ & $33(2)$ \\
\hline$C(36)$ & 77 (3) & $66(2)$ & 75 (3) & $25(2)$ & $34(2)$ & $37(2)$ \\
\hline C (37) & $40(2)$ & $56(2)$ & $63(2)$ & $-4(2)$ & $12(1)$ & $17(1)$ \\
\hline
\end{tabular}

The anisotropic displacement factor exponent takes the form $2 \mathrm{pi}^{\wedge} 2\left[\mathrm{~h}^{\wedge} 2 \mathrm{a}^{\star \wedge} 2 \mathrm{U}(11)+\ldots+2 \mathrm{hka} \mathrm{b}^{\star} \mathrm{U}(12)\right]$ 
Table S1-5. Hydrogen Coordinates (A x 10^4) and equivalent isotropic displacement parameters $\left(A^{\wedge} 2 \times 10^{\wedge} 3\right)$ for $5 a$

\begin{tabular}{|c|c|c|c|c|}
\hline atom & $\mathrm{x}$ & $\mathrm{Y}$ & z & $\mathrm{U}(\mathrm{eq})$ \\
\hline $\mathrm{H}(1 \mathrm{~A})$ & 1498 & 3721 & 2430 & 27 \\
\hline $\mathrm{H}(1 \mathrm{~B})$ & 161 & 4336 & 2108 & 27 \\
\hline $\mathrm{H}(3)$ & 32 & 3582 & 796 & 35 \\
\hline $\mathrm{H}(4)$ & -1249 & 3933 & -388 & 43 \\
\hline $\mathrm{H}(5)$ & -3823 & 2821 & -854 & 45 \\
\hline $\mathrm{H}(6)$ & -5125 & 1347 & -147 & 44 \\
\hline $\mathrm{H}(7)$ & -3850 & 978 & 1044 & 35 \\
\hline $\mathrm{H}(9)$ & 1184 & 899 & 2928 & 36 \\
\hline $\mathrm{H}(10)$ & 3157 & 69 & 2818 & 40 \\
\hline $\mathrm{H}(11)$ & 3682 & -217 & 1639 & 42 \\
\hline $\mathrm{H}(12)$ & 2168 & 228 & 557 & 45 \\
\hline $\mathrm{H}(13)$ & 200 & 1083 & 658 & 36 \\
\hline $\mathrm{H}(15)$ & -240 & 2122 & 4474 & 31 \\
\hline $\mathrm{H}(16)$ & 1303 & 1155 & 5289 & 36 \\
\hline $\mathrm{H}(17)$ & 3824 & 1579 & 5264 & 37 \\
\hline $\mathrm{H}(18)$ & 4844 & 3010 & 4449 & 41 \\
\hline $\mathrm{H}(19)$ & 3328 & 4005 & 3642 & 36 \\
\hline $\mathrm{H}(21)$ & 2538 & 5792 & 4511 & 35 \\
\hline $\mathrm{H}(22)$ & 3224 & 8111 & 4802 & 44 \\
\hline $\mathrm{H}(23)$ & 1801 & 9308 & 4123 & 46 \\
\hline $\mathrm{H}(24)$ & -333 & 8211 & 3150 & 42 \\
\hline $\mathrm{H}(25)$ & -998 & 5898 & 2831 & 34 \\
\hline $\mathrm{H}(26 \mathrm{~A})$ & -1758 & 4300 & 4138 & 30 \\
\hline $\mathrm{H}(26 \mathrm{~B})$ & -3024 & 2872 & 3959 & 30 \\
\hline $\mathrm{H}(28)$ & -4571 & 2689 & 2400 & 33 \\
\hline $\mathrm{H}(29)$ & -6290 & 3629 & 1710 & 34 \\
\hline $\mathrm{H}(31)$ & -4638 & 6915 & 3282 & 34 \\
\hline $\mathrm{H}(32)$ & -2951 & 5935 & 3975 & 33 \\
\hline $\mathrm{H}(33 \mathrm{~A})$ & -7034.9995 & 6925 & 2819 & 55 \\
\hline $\mathrm{H}(33 \mathrm{~B})$ & -7751 & 7199 & 1985 & 55 \\
\hline $\mathrm{H}(33 \mathrm{C})$ & -5966 & 7773 & 2337 & 55 \\
\hline $\mathrm{H}(34 \mathrm{~A})$ & -2972 & 5794 & 1653 & 75 \\
\hline $\mathrm{H}(34 \mathrm{~B})$ & -3028 & 4498 & 1121 & 75 \\
\hline $\mathrm{H}(35 \mathrm{~A})$ & -3396 & 5498 & 26 & 139 \\
\hline $\mathrm{H}(35 \mathrm{~B})$ & -4106 & 6357 & 514 & 139 \\
\hline $\mathrm{H}(36 \mathrm{~A})$ & -2193 & 8148 & 579 & 79 \\
\hline $\mathrm{H}(36 \mathrm{~B})$ & -1537 & 7309 & 49 & 79 \\
\hline $\mathrm{H}(37 \mathrm{~A})$ & 308 & 7572 & 1106 & 63 \\
\hline $\mathrm{H}(37 \mathrm{~B})$ & -722 & 7833 & 1652 & 63 \\
\hline
\end{tabular}


Figure S2: Molecular structure of 5c

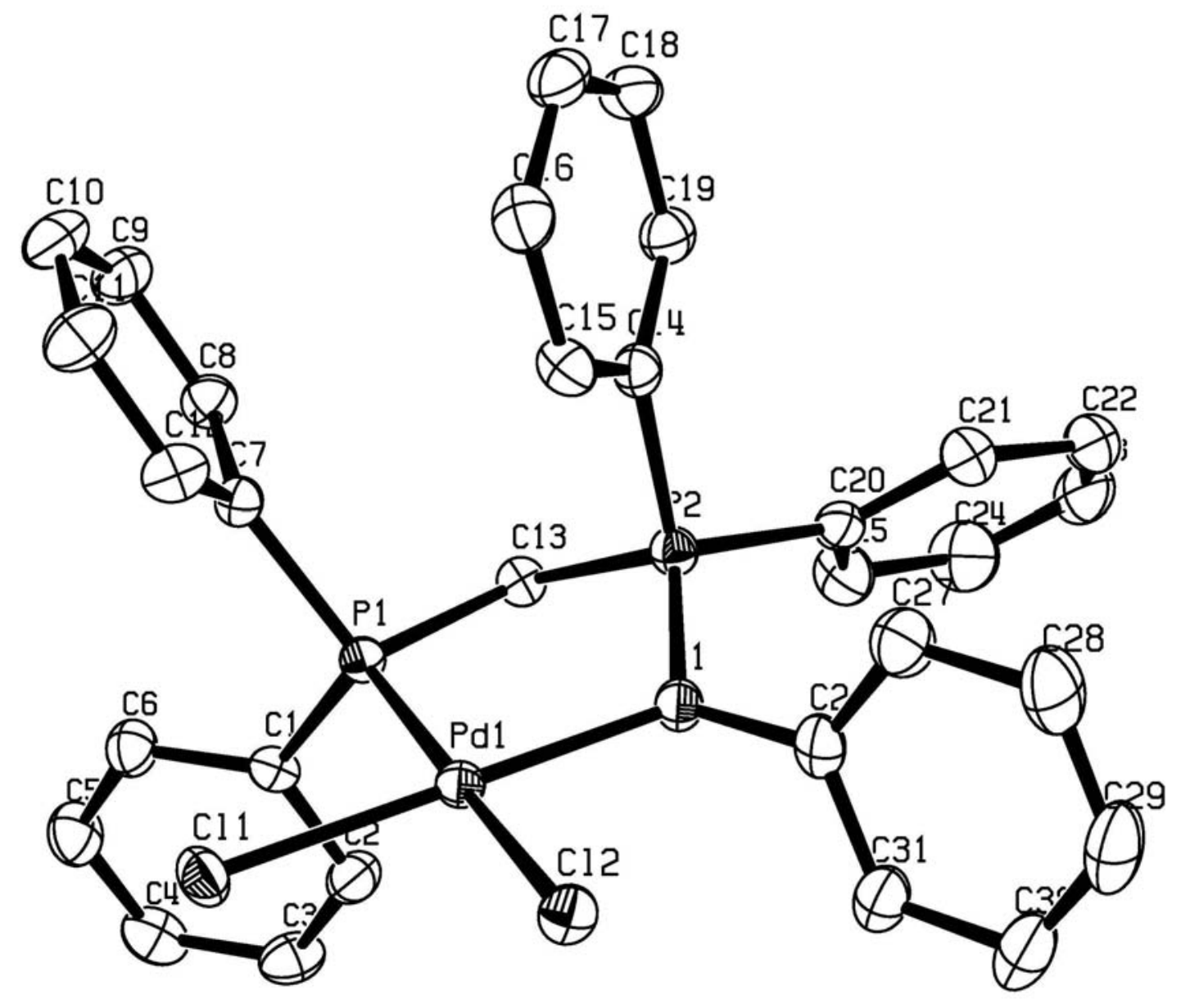


Table S2-1. Crystal data for $\mathbf{5 c}$

Compound

Molecular formula

Molecular weight

Crystal habit

Crystal dimensions(mm)

Crystal system

Space group

$\mathrm{a}(\AA)$

$\mathrm{b}(\AA)$

$\mathrm{c}(\AA)$

$\alpha\left(^{\circ}\right)$

$\beta\left(^{\circ}\right)$

$\gamma\left({ }^{\circ}\right)$

$\mathrm{V}\left(\AA^{3}\right)$

$\mathrm{Z}$

$\mathrm{d}\left(\mathrm{g}-\mathrm{cm}^{-3}\right)$

$\mathrm{F}(000)$

$\mu\left(\mathrm{cm}^{-1}\right)$

Absorption corrections

Diffractometer

$\mathrm{X}$-ray source

$\lambda(\AA)$

Monochromator

$\mathrm{T}(\mathrm{K})$

Scan mode

Maximum $\theta$

HKL ranges

Reflections measured

Unique data

Rint

Reflections used

Criterion

Refinement type

Hydrogen atoms

Parameters refined

Reflections / parameter

wR2

$\mathrm{R} 1$

Weights $a, b$

GoF

difference peak / hole (e $\left.\AA^{-3}\right)$
$5 c$

$\mathrm{C}_{31} \mathrm{H}_{27} \mathrm{Cl}_{2} \mathrm{NP}_{2} \mathrm{Pd}$

652.78

orange needle

$0.22 \times 0.16 \times 0.14$

monoclinic

$\mathrm{P} 2{ }_{1} / \mathrm{c}$

$11.3600(10)$

$14.8220(10)$

$17.0950(10)$

90.00

$105.1700(10)$

90.00

2778.1(3)

4

1.561

1320

0.998

multi-scan ; 0.8104 min, 0.8730 max

KappaCCD

$\mathrm{MoK} \alpha$

0.71069

graphite

150.0(1)

phi and omega scans

29.99

$-1515 ;-1920 ;-2424$

14300

8054

0.0224

6288

I $>2 \sigma \mathrm{I})$

Fsqd

mixed

334

18

0.0939

0.0341

$0.0484 ; 0.0000$

1.019

$1.096(0.092) /-1.123(0.092)$ 
Table S2-2. Atomic Coordinates (A x 10^4) and equivalent isotropic displacement parameters $\left(A^{\wedge} 2 \times 10^{\wedge} 3\right)$ for $5 \mathbf{c}$

\begin{tabular}{|c|c|c|c|c|}
\hline atom & $\mathrm{x}$ & y & z & $\mathrm{U}$ (eq) \\
\hline $\mathrm{Pd}(1)$ & $1249(1)$ & $3062(1)$ & $2450(1)$ & $17(1)$ \\
\hline $\mathrm{Cl}(1)$ & $-755(1)$ & $3042(1)$ & $1698(1)$ & $25(1)$ \\
\hline $\mathrm{Cl}(2)$ & $1955(1)$ & $2919(1)$ & $1270(1)$ & $21(1)$ \\
\hline$P(1)$ & $554(1)$ & $3061(1)$ & $3544(1)$ & $17(1)$ \\
\hline$P(2)$ & $3189(1)$ & $2756(1)$ & $4105(1)$ & $19(1)$ \\
\hline $\mathrm{N}(1)$ & $3011(2)$ & $3144(1)$ & $3196(1)$ & $20(1)$ \\
\hline$C(1)$ & $-421(2)$ & 3991 (1) & $3664(1)$ & $20(1)$ \\
\hline$C(2)$ & $25(2)$ & $4873(1)$ & $3672(1)$ & $28(1)$ \\
\hline$C(3)$ & $-702(2)$ & $5604(2)$ & $3728(1)$ & $32(1)$ \\
\hline$C(4)$ & $-1885(2)$ & $5460(2)$ & $3775(1)$ & $32(1)$ \\
\hline$C(5)$ & $-2334(2)$ & $4600(2)$ & $3777(2)$ & $33(1)$ \\
\hline$C(6)$ & $-1606(2)$ & $3857(2)$ & $3717(1)$ & $27(1)$ \\
\hline$C(7)$ & $-172(2)$ & $2024(1)$ & $3718(1)$ & $20(1)$ \\
\hline$C(8)$ & $-365(2)$ & $1852(1)$ & $4482(1)$ & $25(1)$ \\
\hline$C(9)$ & $-858(2)$ & $1037(2)$ & $4633(2)$ & 31 (1) \\
\hline$C(10)$ & $-1170(2)$ & $389(2)$ & $4031(2)$ & $33(1)$ \\
\hline$C(11)$ & $-994(2)$ & $556(2)$ & $3274(2)$ & $34(1)$ \\
\hline$C(12)$ & $-493(2)$ & $1373(1)$ & $3116(1)$ & $27(1)$ \\
\hline$C(13)$ & $1900(2)$ & $3192(1)$ & $4413(1)$ & $20(1)$ \\
\hline$C(14)$ & $3147(2)$ & $1544(1)$ & $4195(1)$ & $21(1)$ \\
\hline$C(15)$ & $2615(2)$ & $1033(1)$ & $3512(1)$ & $25(1)$ \\
\hline$C(16)$ & $2498(2)$ & $112(2)$ & $3580(1)$ & $29(1)$ \\
\hline$C(17)$ & $2940(2)$ & $-306(1)$ & $4319(2)$ & $29(1)$ \\
\hline$C(18)$ & $3472(2)$ & $200(2)$ & $4998(1)$ & $29(1)$ \\
\hline C (19) & $3574(2)$ & $1130(1)$ & $4947(1)$ & $25(1)$ \\
\hline$C(20)$ & $4582(2)$ & $3129(1)$ & $4795(1)$ & $23(1)$ \\
\hline$C(21)$ & $5679(2)$ & $2675(2)$ & $4825(2)$ & $30(1)$ \\
\hline$C(22)$ & $6759(2)$ & $2980(2)$ & $5333(2)$ & $37(1)$ \\
\hline C (23) & $6766(2)$ & $3710(2)$ & $5826(2)$ & $44(1)$ \\
\hline$C(24)$ & $5702(3)$ & $4160(2)$ & $5806(2)$ & $44(1)$ \\
\hline$C(25)$ & $4609(2)$ & $3877(2)$ & $5292(1)$ & $33(1)$ \\
\hline$C(26)$ & $4016(2)$ & $3158(2)$ & $2844(1)$ & $24(1)$ \\
\hline C (27) & $4726(2)$ & $2410(2)$ & $2806(2)$ & $33(1)$ \\
\hline$C(28)$ & $5700(2)$ & $2461(2)$ & $2456(2)$ & $40(1)$ \\
\hline$C(29)$ & $5951(2)$ & $3257(2)$ & $2126(2)$ & $41(1)$ \\
\hline C (30) & $5242(2)$ & $4009(2)$ & $2142(2)$ & $37(1)$ \\
\hline$C(31)$ & $4283(2)$ & $3968(2)$ & $2503(1)$ & $29(1)$ \\
\hline
\end{tabular}

$\mathrm{U}(\mathrm{eq})$ is defined as $1 / 3$ the trace of the Uij tensor. 
Table S2-3. Bond lengths (A) and angles (deg) for 5c

$$
\begin{aligned}
& \mathrm{Pd}(1)-\mathrm{N}(1) \\
& \mathrm{Pd}(1)-\mathrm{C}(1) \\
& \mathrm{P}(1)-\mathrm{C}(7) \\
& \mathrm{P}(1)-\mathrm{C}(13) \\
& \mathrm{P}(2)-\mathrm{C}(20) \\
& \mathrm{P}(2)-\mathrm{C}(14) \\
& \mathrm{C}(1)-\mathrm{C}(6) \\
& \mathrm{C}(2)-\mathrm{C}(3) \\
& \mathrm{C}(4)-\mathrm{C}(5) \\
& \mathrm{C}(7)-\mathrm{C}(12) \\
& \mathrm{C}(8)-\mathrm{C}(9) \\
& \mathrm{C}(10)-\mathrm{C}(11) \\
& \mathrm{C}(14)-\mathrm{C}(15) \\
& \mathrm{C}(15)-\mathrm{C}(16) \\
& \mathrm{C}(17)-\mathrm{C}(18) \\
& \mathrm{C}(20)-\mathrm{C}(25) \\
& \mathrm{C}(21)-\mathrm{C}(22) \\
& \mathrm{C}(23)-\mathrm{C}(24) \\
& \mathrm{C}(26)-\mathrm{C}(27) \\
& \mathrm{C}(27)-\mathrm{C}(28) \\
& C(29)-\mathrm{C}(30)
\end{aligned}
$$

$\mathrm{N}(1)-\mathrm{Pd}(1)-\mathrm{P}(1)$

$\mathrm{P}(1)-\mathrm{Pd}(1)-\mathrm{Cl}(1)$

$\mathrm{P}(1)-\mathrm{Pd}(1)-\mathrm{Cl}(2)$

$C(7)-P(1)-C(1)$

$C(1)-P(1)-C(13)$

$\mathrm{C}(1)-\mathrm{P}(1)-\mathrm{Pd}(1)$

$\mathrm{N}(1)-\mathrm{P}(2)-\mathrm{C}(20)$

$\mathrm{C}(20)-\mathrm{P}(2)-\mathrm{C}(13)$

$\mathrm{C}(20)-\mathrm{P}(2)-\mathrm{C}(14)$

$\mathrm{C}(26)-\mathrm{N}(1)-\mathrm{P}(2)$

$\mathrm{P}(2)-\mathrm{N}(1)-\mathrm{Pd}(1)$

$C(6)-C(1)-P(1)$

$C(3)-C(2)-C(1)$

$C(5)-C(4)-C(3)$

$C(1)-C(6)-C(5)$

$\mathrm{C}(12)-\mathrm{C}(7)-\mathrm{P}(1)$

$C(9)-C(8)-C(7)$

$C(11)-C(10)-C(9)$

$C(7)-C(12)-C(11)$

$\mathrm{C}(15)-\mathrm{C}(14)-\mathrm{C}(19)$

$\mathrm{C}(19)-\mathrm{C}(14)-\mathrm{P}(2)$

$\mathrm{C}(17)-\mathrm{C}(16)-\mathrm{C}(15)$

$C(17)-C(18)-C(19)$

$C(25)-C(20)-C(21)$

$\mathrm{C}(21)-\mathrm{C}(20)-\mathrm{P}(2)$

C (23) $-\mathrm{C}(22)-\mathrm{C}(21)$

$C(23)-C(24)-C(25)$

$C(27)-C(26)-C(31)$

$\mathrm{C}(31)-\mathrm{C}(26)-\mathrm{N}(1)$

C (29) $-\mathrm{C}(28)-\mathrm{C}(27)$

C (29) $-C(30)-C(31)$
$2.076(2)$
$2.3035(6$
$1.805(2)$
$1.842(2)$
$1.797(2)$
$1.804(2)$
$1.387(3)$
$1.380(3)$
$1.374(3)$
$1.388(3)$
$1.383(3)$
$1.382(3)$
$1.390(3)$
$1.380(3)$
$1.382(3)$
$1.392(3)$
$1.381(3)$
$1.373(4)$
$1.384(3)$
$1.391(3)$
$1.381(4)$

$88.90(5)$

$87.25(2)$

$174.71(2)$

$108.1(1)$

$103.4(1)$

$117.27(7)$

$112.8(1)$

$109.9(1)$

$106.8(1)$

$119.9(2)$

$115.2(1)$

$122.1(2)$

$120.7(2)$

$120.7(2)$

$119.7(2)$

$120.8(2)$

$120.1(2)$

$120.0(2)$

$120.1(2)$

$120.4(2)$

$120.5(2)$

$120.1(2)$

$120.7(2)$

$118.8(2)$

$119.8(2)$

$120.4(3)$

$120.4(2)$

$118.3(2)$

$118.3(2)$

$120.0(3)$

$120.2(2)$
$\mathrm{Pd}(1)-\mathrm{P}(1)$

$\mathrm{Pd}(1)-\mathrm{Cl}(2)$

$\mathrm{P}(1)-\mathrm{C}(1)$

$\mathrm{P}(2)-\mathrm{N}(1)$

$P(2)-C(13)$

$\mathrm{N}(1)-\mathrm{C}(26)$

$C(1)-C(2)$

$C(3)-C(4)$

$C(5)-C(6)$

$C(7)-C(8)$

$C(9)-C(10)$

C (11) -C (12)

C (14) $-\mathrm{C}(19)$

C (16) $-\mathrm{C}(17)$

C (18) $-\mathrm{C}(19)$

C (20)-C (21)

C (22) $-\mathrm{C}(23)$

C (24)-C (25)

$C(26)-C(31)$

C (28)-C (29)

C (30) $-\mathrm{C}(31)$

$\begin{array}{ll}\mathrm{N}(1)-\mathrm{Pd}(1)-\mathrm{C} 1(1) & 175.35(5) \\ \mathrm{N}(1)-\mathrm{Pd}(1)-\mathrm{C}(12) & 92.39(5) \\ \mathrm{C} 1(1)-\mathrm{Pd}(1)-\mathrm{Cl}(2) & 91.67(2) \\ \mathrm{C}(7)-\mathrm{P}(1)-\mathrm{C}(13) & 106.1(1) \\ \mathrm{C}(7)-\mathrm{P}(1)-\mathrm{Pd}(1) & 114.75(7) \\ \mathrm{C}(13)-\mathrm{P}(1)-\mathrm{Pd}(1) & 106.03(7) \\ \mathrm{N}(1)-\mathrm{P}(2)-\mathrm{C}(13) & 104.2(1) \\ \mathrm{N}(1)-\mathrm{P}(2)-\mathrm{C}(14) & 115.9(1) \\ \mathrm{C}(13)-\mathrm{P}(2)-\mathrm{C}(14) & 106.9(1) \\ \mathrm{C}(26)-\mathrm{N}(1)-\mathrm{Pd}(1) & 119.5(1) \\ \mathrm{C}(6)-\mathrm{C}(1)-\mathrm{C}(2) & 119.2(2) \\ \mathrm{C}(2)-\mathrm{C}(1)-\mathrm{P}(1) & 118.7(2) \\ \mathrm{C}(2)-\mathrm{C}(3)-\mathrm{C}(4) & 119.5(2) \\ \mathrm{C}(4)-\mathrm{C}(5)-\mathrm{C}(6) & 120.2(2) \\ \mathrm{C}(12)-\mathrm{C}(7)-\mathrm{C}(8) & 119.3(2) \\ \mathrm{C}(8)-\mathrm{C}(7)-\mathrm{P}(1) & 119.8(2) \\ \mathrm{C}(8)-\mathrm{C}(9)-\mathrm{C}(10) & 120.4(2) \\ \mathrm{C}(10)-\mathrm{C}(11)-\mathrm{C}(12) & 120.2(2) \\ \mathrm{P}(2)-\mathrm{C}(13)-\mathrm{P}(1) & 107.1(1) \\ \mathrm{C}(15)-\mathrm{C}(14)-\mathrm{P}(2) & 119.0(2) \\ \mathrm{C}(16)-\mathrm{C}(15)-\mathrm{C}(14) & 119.9(2) \\ \mathrm{C}(16)-\mathrm{C}(17)-\mathrm{C}(18) & 120.0(2) \\ \mathrm{C}(18)-\mathrm{C}(19)-\mathrm{C}(14) & 118.8(2) \\ \mathrm{C}(25)-\mathrm{C}(20)-\mathrm{P}(2) & 121.5(2) \\ \mathrm{C}(22)-\mathrm{C}(21)-\mathrm{C}(20) & 120.0(2) \\ \mathrm{C}(22)-\mathrm{C}(23)-\mathrm{C}(24) & 120.3(2) \\ \mathrm{C}(24)-\mathrm{C}(25)-\mathrm{C}(20) & 120.1(2) \\ \mathrm{C}(27)-\mathrm{C}(26)-\mathrm{N}(1) & 123.4(2) \\ \mathrm{C}(26)-\mathrm{C}(27)-\mathrm{C}(28) & 121.1(2) \\ \mathrm{C}(28)-\mathrm{C}(29)-\mathrm{C}(30) & 120.1(2) \\ \mathrm{C}(30)-\mathrm{C}(31)-\mathrm{C}(26) & 120.3(2) \\ & \end{array}$

$2.2135(6)$
$2.3668(5)$
$1.813(2)$
$1.619(2)$
$1.800(2)$
$1.422(3)$
$1.402(3)$
$1.383(3)$
$1.396(3)$
$1.404(3)$
$1.384(3)$
$1.395(3)$
$1.391(3)$
$1.379(3)$
$1.388(3)$
$1.406(3)$
$1.371(4)$
$1.384(3)$
$1.401(3)$
$1.369(4)$
$1.387(3)$

$175.35(5)$

$92.39(5)$

$7(2)$

$114.75(7)$

$106.03(7)$

$106.9(1)$

$119.5(1)$

(2)

$119.5(2)$

$120.2(2)$

$120.2(2)$

$119.0(2)$

$9(2)$

$121.5(2)$

$20.0(2)$

$123.4(2)$

$120.1(2)$ 
Table S2-4. Anisotropic displacement parameters ( $A^{\wedge} 2 \times 10^{\wedge} 3$ ) for $\mathbf{5 c}$

\begin{tabular}{|c|c|c|c|c|c|c|}
\hline atom & U11 & $\mathrm{U} 22$ & U33 & U23 & U13 & $\mathrm{U} 12$ \\
\hline $\mathrm{Pd}(1)$ & $18(1)$ & $18(1)$ & $15(1)$ & $1(1)$ & $4(1)$ & $0(1)$ \\
\hline $\mathrm{Cl}(1)$ & $19(1)$ & $32(1)$ & 21 (1) & $2(1)$ & $0(1)$ & $1(1)$ \\
\hline $\mathrm{Cl}(2)$ & $24(1)$ & $27(1)$ & $14(1)$ & $-2(1)$ & $6(1)$ & $-2(1)$ \\
\hline $\mathrm{P}(1)$ & $17(1)$ & $18(1)$ & $16(1)$ & $0(1)$ & $5(1)$ & $0(1)$ \\
\hline $\mathrm{P}(2)$ & $18(1)$ & $21(1)$ & $17(1)$ & $0(1)$ & $4(1)$ & $0(1)$ \\
\hline $\mathrm{N}(1)$ & $18(1)$ & $26(1)$ & $17(1)$ & $1(1)$ & $4(1)$ & $-1(1)$ \\
\hline$C(1)$ & $22(1)$ & $20(1)$ & $18(1)$ & $1(1)$ & $5(1)$ & $3(1)$ \\
\hline$C(2)$ & $28(1)$ & $23(1)$ & $34(1)$ & $2(1)$ & $13(1)$ & $-1(1)$ \\
\hline$C(3)$ & $42(2)$ & $20(1)$ & $36(1)$ & $0(1)$ & 15 (1) & $1(1)$ \\
\hline$C(4)$ & 38 (1) & 32 (1) & $29(1)$ & $-2(1)$ & 11 (1) & $13(1)$ \\
\hline$C(5)$ & 25 (1) & $34(1)$ & 41 (1) & $-2(1)$ & 12 (1) & $5(1)$ \\
\hline$C(6)$ & $23(1)$ & $25(1)$ & $33(1)$ & $-2(1)$ & $9(1)$ & $0(1)$ \\
\hline$C(7)$ & $19(1)$ & $20(1)$ & $22(1)$ & $1(1)$ & $6(1)$ & $0(1)$ \\
\hline$C(8)$ & $27(1)$ & $26(1)$ & $22(1)$ & $0(1)$ & $6(1)$ & $-1(1)$ \\
\hline$C(9)$ & $32(1)$ & 31 (1) & 31 (1) & $5(1)$ & $13(1)$ & $-4(1)$ \\
\hline C (10) & 37 (1) & $23(1)$ & $40(1)$ & $2(1)$ & $14(1)$ & $-7(1)$ \\
\hline C (11) & $42(2)$ & $25(1)$ & $37(1)$ & $-5(1)$ & $14(1)$ & $-7(1)$ \\
\hline $\mathrm{C}(12)$ & $34(1)$ & $24(1)$ & $26(1)$ & $-1(1)$ & $12(1)$ & $-1(1)$ \\
\hline C (13) & 21 (1) & $20(1)$ & $19(1)$ & $-2(1)$ & $5(1)$ & $1(1)$ \\
\hline$C(14)$ & $18(1)$ & $22(1)$ & $24(1)$ & $0(1)$ & $7(1)$ & $0(1)$ \\
\hline$C(15)$ & $24(1)$ & $24(1)$ & $25(1)$ & $0(1)$ & $4(1)$ & $4(1)$ \\
\hline$C(16)$ & $27(1)$ & $25(1)$ & $33(1)$ & $-8(1)$ & $6(1)$ & $-1(1)$ \\
\hline C (17) & $30(1)$ & $20(1)$ & $41(1)$ & $0(1)$ & $16(1)$ & $0(1)$ \\
\hline C (18) & 31 (1) & $27(1)$ & 31 (1) & $8(1)$ & 11 (1) & $2(1)$ \\
\hline C (19) & 25 (1) & $25(1)$ & $27(1)$ & $0(1)$ & $10(1)$ & $1(1)$ \\
\hline C (20) & 22 (1) & $25(1)$ & $20(1)$ & $4(1)$ & $3(1)$ & $-3(1)$ \\
\hline$C(21)$ & $24(1)$ & $32(1)$ & $32(1)$ & $4(1)$ & $4(1)$ & $1(1)$ \\
\hline $\mathrm{C}(22)$ & $23(1)$ & $42(2)$ & $40(2)$ & $12(1)$ & $-2(1)$ & $-4(1)$ \\
\hline$C(23)$ & $30(1)$ & $51(2)$ & $39(2)$ & $13(1)$ & $-8(1)$ & $-17(1)$ \\
\hline$C(24)$ & $44(2)$ & $41(2)$ & $38(2)$ & $-9(1)$ & $-3(1)$ & $-11(1)$ \\
\hline C (25) & 32 (1) & $32(1)$ & $32(1)$ & $-5(1)$ & $2(1)$ & $-2(1)$ \\
\hline C (26) & $19(1)$ & $37(1)$ & $14(1)$ & $-3(1)$ & $2(1)$ & $-3(1)$ \\
\hline C (27) & 31 (1) & $39(1)$ & 32 (1) & $3(1)$ & $14(1)$ & $4(1)$ \\
\hline C (28) & $29(1)$ & $57(2)$ & $38(2)$ & $-4(1)$ & 15 (1) & $6(1)$ \\
\hline C (29) & $28(1)$ & $65(2)$ & $36(2)$ & $-6(1)$ & $16(1)$ & $-9(1)$ \\
\hline$C(30)$ & 32 (1) & $52(2)$ & 31 (1) & $-1(1)$ & 12 (1) & $-16(1)$ \\
\hline C (31) & $25(1)$ & $37(1)$ & $24(1)$ & $-1(1)$ & $8(1)$ & $-6(1)$ \\
\hline
\end{tabular}

The anisotropic displacement factor exponent takes the form $2 \mathrm{pi}^{\wedge} 2\left[\mathrm{~h}^{\wedge} 2 \mathrm{a}^{\star \wedge} 2 \mathrm{U}(11)+\ldots+2 \mathrm{hka} \mathrm{b}^{\star} \mathrm{U}(12)\right]$ 
Table S2-5. Hydrogen Coordinates (A x 10^4) and equivalent isotropic displacement parameters $\left(A^{\wedge} 2 \times 10^{\wedge} 3\right)$ for $5 \mathbf{c}$

\begin{tabular}{|c|c|c|c|c|}
\hline atom & $\mathrm{x}$ & $\mathrm{Y}$ & $\mathrm{z}$ & $\mathrm{U}(\mathrm{eq})$ \\
\hline $\mathrm{H}(2)$ & 837 & 4970 & 3638 & 33 \\
\hline $\mathrm{H}(3)$ & -393.0000 & 6200 & 3734 & 38 \\
\hline $\mathrm{H}(4)$ & -2392 & 5960 & 3806 & 39 \\
\hline $\mathrm{H}(5)$ & -3143 & 4509 & 3820 & 40 \\
\hline $\mathrm{H}(6)$ & -1921 & 3263 & 3712 & 32 \\
\hline $\mathrm{H}(8)$ & -157 & 2295 & 4897 & 30 \\
\hline $\mathrm{H}(9)$ & -983 & 922 & 5151 & 37 \\
\hline $\mathrm{H}(10)$ & -1505 & -170.0000 & 4138 & 39 \\
\hline $\mathrm{H}(11)$ & -1216 & 111 & 2860 & 40 \\
\hline $\mathrm{H}(12)$ & -370 & 1483 & 2595 & 33 \\
\hline $\mathrm{H}(13 \mathrm{~A})$ & 2029 & 3837 & 4564 & 24 \\
\hline $\mathrm{H}(13 \mathrm{~B})$ & 1787 & 2852 & 4887 & 24 \\
\hline $\mathrm{H}(15)$ & 2333 & 1318 & 2999 & 30 \\
\hline $\mathrm{H}(16)$ & 2113 & -235.0000 & 3116 & 34 \\
\hline $\mathrm{H}(17)$ & 2878 & -942 & 4362 & 35 \\
\hline $\mathrm{H}(18)$ & 3771 & -91 & 5506 & 35 \\
\hline $\mathrm{H}(19)$ & 3930 & 1478 & 5416 & 30 \\
\hline $\mathrm{H}(21)$ & 5677 & 2158 & 4496 & 36 \\
\hline $\mathrm{H}(22)$ & 7501 & 2681 & 5340 & 44 \\
\hline $\mathrm{H}(23)$ & 7511 & 3906 & 6184 & 52 \\
\hline $\mathrm{H}(24)$ & 5715 & 4669 & 6146 & 52 \\
\hline $\mathrm{H}(25)$ & 3877 & 4194 & 5279 & 39 \\
\hline $\mathrm{H}(27)$ & 4547 & 1852 & 3023 & 39 \\
\hline $\mathrm{H}(28)$ & 6190 & 1944 & 2445 & 48 \\
\hline $\mathrm{H}(29)$ & 6615 & 3291 & 1885 & 49 \\
\hline $\mathrm{H}(30)$ & 5411 & 4558 & 1904 & 45 \\
\hline $\mathrm{H}(31)$ & 3805 & 4490 & 2520 & 34 \\
\hline
\end{tabular}


Figure S3: Molecular structure of 5e

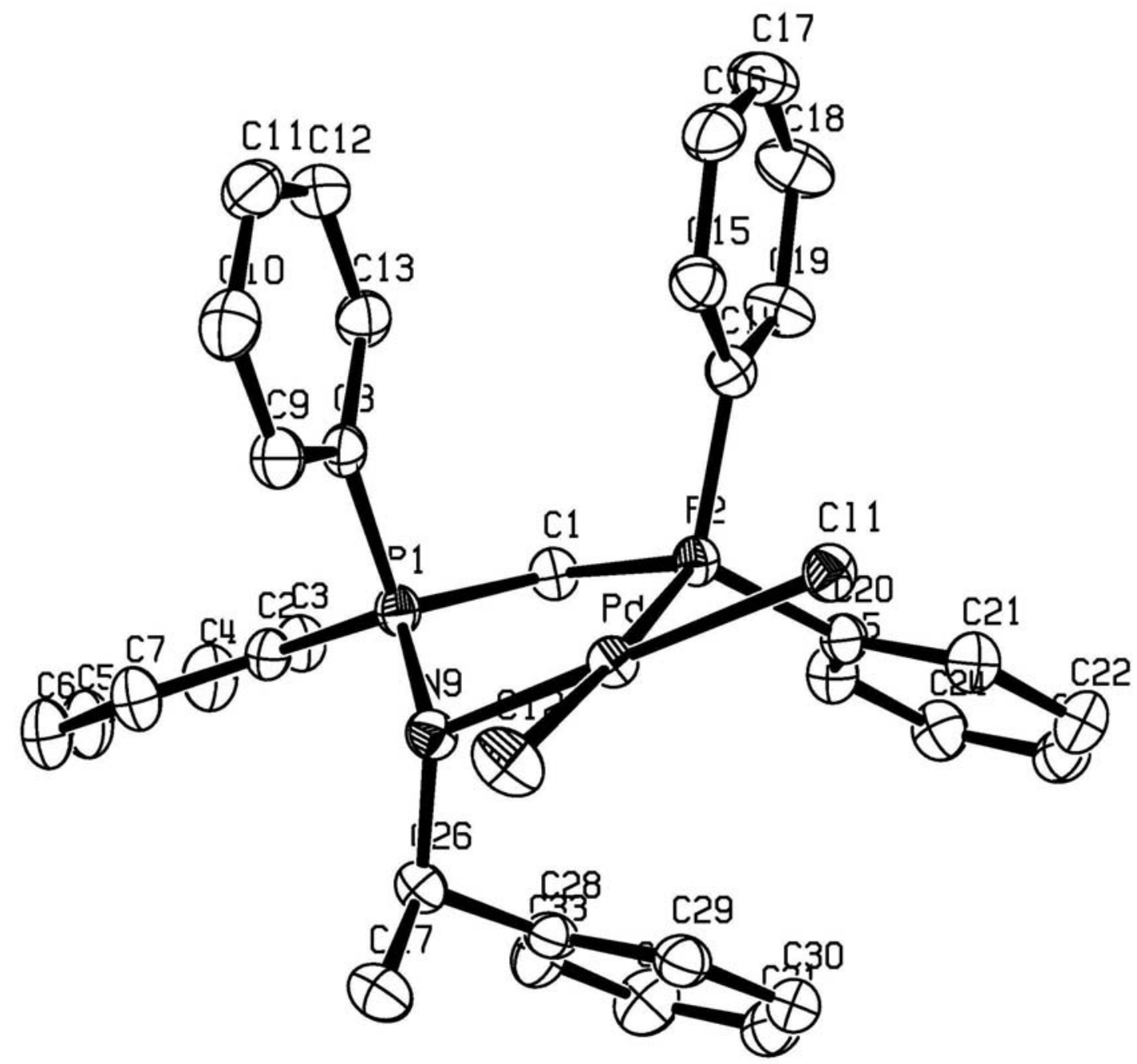


Table S3-1. Crystal data for $\mathbf{5 e}$

\begin{tabular}{|c|c|}
\hline Compound & $5 e$ \\
\hline Molecular formula & $\mathrm{C}_{33} \mathrm{H}_{31} \mathrm{Cl}_{2} \mathrm{NP}_{2} \mathrm{Pd}$ \\
\hline Molecular weight & 680.83 \\
\hline Crystal habit & orange plate \\
\hline Crystal dimensions $(\mathrm{mm})$ & $0.20 \times 0.16 \times 0.05$ \\
\hline Crystal system & orthorhombic \\
\hline Space group & $\mathrm{P} 2{ }_{1} 2_{1} 2_{1}$ \\
\hline $\mathrm{a}(\AA)$ & $12.3350(10)$ \\
\hline $\mathrm{b}(\AA)$ & $13.6480(10)$ \\
\hline$c(\AA)$ & $17.6430(10)$ \\
\hline$\alpha\left(^{\circ}\right)$ & 90.00 \\
\hline$\beta\left(\left(^{\circ}\right)\right.$ & 90.00 \\
\hline$\gamma\left({ }^{\circ}\right)$ & 90.00 \\
\hline $\mathrm{V}\left(\AA^{3}\right)$ & $2970.2(4)$ \\
\hline Z & 4 \\
\hline$d\left(g-\mathrm{cm}^{-3}\right)$ & 1.523 \\
\hline $\mathrm{F}(000)$ & 1384 \\
\hline$\mu\left(\mathrm{cm}^{-1}\right)$ & 0.937 \\
\hline Absorption corrections & multi-scan ; $0.8348 \min , 0.9547 \max$ \\
\hline Diffractometer & KappaCCD \\
\hline X-ray source & $\operatorname{MoK} \alpha$ \\
\hline$\lambda(\AA)$ & 0.71069 \\
\hline Monochromator & graphite \\
\hline $\mathrm{T}(\mathrm{K})$ & $150.0(1)$ \\
\hline Scan mode & phi and omega scans \\
\hline Maximum $\theta$ & 30.02 \\
\hline HKL ranges & $-1717 ;-1919 ;-2424$ \\
\hline Reflections measured & 8575 \\
\hline Unique data & 8575 \\
\hline Rint & 0.0000 \\
\hline Reflections used & 7398 \\
\hline Criterion & $\mathrm{I}>2 \sigma \mathrm{I})$ \\
\hline Refinement type & Fsqd \\
\hline Hydrogen atoms & mixed \\
\hline Parameters refined & 354 \\
\hline Reflections / parameter & 20 \\
\hline wR2 & 0.0819 \\
\hline $\mathrm{R} 1$ & 0.0337 \\
\hline Flack's parameter & $-0.022(19)$ \\
\hline Weights $a, b$ & $0.0427 ; 0.0000$ \\
\hline GoF & 1.008 \\
\hline difference peak / hole $\left(\mathrm{e} \AA^{-3}\right)$ & $0.857(0.087) /-0.923(0.087)$ \\
\hline
\end{tabular}


Table S3-2. Atomic Coordinates (A x 10^4) and equivalent isotropic displacement parameters $\left(A^{\wedge} 2 \times 10^{\wedge} 3\right)$ for 5

\begin{tabular}{|c|c|c|c|c|}
\hline atom & $\mathrm{x}$ & $\mathrm{y}$ & z & $\mathrm{U}(\mathrm{eq})$ \\
\hline $\mathrm{Pd}(1)$ & $3166(1)$ & $32(1)$ & $2246(1)$ & $20(1)$ \\
\hline $\mathrm{Cl}(1)$ & $4993(1)$ & $-17(1)$ & $1919(1)$ & $24(1)$ \\
\hline $\mathrm{Cl}(2)$ & $2680(1)$ & $-155(1)$ & $956(1)$ & 31 (1) \\
\hline $\mathrm{P}(1)$ & $1443(1)$ & $-558(1)$ & $3333(1)$ & 21 (1) \\
\hline$P(2)$ & $3648(1)$ & $89(1)$ & $3471(1)$ & $20(1)$ \\
\hline$N(9)$ & $1535(2)$ & $77(2)$ & $2573(1)$ & $22(1)$ \\
\hline$C(1)$ & $2397(2)$ & $-82(2)$ & $4017(1)$ & $23(1)$ \\
\hline$C(2)$ & $122(2)$ & $-537(2)$ & $3773(2)$ & $23(1)$ \\
\hline$C(3)$ & $23(2)$ & $-263(2)$ & $4533(2)$ & $28(1)$ \\
\hline$C(4)$ & $-995(2)$ & $-220(2)$ & $4868(2)$ & $34(1)$ \\
\hline$C(5)$ & $-1909(3)$ & $-443(2)$ & $4448(2)$ & $36(1)$ \\
\hline$C(6)$ & $-1806(3)$ & $-740(2)$ & $3704(2)$ & $36(1)$ \\
\hline$C(7)$ & $-799(2)$ & $-793(2)$ & $3366(2)$ & $30(1)$ \\
\hline$C(8)$ & $1810(2)$ & $-1802(2)$ & $3116(2)$ & $22(1)$ \\
\hline$C(9)$ & $1673(2)$ & $-2148(2)$ & $2381(2)$ & 27 (1) \\
\hline$C(10)$ & $1945(3)$ & $-3114(2)$ & $2211(2)$ & $33(1)$ \\
\hline$C(11)$ & $2351(2)$ & $-3722(2)$ & $2767(2)$ & $35(1)$ \\
\hline C (12) & $2483(3)$ & $-3376(2)$ & $3498(2)$ & $35(1)$ \\
\hline$C(13)$ & $2213(2)$ & $-2419(2)$ & $3679(2)$ & $28(1)$ \\
\hline$C(14)$ & $4494(2)$ & $-914(2)$ & $3793(2)$ & $23(1)$ \\
\hline$C(15)$ & $4735(2)$ & -1701 (2) & $3320(2)$ & 27 (1) \\
\hline$C(16)$ & $5301(3)$ & $-2496(2)$ & $3599(2)$ & $33(1)$ \\
\hline C (17) & $5635(3)$ & $-2509(2)$ & $4333(2)$ & $39(1)$ \\
\hline $\mathrm{C}(18)$ & $5410(3)$ & $-1736(2)$ & $4813(2)$ & $44(1)$ \\
\hline C (19) & $4839(3)$ & $-933(2)$ & $4550(2)$ & $34(1)$ \\
\hline$C(20)$ & $4249(2)$ & $1202(2)$ & $3855(2)$ & $22(1)$ \\
\hline$C(21)$ & $5156(2)$ & $1605(2)$ & $3495(2)$ & $28(1)$ \\
\hline C (22) & $5614(3)$ & $2453(2)$ & $3760(2)$ & $32(1)$ \\
\hline$C(23)$ & $5192(3)$ & $2917(2)$ & $4387(2)$ & $32(1)$ \\
\hline$C(24)$ & $4302(3)$ & $2520(2)$ & $4752(2)$ & $32(1)$ \\
\hline$C(25)$ & $3828(2)$ & $1666(2)$ & $4488(2)$ & $29(1)$ \\
\hline$C(26)$ & $885(2)$ & $1001(2)$ & $2501(2)$ & $24(1)$ \\
\hline$C(27)$ & $614(3)$ & $1204(2)$ & $1682(2)$ & $32(1)$ \\
\hline C (28) & $1405(2)$ & $1882(2)$ & $2888(2)$ & $25(1)$ \\
\hline$C(29)$ & $2340(2)$ & $2315(2)$ & $2610(2)$ & $29(1)$ \\
\hline$C(30)$ & $2788(3)$ & $3126(2)$ & $2961(2)$ & $35(1)$ \\
\hline C (31) & $2291(3)$ & $3534(2)$ & $3583(2)$ & $38(1)$ \\
\hline C (32) & $1355(3)$ & $3114(2)$ & $3875(2)$ & $40(1)$ \\
\hline C (33) & $920(3)$ & $2287(2)$ & $3533(2)$ & $32(1)$ \\
\hline
\end{tabular}

U(eq) is defined as $1 / 3$ the trace of the Uij tensor. 
Table S3-3. Bond lengths (A) and angles (deg) for 5e

$$
\begin{aligned}
& \mathrm{Pd}(1)-\mathrm{N}(9) \\
& \mathrm{Pd}(1)-\mathrm{C}(1) \\
& \mathrm{Pd}(1)-\mathrm{P}(1) \\
& \mathrm{P}(1)-\mathrm{C}(8) \\
& \mathrm{P}(1)-\mathrm{C}(1) \\
& \mathrm{P}(2)-\mathrm{C}(20) \\
& \mathrm{N}(9)-\mathrm{C}(26) \\
& \mathrm{C}(2)-\mathrm{C}(3) \\
& \mathrm{C}(4)-\mathrm{C}(5) \\
& \mathrm{C}(6)-\mathrm{C}(7) \\
& \mathrm{C}(8)-\mathrm{C}(13) \\
& \mathrm{C}(10)-\mathrm{C}(11) \\
& \mathrm{C}(12)-\mathrm{C}(13) \\
& \mathrm{C}(14)-\mathrm{C}(19) \\
& \mathrm{C}(16)-\mathrm{C}(17) \\
& \mathrm{C}(18)-\mathrm{C}(19) \\
& \mathrm{C}(20)-\mathrm{C}(21) \\
& \mathrm{C}(22)-\mathrm{C}(23) \\
& \mathrm{C}(24)-\mathrm{C}(25) \\
& \mathrm{C}(26)-\mathrm{C}(28) \\
& \mathrm{C}(28)-\mathrm{C}(33) \\
& \mathrm{C}(30)-\mathrm{C}(31) \\
& \mathrm{C}(32)-\mathrm{C}(33)
\end{aligned}
$$

$\mathrm{N}(9)-\mathrm{Pd}(1)-\mathrm{P}(2)$

$\mathrm{P}(2)-\mathrm{Pd}(1)-\mathrm{Cl}(1)$

$\mathrm{P}(2)-\mathrm{Pd}(1)-\mathrm{Cl}(2)$

$\mathrm{N}(9)-\mathrm{Pd}(1)-\mathrm{P}(1)$

$\mathrm{Cl}(1)-\mathrm{Pd}(1)-\mathrm{P}(1)$

$\mathrm{N}(9)-\mathrm{P}(1)-\mathrm{C}(8)$

$\mathrm{C}(8)-\mathrm{P}(1)-\mathrm{C}(2)$

$\mathrm{C}(8)-\mathrm{P}(1)-\mathrm{C}(1)$

$\mathrm{N}(9)-\mathrm{P}(1)-\mathrm{Pd}(1)$

$\mathrm{C}(2)-\mathrm{P}(1)-\mathrm{Pd}(1)$

$\mathrm{C}(14)-\mathrm{P}(2)-\mathrm{C}(20)$

$\mathrm{C}(20)-\mathrm{P}(2)-\mathrm{C}(1)$

$\mathrm{C}(20)-\mathrm{P}(2)-\mathrm{Pd}(1)$

$\mathrm{C}(26)-\mathrm{N}(9)-\mathrm{P}(1)$

$\mathrm{P}(1)-\mathrm{N}(9)-\mathrm{Pd}(1)$

$\mathrm{C}(7)-\mathrm{C}(2)-\mathrm{C}(3)$

$\mathrm{C}(3)-\mathrm{C}(2)-\mathrm{P}(1)$

$C(5)-C(4)-C(3)$

$C(5)-C(6)-C(7)$

$C(9)-C(8)-C(13)$

$\mathrm{C}(13)-\mathrm{C}(8)-\mathrm{P}(1)$

$C(11)-C(10)-C(9)$

$C(11)-C(12)-C(13)$

$C(15)-C(14)-C(19)$

$\mathrm{C}(19)-\mathrm{C}(14)-\mathrm{P}(2)$

$C(17)-C(16)-C(15)$

$\mathrm{C}(17)-\mathrm{C}(18)-\mathrm{C}(19)$

$C(25)-C(20)-C(21)$

$\mathrm{C}(21)-\mathrm{C}(20)-\mathrm{P}(2)$

$C(21)-C(22)-C(23)$

$C(23)-C(24)-C(25)$

$\mathrm{N}(9)-\mathrm{C}(26)-\mathrm{C}(27)$

$\mathrm{C}(27)-\mathrm{C}(26)-\mathrm{C}(28)$

$C(29)-C(28)-C(26)$

$C(30)-C(29)-C(28)$

$\mathrm{C}(30)-\mathrm{C}(31)-\mathrm{C}(32)$

C $(32)-C(33)-C(28)$
$2.094(2) \quad \mathrm{Pd}(1)-\mathrm{P}(2)$

2.3265(6 $\quad \mathrm{Pd}(1)-\mathrm{Cl}(2)$

$2.9742(7$

$1.799(3)$

$1.805(3)$

$1.820(3)$

$1.499(3)$

$1.398(4)$

$1.383(4)$

$1.380(4)$

$1.393(4)$

$1.379(4)$

$1.385(4)$

$1.401(4)$

$1.360(5)$

$1.383(4)$

$1.399(4)$

$1.377(4)$

$1.385(4)$

$1.525(4)$

$1.398(4)$

$1.375(5)$

$1.388(4)$

$89.35(6)$

$89.04(2)$

$175.78(3)$

$31.10(6)$

$147.62(2)$

$108.3(1)$

$109.5(1)$

$108.5(1)$

$42.49(8)$

$156.7(1)$

$106.2(1)$

$104.7(1)$

119.7 (1)

$119.2(2)$

$106.4(1)$

$119.5(3)$

$119.7(2)$

$120.0(3)$

$120.7(3)$

$120.2(2)$

$120.6(2)$

$120.3(3)$

$120.5(3)$

$119.4(3)$

$119.2(2)$

$120.2(3)$

$120.0(3)$

$119.0(2)$

$119.0(2)$

$120.5(3)$

$120.5(3)$

$110.8(2)$

$112.1(2)$

$121.8(3)$

$121.0(3)$

$120.1(3)$

$120.6(3)$

$\mathrm{P}(1)-\mathrm{N}(9)$

$P(1)-C(2)$

$\mathrm{P}(2)-\mathrm{C}(14)$

$P(2)-C(1)$

C (2) $-\mathrm{C}(7)$

$C(3)-C(4)$

C (5) $-\mathrm{C}(6)$

$C(8)-C(9)$

$C(9)-C(10)$

C (11) -C (12)

$C(14)-C(15)$

$\mathrm{C}(15)-\mathrm{C}(16)$

$C(17)-C(18)$

$C(20)-C(25)$

$C(21)-C(22)$

$C(23)-C(24)$

$C(26)-C(27)$

C $(28)-C(29)$

$C(29)-C(30)$

C (31) $-\mathrm{C}(32)$
$2.2425(6)$
$2.3687(7)$
$1.601(2)$
$1.805(3)$
$1.813(3)$
$1.834(2)$
$1.388(4)$
$1.389(4)$
$1.379(4)$
$1.391(4)$
$1.393(4)$
$1.384(5)$
$1.392(4)$
$1.381(4)$
$1.382(5)$
$1.385(4)$
$1.369(4)$
$1.383(4)$
$1.508(4)$
$1.386(4)$
$1.383(4)$
$1.388(5)$

$\mathrm{N}(9)-\mathrm{Pd}(1)-\mathrm{Cl}(1)$

$\mathrm{N}(9)-\mathrm{Pd}(1)-\mathrm{Cl}(2)$

$\mathrm{Cl}(1)-\mathrm{Pd}(1)-\mathrm{Cl}(2)$

$\mathrm{P}(2)-\mathrm{Pd}(1)-\mathrm{P}(1)$

$\mathrm{Cl}(2)-\mathrm{Pd}(1)-\mathrm{P}(1)$

$\mathrm{N}(9)-\mathrm{P}(1)-\mathrm{C}(2)$

$\mathrm{N}(9)-\mathrm{P}(1)-\mathrm{C}(1)$

$C(2)-P(1)-C(1)$

$\mathrm{C}(8)-\mathrm{P}(1)-\mathrm{Pd}(1)$

$\mathrm{C}(1)-\mathrm{P}(1)-\mathrm{Pd}(1)$

$\mathrm{C}(14)-\mathrm{P}(2)-\mathrm{C}(1)$

$\mathrm{C}(14)-\mathrm{P}(2)-\mathrm{Pd}(1)$

$\mathrm{C}(1)-\mathrm{P}(2)-\mathrm{Pd}(1)$

$\mathrm{C}(26)-\mathrm{N}(9)-\mathrm{Pd}(1)$

$\mathrm{P}(1)-\mathrm{C}(1)-\mathrm{P}(2)$

$C(7)-C(2)-P(1)$

$C(4)-C(3)-C(2)$

$C(6)-C(5)-C(4)$

$C(6)-C(7)-C(2)$

$C(9)-C(8)-P(1)$

$\mathrm{C}(8)-\mathrm{C}(9)-\mathrm{C}(10)$

$C(10)-C(11)-C(12)$

$C(12)-C(13)-C(8)$

$\mathrm{C}(15)-\mathrm{C}(14)-\mathrm{P}(2)$

$C(16)-C(15)-C(14)$

$\mathrm{C}(16)-\mathrm{C}(17)-\mathrm{C}(18)$

$C(18)-C(19)-C(14)$

$\mathrm{C}(25)-\mathrm{C}(20)-\mathrm{P}(2)$

C (22) $-\mathrm{C}(21)-\mathrm{C}(20)$

$C(22)-C(23)-C(24)$

$\mathrm{C}(24)-\mathrm{C}(25)-\mathrm{C}(20)$

$\mathrm{N}(9)-\mathrm{C}(26)-\mathrm{C}(28)$

$C(29)-C(28)-C(33)$

$C(33)-C(28)-C(26)$

$C(31)-C(30)-C(29)$

$C(33)-C(32)-C(31)$
$178.39(6)$

$91.37(6)$

$90.23(2)$

$65.02(2)$

$114.17(2)$

$114.6(1)$

$108.6(1)$

$107.2(1)$

$86.4(1)$

$82.37(8)$

$102.9(1)$

$115.3(1)$

$106.14(8)$

$121.0(2)$

$104.1(1)$

$120.8(2)$

$119.8(3)$

$120.0(3)$

$120.0(3)$

$119.2(2)$

$119.5(3)$

$120.0(3)$

$119.5(3)$

$121.2(2)$

$120.0(3)$

$120.9(3)$

$119.4(3)$

$121.9(2)$

$120.5(3)$

$119.6(3)$

$119.9(3)$

$113.6(2)$

$118.4(3)$

$119.8(3)$

120.1 (3)

$119.7(3)$ 
Table S3-4. Anisotropic displacement parameters ( $A^{\wedge} 2 \times 10^{\wedge} 3$ ) for 5e

\begin{tabular}{|c|c|c|c|c|c|c|}
\hline atom & $\mathrm{U} 11$ & $\mathrm{U} 22$ & U33 & U23 & U13 & $\mathrm{U} 12$ \\
\hline $\mathrm{Pd}(1)$ & $21(1)$ & $23(1)$ & $17(1)$ & $0(1)$ & $1(1)$ & $0(1)$ \\
\hline $\mathrm{Cl}(1)$ & 21 (1) & $25(1)$ & $24(1)$ & $-1(1)$ & $5(1)$ & $0(1)$ \\
\hline $\mathrm{Cl}(2)$ & 31 (1) & $45(1)$ & $17(1)$ & $-2(1)$ & $0(1)$ & $6(1)$ \\
\hline P (1) & $20(1)$ & $23(1)$ & $19(1)$ & $-1(1)$ & $0(1)$ & $-2(1)$ \\
\hline$P(2)$ & $20(1)$ & $23(1)$ & $19(1)$ & $0(1)$ & $1(1)$ & $-1(1)$ \\
\hline $\mathrm{N}(9)$ & $20(1)$ & $25(1)$ & $21(1)$ & $1(1)$ & $2(1)$ & $1(1)$ \\
\hline C (1) & $23(1)$ & $27(1)$ & $19(1)$ & $-1(1)$ & $2(1)$ & $-1(1)$ \\
\hline$C(2)$ & 21 (1) & $24(1)$ & $23(1)$ & $0(1)$ & $1(1)$ & $-3(1)$ \\
\hline$C(3)$ & $26(1)$ & $32(1)$ & $26(1)$ & $-2(1)$ & $2(1)$ & $-2(1)$ \\
\hline$C(4)$ & $30(2)$ & $44(2)$ & $27(1)$ & $-7(1)$ & $11(1)$ & $-4(1)$ \\
\hline$C(5)$ & $26(2)$ & $47(2)$ & $36(2)$ & $-5(1)$ & $7(1)$ & $-5(1)$ \\
\hline$C(6)$ & $26(2)$ & $47(2)$ & $33(2)$ & $-3(1)$ & $2(1)$ & $-8(1)$ \\
\hline$C(7)$ & $24(2)$ & $39(2)$ & $26(2)$ & $-2(1)$ & $1(1)$ & $-4(1)$ \\
\hline$C(8)$ & $19(1)$ & $23(1)$ & $24(1)$ & $0(1)$ & $0(1)$ & $-4(1)$ \\
\hline$C(9)$ & $25(2)$ & $29(1)$ & $26(2)$ & $-1(1)$ & $1(1)$ & $-2(1)$ \\
\hline$C(10)$ & $32(2)$ & $33(1)$ & $35(2)$ & $-9(1)$ & $5(2)$ & $-3(1)$ \\
\hline C (11) & $30(2)$ & $25(1)$ & $52(2)$ & $1(1)$ & $7(2)$ & $-1(1)$ \\
\hline$C(12)$ & $32(2)$ & $27(1)$ & $45(2)$ & $8(1)$ & $-4(2)$ & $1(1)$ \\
\hline$C(13)$ & $29(2)$ & $30(1)$ & $25(1)$ & $4(1)$ & $-1(1)$ & $-3(1)$ \\
\hline$C(14)$ & $22(1)$ & $26(1)$ & $22(1)$ & $0(1)$ & $1(1)$ & $0(1)$ \\
\hline C (15) & $25(2)$ & $27(1)$ & $28(2)$ & $-1(1)$ & $1(1)$ & $1(1)$ \\
\hline$C(16)$ & $33(2)$ & $25(1)$ & $42(2)$ & $-3(1)$ & $9(2)$ & $2(1)$ \\
\hline C (17) & $34(2)$ & $38(2)$ & $45(2)$ & $12(2)$ & $4(2)$ & $11(1)$ \\
\hline$C(18)$ & $45(2)$ & $53(2)$ & $32(2)$ & $9(2)$ & $-7(2)$ & $16(2)$ \\
\hline C (19) & $37(2)$ & $38(2)$ & $27(2)$ & $-1(1)$ & $-3(1)$ & $13(1)$ \\
\hline$C(20)$ & $23(2)$ & $23(1)$ & $21(1)$ & $-2(1)$ & $-4(1)$ & $-2(1)$ \\
\hline$C(21)$ & $28(2)$ & $32(1)$ & $24(2)$ & $-2(1)$ & $3(1)$ & $-2(1)$ \\
\hline C (22) & $34(2)$ & $29(1)$ & $32(2)$ & $-1(1)$ & $3(1)$ & $-8(1)$ \\
\hline C (23) & $38(2)$ & $27(1)$ & $31(2)$ & $0(1)$ & $-6(1)$ & $-4(1)$ \\
\hline C (24) & $34(2)$ & $34(1)$ & $29(2)$ & $-8(1)$ & $-2(1)$ & $-1(1)$ \\
\hline C (25) & $29(2)$ & $33(1)$ & $24(1)$ & $-2(1)$ & $0(1)$ & $-4(1)$ \\
\hline$C(26)$ & 21 (1) & $26(1)$ & $25(1)$ & $-1(1)$ & $-1(1)$ & $2(1)$ \\
\hline C (27) & $31(2)$ & $35(2)$ & $31(2)$ & $0(1)$ & $-6(1)$ & $6(1)$ \\
\hline C (28) & 22 (1) & $25(1)$ & $26(2)$ & $4(1)$ & $1(1)$ & $4(1)$ \\
\hline C (29) & $26(2)$ & $29(1)$ & $32(2)$ & $4(1)$ & $2(1)$ & $3(1)$ \\
\hline$C(30)$ & $28(2)$ & 31 (1) & $46(2)$ & $9(1)$ & $-5(2)$ & $-3(1)$ \\
\hline C (31) & $48(2)$ & $30(1)$ & $37(2)$ & $0(1)$ & $-13(2)$ & $-6(1)$ \\
\hline C (32) & $49(2)$ & $36(2)$ & $35(2)$ & $-9(1)$ & $4(2)$ & $-1(2)$ \\
\hline C (33) & $34(2)$ & $32(2)$ & $30(2)$ & $-2(1)$ & $5(1)$ & $-4(1)$ \\
\hline
\end{tabular}

The anisotropic displacement factor exponent takes the form $2 \mathrm{pi}^{\wedge} 2\left[\mathrm{~h} \wedge 2 \mathrm{a}{ }^{\wedge} 2 \mathrm{U}(11)+\ldots+2 \mathrm{hka} \mathrm{k}^{\star}{ }^{\star} \mathrm{U}(12)\right]$ 
Table S3-5. Hydrogen Coordinates (A $\times 10^{\wedge} 4$ ) and equivalent isotropic displacement parameters $\left(A^{\wedge} 2 \times 10^{\wedge} 3\right)$ for $\mathbf{5 e}$

\begin{tabular}{|c|c|c|c|c|}
\hline atom & $x$ & $\mathrm{Y}$ & $\mathrm{z}$ & $\mathrm{U}(\mathrm{eq})$ \\
\hline $\mathrm{H}(1 \mathrm{~A})$ & 2509 & -553 & 4436 & 27 \\
\hline $\mathrm{H}(1 \mathrm{~B})$ & 2140 & 548 & 4229 & 27 \\
\hline $\mathrm{H}(3)$ & 651 & -105 & 4821 & 34 \\
\hline $\mathrm{H}(4)$ & -1064 & -38 & 5385 & 41 \\
\hline $\mathrm{H}(5)$ & -2608 & -392 & 4671 & 44 \\
\hline $\mathrm{H}(6)$ & -2435 & -908.9999 & 3422 & 43 \\
\hline $\mathrm{H}(7)$ & -736 & -1005 & 2855 & 36 \\
\hline $\mathrm{H}(9)$ & 1395 & -1728 & 1997 & 32 \\
\hline $\mathrm{H}(10)$ & 1851 & -3353.9998 & 1710 & 40 \\
\hline $\mathrm{H}(11)$ & 2541 & -4379 & 2647 & 42 \\
\hline $\mathrm{H}(12)$ & 2761 & -3799 & 3880 & 42 \\
\hline $\mathrm{H}(13)$ & 2301 & -2185 & 4182 & 34 \\
\hline $\mathrm{H}(15)$ & 4509 & -1691 & 2805 & 32 \\
\hline $\mathrm{H}(16)$ & 5457 & -3035 & 3277 & 40 \\
\hline $\mathrm{H}(17)$ & 6027 & -3058 & 4519 & 47 \\
\hline $\mathrm{H}(18)$ & 5648 & -1755 & 5325 & 52 \\
\hline $\mathrm{H}(19)$ & 4682 & -400 & 4878 & 41 \\
\hline $\mathrm{H}(21)$ & 5457 & 1289 & 3064 & 34 \\
\hline $\mathrm{H}(22)$ & 6227 & 2722 & 3510 & 38 \\
\hline $\mathrm{H}(23)$ & 5510 & 3506 & 4568 & 38 \\
\hline $\mathrm{H}(24)$ & 4013 & 2836 & 5188 & 39 \\
\hline $\mathrm{H}(25)$ & 3216 & 1399 & 4741 & 34 \\
\hline H (26) & 181 & 879 & 2766 & 29 \\
\hline $\mathrm{H}(27 \mathrm{~A})$ & 1284 & 1324 & 1399 & 48 \\
\hline $\mathrm{H}(27 \mathrm{~B})$ & 145 & 1782 & 1650 & 48 \\
\hline $\mathrm{H}(27 \mathrm{C})$ & 238 & 637 & 1465 & 48 \\
\hline $\mathrm{H}(29)$ & 2679 & 2050 & 2172 & 35 \\
\hline $\mathrm{H}(30)$ & 3441 & 3402 & 2771 & 42 \\
\hline $\mathrm{H}(31)$ & 2588 & 4103 & 3813 & 46 \\
\hline $\mathrm{H}(32)$ & 1014 & 3391 & 4308 & 48 \\
\hline $\mathrm{H}(33)$ & 286 & 1993 & 3738 & 39 \\
\hline
\end{tabular}


Figure S4: Molecular structure of 5'e

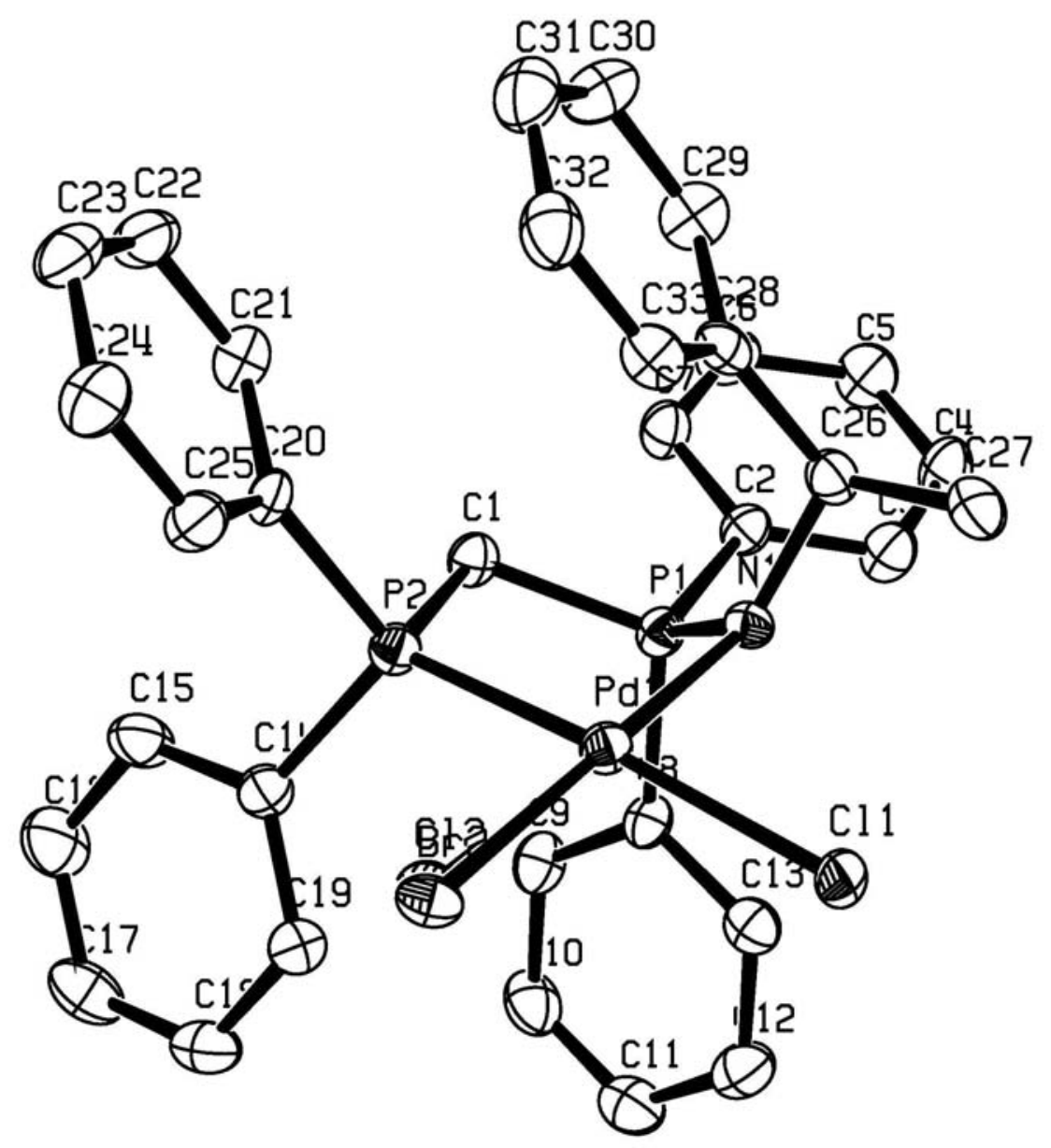


Table S4-1. Crystal data for 5'e

Compound

Molecular formula

Molecular weight

Crystal habit

Crystal dimensions(mm)

Crystal system

Space group

$\mathrm{a}(\AA)$

$\mathrm{b}(\AA)$

$\mathrm{c}(\AA)$

$\alpha\left(^{\circ}\right)$

$\beta\left(^{\circ}\right)$

$\gamma\left({ }^{\circ}\right)$

$\mathrm{V}\left(\AA^{3}\right)$

Z

$\mathrm{d}\left(\mathrm{g}-\mathrm{cm}^{-3}\right)$

$\mathrm{F}(000)$

$\mu\left(\mathrm{cm}^{-1}\right)$

Absorption corrections

Diffractometer

$\mathrm{X}$-ray source

$\lambda(\AA)$

Monochromator

$\mathrm{T}(\mathrm{K})$

Scan mode

Maximum $\theta$

HKL ranges

Reflections measured

Unique data

Rint

Reflections used

Criterion

Refinement type

Hydrogen atoms

Parameters refined

Reflections / parameter

wR2

R1

Flack's parameter

Weights $a, b$

GoF

difference peak / hole (e $\left.\AA^{-3}\right)$ 5'e

$\mathrm{C}_{33} \mathrm{H}_{31} \mathrm{Br}_{0.16} \mathrm{Cl}_{1.84} \mathrm{NP}_{2} \mathrm{Pd}$

688.05

orange plate

$0.12 \times 0.10 \times 0.04$

orthorhombic

$\mathrm{P} 2{ }_{1} 22_{1}$

$12.3220(10)$

$13.6670(10)$

$17.6790(10)$

90.00

90.00

90.00

2977.2(4)

4

1.535

1396

1.138

multi-scan ; $0.8755 \min , 0.9559 \max$

KappaCCD

$\mathrm{MoK} \alpha$

0.71069

graphite

150.0(1)

phi and omega scans

30.03

$-1717 ;-1919 ;-2424$

8674

8674

0.0000

6885

I $>2 \sigma \mathrm{I})$

Fsqd

mixed

364

18

0.0930

0.0398

$0.01(2)$

$0.0457 ; 0.0000$

1.005

$0.999(0.103) /-1.117(0.103)$ 
Table 4-2. Atomic Coordinates (A x 10^4) and equivalent isotropic displacement parameters $\left(A^{\wedge} 2 \times 10^{\wedge} 3\right)$ for 5'e

\begin{tabular}{|c|c|c|c|c|}
\hline atom & $\mathrm{x}$ & $\mathrm{Y}$ & $z$ & $\mathrm{U}(\mathrm{eq})$ \\
\hline $\mathrm{Pd}(1)$ & $1840(1)$ & $34(1)$ & $2752(1)$ & $19(1)$ \\
\hline $\mathrm{Cl}(1)$ & $2334(1)$ & $-161(1)$ & 4047 (1) & $24(1)$ \\
\hline $\mathrm{Br}(2)$ & $-40(20)$ & $0(10)$ & $3130(10)$ & $22(2)$ \\
\hline $\mathrm{Cl}(2)$ & $10(8)$ & $-20(5)$ & $3064(6)$ & $25(1)$ \\
\hline$P(1)$ & $3564(1)$ & $-555(1)$ & $1667(1)$ & $20(1)$ \\
\hline$P(2)$ & $1356(1)$ & $92(1)$ & $1529(1)$ & $20(1)$ \\
\hline $\mathrm{N}(1)$ & $3476(2)$ & $79(2)$ & $2431(1)$ & $19(1)$ \\
\hline$C(1)$ & $2610(2)$ & $-81(3)$ & $988(2)$ & $21(1)$ \\
\hline$C(2)$ & $4891(3)$ & $-540(3)$ & $1227(2)$ & $22(1)$ \\
\hline$C(3)$ & $5808(3)$ & $-794(3)$ & $1638(2)$ & $29(1)$ \\
\hline$C(4)$ & $6819(3)$ & $-728(3)$ & $1298(2)$ & $34(1)$ \\
\hline$C(5)$ & $6919(3)$ & $-437(3)$ & $562(2)$ & 35 (1) \\
\hline$C(6)$ & $6004(3)$ & $-223(3)$ & $139(2)$ & $32(1)$ \\
\hline$C(7)$ & $4993(3)$ & $-260(3)$ & $469(2)$ & $27(1)$ \\
\hline$C(8)$ & $3201(3)$ & -1795 (2) & $1881(2)$ & $21(1)$ \\
\hline$C(9)$ & $2801(3)$ & $-2415(3)$ & $1320(2)$ & $27(1)$ \\
\hline$C(10)$ & $2520(3)$ & $-3367(3)$ & $1497(2)$ & 34 (1) \\
\hline$C(11)$ & $2652(3)$ & $-3715(3)$ & $2222(2)$ & $33(1)$ \\
\hline$C(12)$ & $3062(3)$ & $-3111(3)$ & $2780(2)$ & $32(1)$ \\
\hline$C(13)$ & $3329(3)$ & $-2146(3)$ & $2613(2)$ & $25(1)$ \\
\hline$C(14)$ & $510(3)$ & $-910(3)$ & $1211(2)$ & $23(1)$ \\
\hline$C(15)$ & $169(3)$ & $-933(3)$ & $454(2)$ & $32(1)$ \\
\hline$C(16)$ & $-400(4)$ & $-1729(3)$ & $189(2)$ & $42(1)$ \\
\hline$C(17)$ & $-638(4)$ & $-2497(3)$ & $660(2)$ & $39(1)$ \\
\hline$C(18)$ & $-304(3)$ & $-2493(3)$ & $1402(2)$ & $31(1)$ \\
\hline$C(19)$ & $272(3)$ & $-1696(3)$ & $1675(2)$ & $26(1)$ \\
\hline$C(20)$ & $755(3)$ & $1202(3)$ & $1148(2)$ & $22(1)$ \\
\hline$C(21)$ & $1172(3)$ & $1661(3)$ & $516(2)$ & $27(1)$ \\
\hline$C(22)$ & $709(3)$ & 2517 (3) & $247(2)$ & $32(1)$ \\
\hline$C(23)$ & $-196(3)$ & $2909(3)$ & $609(2)$ & 31 (1) \\
\hline$C(24)$ & $-615(3)$ & $2446(3)$ & $1236(2)$ & $32(1)$ \\
\hline$C(25)$ & $-156(3)$ & $1603(3)$ & $1504(2)$ & $27(1)$ \\
\hline$C(26)$ & $4122(3)$ & $999(3)$ & $2499(2)$ & $23(1)$ \\
\hline$C(27)$ & $4392(3)$ & $1207(3)$ & $3315(2)$ & $30(1)$ \\
\hline$C(28)$ & $3600(3)$ & $1885(2)$ & $2111(2)$ & $22(1)$ \\
\hline$C(29)$ & $4087(3)$ & 2289 (3) & 1469 (2) & $32(1)$ \\
\hline$C(30)$ & $3649(4)$ & $3117(3)$ & $1129(2)$ & $39(1)$ \\
\hline$C(31)$ & $2712(4)$ & $3532(3)$ & $1417(3)$ & $38(1)$ \\
\hline$C(32)$ & $2218(3)$ & $3121(3)$ & $2043(2)$ & $34(1)$ \\
\hline$C(33)$ & $2664(3)$ & $2315(3)$ & $2391(2)$ & $28(1)$ \\
\hline
\end{tabular}

U(eq) is defined as $1 / 3$ the trace of the Uij tensor. 
Table S4-3. Bond lengths (A) and angles (deg) for 5'e

$$
\begin{aligned}
& \mathrm{Pd}(1)-\mathrm{N}(1) \\
& \mathrm{Pd}(1)-\mathrm{C}(2) \\
& \mathrm{Pd}(1)-\mathrm{Br}(2) \\
& \mathrm{P}(1)-\mathrm{N}(1) \\
& \mathrm{P}(1)-\mathrm{C}(1) \\
& \mathrm{P}(2)-\mathrm{C}(14) \\
& \mathrm{P}(2)-\mathrm{C}(1) \\
& \mathrm{C}(2)-\mathrm{C}(3) \\
& \mathrm{C}(3)-\mathrm{C}(4) \\
& \mathrm{C}(5)-\mathrm{C}(6) \\
& \mathrm{C}(8)-\mathrm{C}(13) \\
& \mathrm{C}(9)-\mathrm{C}(10) \\
& \mathrm{C}(11)-\mathrm{C}(12) \\
& \mathrm{C}(14)-\mathrm{C}(19) \\
& \mathrm{C}(15)-\mathrm{C}(16) \\
& \mathrm{C}(17)-\mathrm{C}(18) \\
& \mathrm{C}(20)-\mathrm{C}(21) \\
& \mathrm{C}(21)-\mathrm{C}(22) \\
& \mathrm{C}(23)-\mathrm{C}(24) \\
& \mathrm{C}(26)-\mathrm{C}(27) \\
& \mathrm{C}(28)-\mathrm{C}(33) \\
& \mathrm{C}(29)-\mathrm{C}(30) \\
& \mathrm{C}(31)-\mathrm{C}(32)
\end{aligned}
$$

$\mathrm{N}(1)-\mathrm{Pd}(1)-\mathrm{P}(2)$

$\mathrm{P}(2)-\mathrm{Pd}(1)-\mathrm{Cl}(2)$

$P(2)-P d(1)-C l(1)$

$\mathrm{N}(1)-\mathrm{Pd}(1)-\mathrm{Br}(2)$

$\mathrm{Cl}(2)-\mathrm{Pd}(1)-\mathrm{Br}(2)$

$\mathrm{N}(1)-\mathrm{Pd}(1)-\mathrm{P}(1)$

$\mathrm{Cl}(2)-\mathrm{Pd}(1)-\mathrm{P}(1)$

$\operatorname{Br}(2)-P d(1)-P(1)$

$\mathrm{N}(1)-\mathrm{P}(1)-\mathrm{C}(1)$

$\mathrm{N}(1)-\mathrm{P}(1)-\mathrm{C}(2)$

$C(1)-P(1)-C(2)$

$\mathrm{C}(8)-\mathrm{P}(1)-\mathrm{Pd}(1)$

$\mathrm{C}(2)-\mathrm{P}(1)-\mathrm{Pd}(1)$

$\mathrm{C}(14)-\mathrm{P}(2)-\mathrm{C}(1)$

$\mathrm{C}(14)-\mathrm{P}(2)-\mathrm{Pd}(1)$

$\mathrm{C}(1)-\mathrm{P}(2)-\mathrm{Pd}(1)$

$\mathrm{C}(26)-\mathrm{N}(1)-\mathrm{Pd}(1)$

$\mathrm{P}(1)-\mathrm{C}(1)-\mathrm{P}(2)$

$\mathrm{C}(3)-\mathrm{C}(2)-\mathrm{P}(1)$

$C(4)-C(3)-C(2)$

$C(4)-C(5)-C(6)$

$C(6)-C(7)-C(2)$

$\mathrm{C}(13)-\mathrm{C}(8)-\mathrm{P}(1)$

$C(10)-C(9)-C(8)$

$C(10)-C(11)-C(12)$

$\mathrm{C}(8)-\mathrm{C}(13)-\mathrm{C}(12)$

$\mathrm{C}(19)-\mathrm{C}(14)-\mathrm{P}(2)$

$\mathrm{C}(16)-\mathrm{C}(15)-\mathrm{C}(14)$

$C(16)-C(17)-C(18)$

$\mathrm{C}(14)-\mathrm{C}(19)-\mathrm{C}(18)$

$\mathrm{C}(21)-\mathrm{C}(20)-\mathrm{P}(2)$

$\mathrm{C}(20)-\mathrm{C}(21)-\mathrm{C}(22)$

C (24) $-\mathrm{C}(23)-\mathrm{C}(22)$

$C(24)-C(25)-C(20)$

$\mathrm{N}(1)-\mathrm{C}(26)-\mathrm{C}(28)$

C (33) $-\mathrm{C}(28)-\mathrm{C}(29)$

C (29) $-C(28)-C(26)$

$C(31)-C(30)-C(29)$

C (33) -C (32) -C (31)

$\begin{array}{ll}2.095(2) & \mathrm{Pd}(1)-\mathrm{P}(2) \\ 2.323(9) & \mathrm{Pd}(1)-\mathrm{C}(1) \\ 2.41(2) & \mathrm{Pd}(1)-\mathrm{P}(1) \\ 1.608(3) & \mathrm{P}(1)-\mathrm{C}(8) \\ 1.801(3) & \mathrm{P}(1)-\mathrm{C}(2) \\ 1.810(4) & \mathrm{P}(2)-\mathrm{C}(20) \\ 1.833(3) & \mathrm{N}(1)-\mathrm{C}(26) \\ 1.387(5) & \mathrm{C}(2)-\mathrm{C}(7) \\ 1.386(5) & \mathrm{C}(4)-\mathrm{C}(5) \\ 1.384(5) & \mathrm{C}(6)-\mathrm{C}(7) \\ 1.390(5) & \mathrm{C}(8)-\mathrm{C}(9) \\ 1.382(6) & \mathrm{C}(10)-\mathrm{C}(11) \\ 1.383(5) & \mathrm{C}(12)-\mathrm{C}(13) \\ 1.383(5) & \mathrm{C}(14)-\mathrm{C}(15) \\ 1.375(5) & \mathrm{C}(16)-\mathrm{C}(17) \\ 1.374(6) & \mathrm{C}(18)-\mathrm{C}(19) \\ 1.382(5) & \mathrm{C}(20)-\mathrm{C}(25) \\ 1.385(5) & \mathrm{C}(22)-\mathrm{C}(23) \\ 1.377(5) & \mathrm{C}(24)-\mathrm{C}(25) \\ 1.507(5) & \mathrm{C}(26)-\mathrm{C}(28) \\ 1.386(5) & \mathrm{C}(28)-\mathrm{C}(29) \\ 1.390(5) & \mathrm{C}(30)-\mathrm{C}(31) \\ 1.381(6) & \mathrm{C}(32)-\mathrm{C}(33)\end{array}$

$2.2444(8)$

$2.3830(8)$

$2.974(1)$

$1.793(3)$

$1.810(3)$

$1.819(4)$

$1.494(4)$

$1.400(5)$

$1.367(5)$

$1.376(5)$

1.394(5)

$1.376(6)$

$1.391(5)$

$1.403(5)$

$1.372(6)$

$1.387(5)$

$1.398(5)$

$1.393(5)$

$1.368(5)$

$1.534(5)$

$1.397(5)$

$1.384(6)$

$1.377(5)$
$89.61(7)$

$88.4(3)$

$175.55(4)$

$179.3(4)$

$2.6(7)$

$31.31(7)$

$146.9(2)$

$149.4(4)$

$108.8(2)$

$114.6(2)$

$107.4(2)$

$86.7(1)$

$156.8(1)$

$103.0(2)$

$115.2(1)$

$106.0(1)$

$121.1(2)$

$104.4(2)$

$120.5(2)$

$119.2(3)$

$120.1(4)$

$119.8(3)$

$119.6(3)$

$119.9(3)$

$120.1(4)$

$119.9(3)$

$121.7(3)$

$119.7(4)$

$120.8(4)$

$120.8(4)$

$121.8(3)$

$120.5(3)$

$119.6(4)$

$120.4(4)$

$113.9(3)$

$118.7(3)$

$119.7(3)$

$119.9(4)$

$120.5(4)$
$\mathrm{N}(1)-\mathrm{Pd}(1)-\mathrm{Cl}(2)$

$\mathrm{N}(1)-\mathrm{Pd}(1)-\mathrm{Cl}(1)$

$\mathrm{Cl}(2)-\mathrm{Pd}(1)-\mathrm{Cl}(1)$

$P(2)-P d(1)-B r(2)$

$\mathrm{Cl}(1)-\mathrm{Pd}(1)-\mathrm{Br}(2)$

$\mathrm{P}(2)-\mathrm{Pd}(1)-\mathrm{P}(1)$

$\mathrm{Cl}(1)-\mathrm{Pd}(1)-\mathrm{P}(1)$

$\mathrm{N}(1)-\mathrm{P}(1)-\mathrm{C}(8)$

$\mathrm{C}(8)-\mathrm{P}(1)-\mathrm{C}(1)$

$\mathrm{C}(8)-\mathrm{P}(1)-\mathrm{C}(2)$

$\mathrm{N}(1)-\mathrm{P}(1)-\mathrm{Pd}(1)$

$\mathrm{C}(1)-\mathrm{P}(1)-\mathrm{Pd}(1)$

$C(14)-P(2)-C(20)$

$\mathrm{C}(20)-\mathrm{P}(2)-\mathrm{C}(1)$

$\mathrm{C}(20)-\mathrm{P}(2)-\mathrm{Pd}(1)$

$\mathrm{C}(26)-\mathrm{N}(1)-\mathrm{P}(1)$

$\mathrm{P}(1)-\mathrm{N}(1)-\mathrm{Pd}(1)$

$\mathrm{C}(3)-\mathrm{C}(2)-\mathrm{C}(7)$

$C(7)-C(2)-P(1)$

$C(5)-C(4)-C(3)$

$\mathrm{C}(7)-\mathrm{C}(6)-\mathrm{C}(5)$

$C(13)-C(8)-C(9)$

$\mathrm{C}(9)-\mathrm{C}(8)-\mathrm{P}(1)$

$C(11)-C(10)-C(9)$

$C(11)-C(12)-C(13)$

$C(19)-C(14)-C(15)$

$\mathrm{C}(15)-\mathrm{C}(14)-\mathrm{P}(2)$

$C(17)-C(16)-C(15)$

$C(17)-C(18)-C(19)$

$C(21)-C(20)-C(25)$

$C(25)-C(20)-P(2)$

$C(21)-C(22)-C(23)$

$C(25)-C(24)-C(23)$

$\mathrm{N}(1)-\mathrm{C}(26)-\mathrm{C}(27)$

$\mathrm{C}(27)-\mathrm{C}(26)-\mathrm{C}(28)$

$C(33)-C(28)-C(26)$

$C(30)-C(29)-C(28)$

C (32) $-\mathrm{C}(31)-\mathrm{C}(30)$

C (32) $-\mathrm{C}(33)-\mathrm{C}(28)$
$178.0(3)$

$91.02(7)$

$91.0(3)$

$90.9(5)$

$88.5(5)$

$65.01(3)$

$114.00(3)$

$108.4(2)$

$108.5(2)$

$109.1(2)$

$42.6(1)$

$82.3(1)$

$106.3(2)$

104.9 (2)

$119.6(1)$

$119.0(2)$

$106.1(1)$

$119.8(3)$

$119.7(3)$

$120.9(4)$

$120.1(3)$

$119.6(3)$

$120.8(3)$

$120.5(4)$

$120.0(3)$

$119.0(4)$

119.1 (3)

$120.5(4)$

$119.2(4)$

$119.0(3)$

$119.2(3)$

$119.8(3)$

$120.7(4)$

110.7 (3)

$111.8(3)$

$121.6(3)$

$120.4(4)$

$119.8(4)$

$120.8(4)$ 
Table S4-4. Anisotropic displacement parameters (A^2 x 10^3) for 5'e

\begin{tabular}{|c|c|c|c|c|c|c|}
\hline atom & U11 & $\mathrm{U} 22$ & U33 & $\mathrm{U} 23$ & U13 & $\mathrm{U} 12$ \\
\hline $\mathrm{Pd}(1)$ & $20(1)$ & $22(1)$ & 17 (1) & $0(1)$ & $1(1)$ & $0(1)$ \\
\hline $\mathrm{Cl}(1)$ & $24(1)$ & $36(1)$ & 11 (1) & $1(1)$ & $0(1)$ & $-6(1)$ \\
\hline $\operatorname{Br}(2)$ & $23(3)$ & $19(3)$ & $22(3)$ & $-2(2)$ & $7(2)$ & $-2(3)$ \\
\hline $\mathrm{Cl}(2)$ & $22(1)$ & $30(2)$ & $25(2)$ & $0(1)$ & $5(1)$ & $3(1)$ \\
\hline P (1) & $19(1)$ & $22(1)$ & $18(1)$ & $1(1)$ & $1(1)$ & $2(1)$ \\
\hline P (2) & $19(1)$ & 22 (1) & $19(1)$ & $1(1)$ & $1(1)$ & $1(1)$ \\
\hline $\mathrm{N}(1)$ & $17(1)$ & 21 (1) & $18(1)$ & $2(1)$ & $1(1)$ & $-3(1)$ \\
\hline$C(1)$ & $23(1)$ & $25(2)$ & $16(1)$ & $-1(2)$ & $1(1)$ & $1(2)$ \\
\hline C (2) & $22(2)$ & $22(2)$ & $23(2)$ & $1(1)$ & $0(1)$ & $3(1)$ \\
\hline C (3) & $25(2)$ & $36(2)$ & $26(2)$ & $4(2)$ & $2(2)$ & $5(2)$ \\
\hline C (4) & $24(2)$ & $44(2)$ & $35(2)$ & $3(2)$ & $-3(2)$ & $10(2)$ \\
\hline C ( 5$)$ & $22(2)$ & $47(2)$ & $37(2)$ & $4(2)$ & $8(2)$ & $5(2)$ \\
\hline$C(6)$ & $30(2)$ & $39(3)$ & $27(2)$ & $7(2)$ & $11(2)$ & $3(2)$ \\
\hline$C(7)$ & $23(2)$ & $33(2)$ & $26(2)$ & $2(1)$ & $-2(1)$ & $3(1)$ \\
\hline C ( 8) & $22(2)$ & $19(2)$ & $22(2)$ & $-1(1)$ & $-2(2)$ & $6(2)$ \\
\hline C (9) & $30(2)$ & $29(2)$ & $23(2)$ & $-4(2)$ & $-3(2)$ & $5(2)$ \\
\hline$C(10)$ & $33(2)$ & 25 (2) & $44(3)$ & $-7(2)$ & $-8(2)$ & $-2(2)$ \\
\hline$C(11)$ & $27(2)$ & $23(2)$ & $48(2)$ & $1(2)$ & $4(2)$ & $2(2)$ \\
\hline$C(12)$ & $31(2)$ & $33(2)$ & $32(2)$ & $9(2)$ & $3(2)$ & $5(2)$ \\
\hline$C(13)$ & $22(2)$ & $25(2)$ & $28(2)$ & $-2(1)$ & $2(2)$ & $1(1)$ \\
\hline$C(14)$ & $24(2)$ & $26(2)$ & $19(2)$ & $0(1)$ & $2(2)$ & $-1(2)$ \\
\hline$C(15)$ & $36(2)$ & $36(2)$ & $25(2)$ & $3(2)$ & $2(2)$ & $-12(2)$ \\
\hline$C(16)$ & $46(3)$ & $50(3)$ & $29(2)$ & $-5(2)$ & $-5(2)$ & $-14(2)$ \\
\hline C (17) & $38(2)$ & $32(2)$ & $45(2)$ & $-12(2)$ & $5(2)$ & $-12(2)$ \\
\hline$C(18)$ & $30(2)$ & $23(2)$ & $41(2)$ & $4(2)$ & $9(2)$ & $0(2)$ \\
\hline C (19) & $27(2)$ & $26(2)$ & $26(2)$ & $2(2)$ & $0(2)$ & $-1(2)$ \\
\hline$C(20)$ & $20(2)$ & $22(2)$ & $23(2)$ & $4(1)$ & $-6(1)$ & $4(1)$ \\
\hline$C(21)$ & $27(2)$ & $29(2)$ & $23(2)$ & $-1(1)$ & $-1(2)$ & $6(2)$ \\
\hline$C(22)$ & $36(2)$ & $29(2)$ & $29(2)$ & $9(2)$ & $1(2)$ & $1(2)$ \\
\hline C (23) & $43(2)$ & $22(2)$ & $29(2)$ & $2(2)$ & $-5(2)$ & $5(2)$ \\
\hline C (24) & $34(2)$ & $28(2)$ & $34(2)$ & $-3(2)$ & $1(2)$ & $10(2)$ \\
\hline C (25) & $27(2)$ & $28(2)$ & $27(2)$ & $5(2)$ & $1(2)$ & $3(2)$ \\
\hline C (26) & $20(2)$ & 25 (2) & $22(2)$ & $-1(1)$ & $0(1)$ & $-3(1)$ \\
\hline C (27) & $31(2)$ & $30(2)$ & $29(2)$ & $-2(2)$ & $-5(2)$ & $-8(2)$ \\
\hline$C(28)$ & $23(2)$ & $22(2)$ & $23(2)$ & $-4(1)$ & $0(2)$ & $-6(2)$ \\
\hline C (29) & $34(2)$ & $32(2)$ & $30(2)$ & $-2(2)$ & $4(2)$ & $5(2)$ \\
\hline$C(30)$ & $49(3)$ & $35(2)$ & $32(2)$ & $11(2)$ & $0(2)$ & $3(2)$ \\
\hline$C(31)$ & $45(3)$ & $27(2)$ & $43(2)$ & $-4(2)$ & $-14(2)$ & $7(2)$ \\
\hline$C(32)$ & $31(2)$ & $30(2)$ & $41(2)$ & $-6(2)$ & $-9(2)$ & $5(2)$ \\
\hline C (33) & $27(2)$ & $28(2)$ & $30(2)$ & $-3(2)$ & $1(2)$ & $-2(2)$ \\
\hline
\end{tabular}

The anisotropic displacement factor exponent takes the form $2 \mathrm{pi}^{\wedge} 2\left[\mathrm{~h}^{\wedge} 2 \mathrm{a} \star \wedge 2 \mathrm{U}(11)+\ldots+2 \mathrm{hka} * \mathrm{~b} * \mathrm{U}(12)\right]$ 
Table S4-5. Hydrogen Coordinates (A $\times 10^{\wedge} 4$ ) and equivalent isotropic displacement parameters $\left(A^{\wedge} 2 \times 10^{\wedge} 3\right)$ for $\mathbf{5}^{\prime} \mathbf{e}$

\begin{tabular}{|c|c|c|c|c|}
\hline atom & $\mathrm{x}$ & $\mathrm{Y}$ & $\mathrm{z}$ & $\mathrm{U}(\mathrm{eq})$ \\
\hline $\mathrm{H}(1 \mathrm{~A})$ & 2497 & -552 & 569 & 26 \\
\hline $\mathrm{H}(1 \mathrm{~B})$ & 2867 & 547 & 775 & 26 \\
\hline $\mathrm{H}(3)$ & 5743 & -1011 & 2147 & 35 \\
\hline $\mathrm{H}(4)$ & 7450 & -886.9999 & 1581 & 41 \\
\hline $\mathrm{H}(5)$ & 7619 & -382.0000 & 339 & 42 \\
\hline $\mathrm{H}(6)$ & 6074 & -50 & -379 & 39 \\
\hline $\mathrm{H}(7)$ & 4366 & -97 & 182 & 33 \\
\hline $\mathrm{H}(9)$ & 2721 & -2183 & 816 & 33 \\
\hline $\mathrm{H}(10)$ & 2235 & -3784 & 1116 & 41 \\
\hline $\mathrm{H}(11)$ & 2460 & -4371 & 2339 & 39 \\
\hline $\mathrm{H}(12)$ & 3162 & -3355 & 3278 & 38 \\
\hline $\mathrm{H}(13)$ & 3599 & -1728 & 2998 & 30 \\
\hline $\mathrm{H}(15)$ & 329 & -402 & 126 & 38 \\
\hline $\mathrm{H}(16)$ & -629 & -1746 & -324 & 50 \\
\hline $\mathrm{H}(17)$ & -1038 & -3037.9998 & 471 & 46 \\
\hline $\mathrm{H}(18)$ & -467 & -3028.9998 & 1724 & 38 \\
\hline $\mathrm{H}(19)$ & 507 & -1690 & 2187 & 32 \\
\hline $\mathrm{H}(21)$ & 1782 & 1388 & 263 & 32 \\
\hline $\mathrm{H}(22)$ & 1007 & 2834 & -183 & 38 \\
\hline $\mathrm{H}(23)$ & -522 & 3492 & 424 & 38 \\
\hline $\mathrm{H}(24)$ & -1229 & 2716 & 1485 & 38 \\
\hline $\mathrm{H}(25)$ & -458 & 1289 & 1934 & 33 \\
\hline $\mathrm{H}(26)$ & 4827 & 878 & 2235 & 27 \\
\hline $\mathrm{H}(27 \mathrm{~A})$ & 3721 & 1322 & 3599 & 45 \\
\hline $\mathrm{H}(27 \mathrm{~B})$ & 4855 & 1789 & 3345 & 45 \\
\hline $\mathrm{H}(27 \mathrm{C})$ & 4777 & 646 & 3532 & 45 \\
\hline $\mathrm{H}(29)$ & 4720 & 1995 & 1263 & 38 \\
\hline $\mathrm{H}(30)$ & 3993 & 3398 & 700 & 46 \\
\hline $\mathrm{H}(31)$ & 2410 & 4097 & 1186 & 46 \\
\hline $\mathrm{H}(32)$ & 1565 & 3397 & 2234 & 41 \\
\hline $\mathrm{H}(33)$ & 2326 & 2050 & 2829 & 34 \\
\hline
\end{tabular}


Figure S5: Molecular structure of $\mathbf{6 c}$

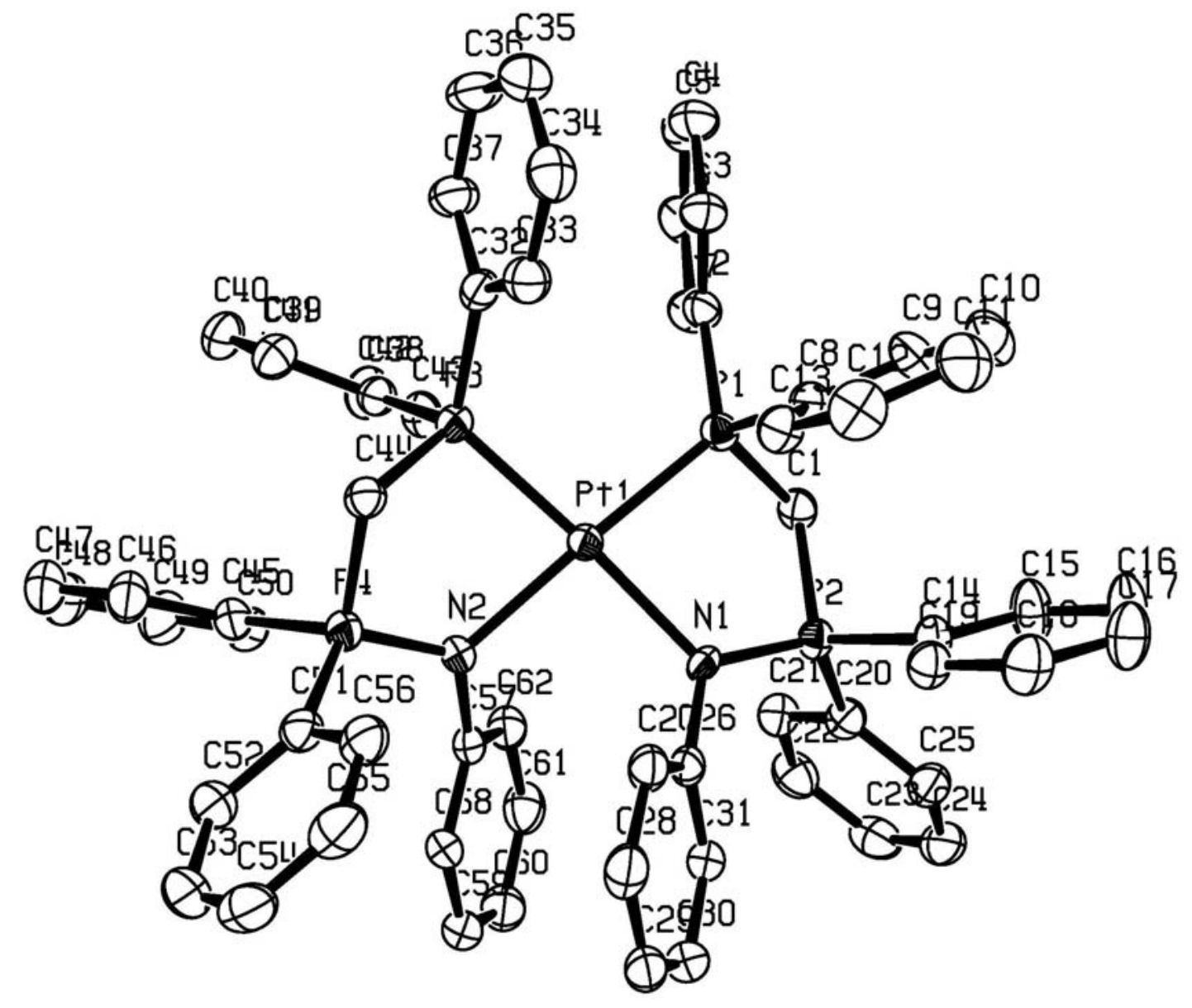


Table S5-1. Crystal data for $\mathbf{6 c}$

\begin{tabular}{|c|c|}
\hline Compound & 6c \\
\hline Molecular formula & $\mathrm{C}_{62} \mathrm{H}_{54} \mathrm{~N}_{2} \mathrm{P}_{4} \mathrm{Pt}, 2.5\left(\mathrm{CH}_{2} \mathrm{Cl}_{2}\right),(\mathrm{Br}),(\mathrm{Cl})$ \\
\hline Molecular weight & 1473.72 \\
\hline Crystal habit & colorless block \\
\hline Crystal dimensions $(\mathrm{mm})$ & $0.20 \times 0.20 \times 0.20$ \\
\hline Crystal system & triclinic \\
\hline Space group & Pbar1 \\
\hline $\mathrm{a}(\AA)$ & $12.4100(10)$ \\
\hline $\mathrm{b}(\AA)$ & $12.6440(10)$ \\
\hline$c(\AA)$ & $21.6810(10)$ \\
\hline$\alpha\left(^{\circ}\right)$ & $75.6000(10)$ \\
\hline$\beta\left(^{\circ}\right)$ & $75.8500(10)$ \\
\hline$\gamma\left({ }^{\circ}\right)$ & $81.0400(10)$ \\
\hline $\mathrm{V}\left(\AA^{3}\right)$ & $3178.8(4)$ \\
\hline $\mathrm{Z}$ & 2 \\
\hline $\mathrm{d}\left(\mathrm{g}-\mathrm{cm}^{-3}\right)$ & 1.540 \\
\hline F000 & 1470 \\
\hline$\mu\left(\mathrm{cm}^{-1}\right)$ & 3.229 \\
\hline Absorption corrections & multi-scan ; $0.5644 \min , 0.5644 \max$ \\
\hline Diffractometer & KappaCCD \\
\hline X-ray source & $\operatorname{MoK} \alpha$ \\
\hline$\lambda(\AA)$ & 0.71069 \\
\hline Monochromator & graphite \\
\hline $\mathrm{T}(\mathrm{K})$ & $150.0(1)$ \\
\hline Scan mode & phi and omega scans \\
\hline Maximum $\theta$ & 30.03 \\
\hline HKL ranges & $-1712 ;-1717 ;-3029$ \\
\hline Reflections measured & 25128 \\
\hline Unique data & 18447 \\
\hline Rint & 0.0209 \\
\hline Reflections used & 15385 \\
\hline Criterion & $>2 \operatorname{sigma}(\mathrm{I})$ \\
\hline Refinement type & Fsqd \\
\hline Hydrogen atoms & mixed \\
\hline Parameters refined & 722 \\
\hline Reflections / parameter & 21 \\
\hline wR2 & 0.1012 \\
\hline R1 & 0.0379 \\
\hline Weights a, b & $0.0546 ; 0.0000$ \\
\hline GoF & 1.058 \\
\hline difference peak / hole $\left(\mathrm{e} \AA^{-3}\right)$ & $1.985(0.118) /-1.871(0.118)$ \\
\hline
\end{tabular}

Note; An highly disorderd half $\mathrm{CH} 2 \mathrm{Cl} 2$ molecule was accounted for using the Platon SQUEEZE function. 
Table S5-2. Atomic Coordinates (A x 10^4) and equivalent isotropic displacement parameters $\left(A^{\wedge} 2 \times 10^{\wedge} 3\right)$ for $6 \mathbf{c}$

\begin{tabular}{|c|c|c|c|c|}
\hline atom & $\mathrm{x}$ & y & $\mathrm{z}$ & $\mathrm{U}(\mathrm{eq})$ \\
\hline Pt (1) & $324(1)$ & $2056(1)$ & $7352(1)$ & 21 (1) \\
\hline $\mathrm{Cl}(1)$ & $-1967(5)$ & $2628(8)$ & $9293(4)$ & $36(1)$ \\
\hline $\operatorname{Br}(1)$ & $-1717(2)$ & $2693(3)$ & $9233(2)$ & $36(1)$ \\
\hline $\mathrm{Cl}(2)$ & $2570(20)$ & $1310(20)$ & $5397(8)$ & $37(1)$ \\
\hline $\operatorname{Br}(2)$ & $2434(4)$ & $1254(5)$ & 5457 (2) & $37(1)$ \\
\hline$P(1)$ & $-863(1)$ & $2698(1)$ & $6697(1)$ & $23(1)$ \\
\hline$P(2)$ & $628(1)$ & $4447(1)$ & $6336(1)$ & $24(1)$ \\
\hline$P(3)$ & $-311(1)$ & $440(1)$ & $7850(1)$ & $23(1)$ \\
\hline$P(4)$ & $1444(1)$ & $494(1)$ & $8528(1)$ & $25(1)$ \\
\hline$N(1)$ & $911(2)$ & $3642(2)$ & $6994(1)$ & $22(1)$ \\
\hline$N(2)$ & $1617(2)$ & $1446(2)$ & $7868(1)$ & $23(1)$ \\
\hline$C(1)$ & $-14(3)$ & $3583(3)$ & $6007(1)$ & $27(1)$ \\
\hline$C(2)$ & $-1392(3)$ & $1832(3)$ & $6309(2)$ & $27(1)$ \\
\hline$C(3)$ & $-2473(3)$ & 1536 (3) & $6553(2)$ & $32(1)$ \\
\hline$C(4)$ & $-2906(3)$ & $907(3)$ & $6252(2)$ & $38(1)$ \\
\hline$C(5)$ & $-2265(3)$ & $574(3)$ & $5697(2)$ & $42(1)$ \\
\hline$C(6)$ & $-1197(3)$ & $866(3)$ & $5458(2)$ & $42(1)$ \\
\hline$C(7)$ & $-737(3)$ & $1486(3)$ & $5756(2)$ & $33(1)$ \\
\hline$C(8)$ & $-2052(2)$ & $3592(3)$ & $7002(1)$ & $25(1)$ \\
\hline$C(9)$ & $-2690(3)$ & $4265(3)$ & $6576(2)$ & $35(1)$ \\
\hline$C(10)$ & $-3558(3)$ & $4997(3)$ & $6806(2)$ & $43(1)$ \\
\hline$C(11)$ & $-3810(3)$ & $5040(3)$ & $7456(2)$ & $44(1)$ \\
\hline$C(12)$ & $-3180(3)$ & $4380(3)$ & $7875(2)$ & 41 (1) \\
\hline$C(13)$ & $-2294(3)$ & $3653(3)$ & $7648(2)$ & $31(1)$ \\
\hline$C(14)$ & $-339(3)$ & $5634(3)$ & $6451(1)$ & $28(1)$ \\
\hline$C(15)$ & $-692(3)$ & $6339(3)$ & $5913(2)$ & $43(1)$ \\
\hline$C(16)$ & $-1440(4)$ & 7255 (3) & $5988(2)$ & 51 (1) \\
\hline$C(17)$ & $-1842(3)$ & $7472(3)$ & $6600(2)$ & $47(1)$ \\
\hline$C(18)$ & $-1497(3)$ & $6780(3)$ & $7135(2)$ & $42(1)$ \\
\hline$C(19)$ & $-758(3)$ & $5858(3)$ & 7067 (2) & $31(1)$ \\
\hline$C(20)$ & $1804(3)$ & $4928(3)$ & $5714(1)$ & $28(1)$ \\
\hline C (21) & $2591(3)$ & $4137(3)$ & $5450(2)$ & $33(1)$ \\
\hline$C(22)$ & 3535 (3) & $4461(3)$ & $4999(2)$ & $39(1)$ \\
\hline$C(23)$ & $3717(3)$ & $5561(4)$ & $4796(2)$ & $43(1)$ \\
\hline$C(24)$ & $2948(3)$ & $6341(3)$ & $5047(2)$ & $42(1)$ \\
\hline$C(25)$ & 1998 (3) & $6028(3)$ & $5508(2)$ & $34(1)$ \\
\hline$C(26)$ & $1419(3)$ & $4059(2)$ & $7404(1)$ & $25(1)$ \\
\hline$C(27)$ & $916(3)$ & $3916(3)$ & $8070(2)$ & $31(1)$ \\
\hline$C(28)$ & 1406 (3) & $4270(3)$ & $8484(2)$ & $38(1)$ \\
\hline C (29) & $2376(3)$ & $4778(3)$ & $8248(2)$ & $43(1)$ \\
\hline$C(30)$ & $2863(3)$ & $4947(3)$ & $7587(2)$ & $40(1)$ \\
\hline$C(31)$ & $2388(3)$ & $4571(3)$ & $7167(2)$ & $31(1)$ \\
\hline$C(32)$ & $-1782(3)$ & $247(3)$ & $8008(1)$ & $26(1)$ \\
\hline$C(33)$ & $-2578(3)$ & $886(3)$ & $8372(2)$ & $32(1)$ \\
\hline$C(34)$ & $-3690(3)$ & $767(3)$ & $8472(2)$ & $38(1)$ \\
\hline$C(35)$ & $-4039(3)$ & $-7(3)$ & $8225(2)$ & $43(1)$ \\
\hline$C(36)$ & $-3257(3)$ & $-668(3)$ & $7887(2)$ & $42(1)$ \\
\hline C (37) & $-2128(3)$ & $-540(3)$ & $7764(2)$ & $35(1)$ \\
\hline C (38) & $427(2)$ & $-701(3)$ & 7505 (2) & $27(1)$ \\
\hline C (39) & $413(3)$ & $-1773(3)$ & $7878(2)$ & $33(1)$ \\
\hline C (40) & $959(3)$ & $-2632(3)$ & $7596(2)$ & $40(1)$ \\
\hline$C(41)$ & $1531(3)$ & $-2411(3)$ & $6943(2)$ & $43(1)$ \\
\hline$C(42)$ & $1548(3)$ & $-1362(3)$ & $6582(2)$ & $40(1)$ \\
\hline$C(43)$ & $997(3)$ & $-491(3)$ & $6861(2)$ & $32(1)$ \\
\hline$C(44)$ & $6(2)$ & $256(3)$ & $8657(1)$ & $26(1)$ \\
\hline C (45) & 2291 (3) & $-781(3)$ & $8462(2)$ & $29(1)$ \\
\hline$C(46)$ & $2182(3)$ & $-1659(3)$ & $9015(2)$ & $37(1)$ \\
\hline$C(47)$ & $2808(3)$ & $-2650(3)$ & $8980(2)$ & $45(1)$ \\
\hline$C(48)$ & $3544(3)$ & $-2790(3)$ & $8414(2)$ & $46(1)$ \\
\hline$C(49)$ & $3644(3)$ & $-1950(3)$ & $7867(2)$ & $45(1)$ \\
\hline$C(50)$ & $3022(3)$ & $-929(3)$ & $7891(2)$ & $36(1)$ \\
\hline$C(51)$ & $1668(3)$ & $846(3)$ & $9239(2)$ & $31(1)$ \\
\hline$C(52)$ & $2601(3)$ & $387(3)$ & $9503(2)$ & $38(1)$ \\
\hline
\end{tabular}




\begin{tabular}{|c|c|c|c|c|}
\hline$C(53)$ & $2804(4)$ & $753(4)$ & $10011(2)$ & $47(1)$ \\
\hline C (54) & $2079(4)$ & $1561(4)$ & $10270(2)$ & $46(1)$ \\
\hline$C(55)$ & $1145(4)$ & $2007(3)$ & $10018(2)$ & $44(1)$ \\
\hline$C(56)$ & $935(3)$ & $1654(3)$ & $9501(2)$ & $36(1)$ \\
\hline C (57) & $2708(2)$ & $1801(3)$ & $7560(2)$ & $26(1)$ \\
\hline C (58) & $3373(3)$ & $2138(3)$ & $7901(2)$ & $29(1)$ \\
\hline C (59) & $4394(3)$ & $2529(3)$ & $7571(2)$ & $37(1)$ \\
\hline$C(60)$ & $4751(3)$ & $2594(3)$ & $6913(2)$ & $41(1)$ \\
\hline$C(61)$ & $4114(3)$ & $2235(3)$ & $6569(2)$ & $39(1)$ \\
\hline$C(62)$ & $3102(3)$ & $1833(3)$ & $6899(2)$ & $30(1)$ \\
\hline$C(63)$ & $5241(4)$ & $1211(5)$ & $4369(2)$ & $69(1)$ \\
\hline $\mathrm{Cl}(3)$ & $4847(8)$ & $810(10)$ & $3766(4)$ & $142(3)$ \\
\hline Cl (4) & $5617(4)$ & $2599(3)$ & $3993(2)$ & $57(1)$ \\
\hline Cl (5) & $5034(8)$ & $604(5)$ & $3821(4)$ & $71(2)$ \\
\hline $\mathrm{Cl}(6)$ & $5686(7)$ & $2370(20)$ & $4280(10)$ & 175 (6) \\
\hline C (64) & $-4659(6)$ & $3017(7)$ & $9757(3)$ & $109(2)$ \\
\hline Cl (7) & $-5605(1)$ & $3353(2)$ & $9238(1)$ & $92(1)$ \\
\hline $\mathrm{Cl}(8)$ & $-5005(3)$ & $8136(3)$ & $9650(2)$ & 231 (2) \\
\hline
\end{tabular}

$\mathrm{U}(\mathrm{eq})$ is defined as $1 / 3$ the trace of the Uij tensor. 
Table S5-3. Bond lengths (A) and angles (deg) for 6c

\begin{tabular}{|c|c|c|c|}
\hline Pt (1) $-\mathrm{N}(2)$ & $2.118(2)$ & Pt $(1)-\mathrm{N}(1)$ & $2.135(2)$ \\
\hline Pt (1) -P (1) & $2.2191(7$ & Pt (1) $-\mathrm{P}(3)$ & $2.2251(8)$ \\
\hline $\mathrm{P}(1)-\mathrm{C}(8)$ & $1.807(3)$ & $P(1)-C(2)$ & $1.812(3)$ \\
\hline$P(1)-C(1)$ & $1.834(3)$ & $\mathrm{P}(2)-\mathrm{N}(1)$ & $1.614(2)$ \\
\hline$P(2)-C(20)$ & $1.795(3)$ & $P(2)-C(14)$ & $1.798(3)$ \\
\hline$P(2)-C(1)$ & $1.808(3)$ & $P(3)-C(38)$ & $1.807(3)$ \\
\hline$P(3)-C(32)$ & $1.817(3)$ & $P(3)-C(44)$ & $1.837(3)$ \\
\hline $\mathrm{P}(4)-\mathrm{N}(2)$ & $1.616(3)$ & $P(4)-C(44)$ & $1.799(3)$ \\
\hline$P(4)-C(45)$ & $1.799(3)$ & $P(4)-C(51)$ & $1.801(3)$ \\
\hline$N(1)-C(26)$ & $1.442(4)$ & $\mathrm{N}(2)-\mathrm{C}(57)$ & $1.443(4)$ \\
\hline$C(2)-C(3)$ & $1.390(5)$ & $C(2)-C(7)$ & $1.402(4)$ \\
\hline$C(3)-C(4)$ & $1.383(5)$ & $C(4)-C(5)$ & $1.391(5)$ \\
\hline$C(5)-C(6)$ & $1.371(6)$ & $C(6)-C(7)$ & $1.389(5)$ \\
\hline$C(8)-C(13)$ & $1.379(4)$ & $C(8)-C(9)$ & $1.400(4)$ \\
\hline$C(9)-C(10)$ & $1.386(4)$ & $C(10)-C(11)$ & $1.380(5)$ \\
\hline$C(11)-C(12)$ & $1.378(5)$ & $C(12)-C(13)$ & $1.393(5)$ \\
\hline$C(14)-C(19)$ & $1.393(4)$ & $C(14)-C(15)$ & $1.399(4)$ \\
\hline$C(15)-C(16)$ & $1.384(5)$ & $C(16)-C(17)$ & $1.378(5)$ \\
\hline$C(17)-C(18)$ & $1.383(5)$ & $C(18)-C(19)$ & $1.382(5)$ \\
\hline$C(20)-C(25)$ & $1.391(5)$ & $C(20)-C(21)$ & $1.410(4)$ \\
\hline$C(21)-C(22)$ & $1.374(5)$ & $C(22)-C(23)$ & $1.388(6)$ \\
\hline$C(23)-C(24)$ & $1.377(6)$ & $C(24)-C(25)$ & $1.386(5)$ \\
\hline$C(26)-C(31)$ & $1.379(5)$ & $C(26)-C(27)$ & $1.404(4)$ \\
\hline$C(27)-C(28)$ & $1.388(4)$ & $C(28)-C(29)$ & $1.378(6)$ \\
\hline$C(29)-C(30)$ & $1.390(5)$ & $C(30)-C(31)$ & $1.402(5)$ \\
\hline$C(32)-C(33)$ & $1.392(4)$ & $C(32)-C(37)$ & $1.400(4)$ \\
\hline$C(33)-C(34)$ & $1.371(5)$ & $C(34)-C(35)$ & $1.392(5)$ \\
\hline$C(35)-C(36)$ & $1.373(5)$ & $C(36)-C(37)$ & $1.389(5)$ \\
\hline$C(38)-C(43)$ & $1.383(4)$ & $C(38)-C(39)$ & $1.395(5)$ \\
\hline$C(39)-C(40)$ & $1.382(5)$ & $C(40)-C(41)$ & $1.401(5)$ \\
\hline$C(41)-C(42)$ & $1.363(5)$ & $C(42)-C(43)$ & $1.393(5)$ \\
\hline$C(45)-C(50)$ & $1.380(5)$ & $C(45)-C(46)$ & $1.413(5)$ \\
\hline$C(46)-C(47)$ & $1.376(5)$ & $C(47)-C(48)$ & $1.372(6)$ \\
\hline$C(48)-C(49)$ & $1.375(6)$ & $C(49)-C(50)$ & $1.403(5)$ \\
\hline$C(51)-C(56)$ & $1.392(5)$ & $C(51)-C(52)$ & $1.397(5)$ \\
\hline$C(52)-C(53)$ & $1.384(5)$ & $C(53)-C(54)$ & $1.385(6)$ \\
\hline$C(54)-C(55)$ & $1.382(6)$ & $C(55)-C(56)$ & $1.397(5)$ \\
\hline$C(57)-C(62)$ & $1.389(4)$ & $C(57)-C(58)$ & $1.403(4)$ \\
\hline$C(58)-C(59)$ & $1.392(5)$ & $C(59)-C(60)$ & $1.372(5)$ \\
\hline$C(60)-C(61)$ & $1.398(5)$ & $C(61)-C(62)$ & $1.388(5)$ \\
\hline$C(63)-C l(6)$ & $1.59(1)$ & $C(63)-C l(5)$ & $1.652(9)$ \\
\hline$C(63)-C l(3)$ & $1.712(8)$ & $C(63)-C l(4)$ & $1.822(7)$ \\
\hline $\mathrm{C}(64)-\mathrm{Cl}(8) \# 2$ & $1.716(8)$ & $C(64)-C l(7)$ & 1.755 (6) \\
\hline
\end{tabular}

$\mathrm{N}(2)-\mathrm{Pt}(1)-\mathrm{N}(1)$

$\mathrm{N}$ (1) -Pt (1) -P (1)

$\mathrm{N}(1)-\mathrm{Pt}(1)-\mathrm{P}(3)$

$\mathrm{C}(8)-\mathrm{P}(1)-\mathrm{C}(2)$

$C(2)-P(1)-C(1)$

$\mathrm{C}(2)-\mathrm{P}(1)-\mathrm{Pt}(1)$

$\mathrm{N}(1)-\mathrm{P}(2)-\mathrm{C}(20)$

$\mathrm{C}(20)-\mathrm{P}(2)-\mathrm{C}(14)$

$\mathrm{C}(20)-\mathrm{P}(2)-\mathrm{C}(1)$

$\mathrm{C}(38)-\mathrm{P}(3)-\mathrm{C}(32)$

$\mathrm{C}(32)-\mathrm{P}(3)-\mathrm{C}(44)$

$\mathrm{C}(32)-\mathrm{P}(3)-\mathrm{Pt}(1)$

$\mathrm{N}(2)-\mathrm{P}(4)-\mathrm{C}(44)$

$\mathrm{C}(44)-\mathrm{P}(4)-\mathrm{C}(45)$

$\mathrm{C}(44)-\mathrm{P}(4)-\mathrm{C}(51)$

$\mathrm{C}(26)-\mathrm{N}(1)-\mathrm{P}(2)$

$\mathrm{P}(2)-\mathrm{N}(1)-\mathrm{Pt}(1)$

$\mathrm{C}(57)-\mathrm{N}(2)-\mathrm{Pt}(1)$

$\mathrm{P}(2)-\mathrm{C}(1)-\mathrm{P}(1)$

$\mathrm{C}(3)-\mathrm{C}(2)-\mathrm{P}(1)$

$C(4)-C(3)-C(2)$

$C(6)-C(5)-C(4)$
$92.9(1)$

$84.55(7)$

$172.80(6)$

$106.1(1)$

$103.2(1)$

$122.9(1)$

$116.2(1)$

$106.7(2)$

$106.5(1)$

$105.3(1)$

$105.3(1)$

$121.8(1)$

$103.1(1)$

$107.9(2)$

$109.4(2)$

$119.4(2)$

$121.5(1)$

$117.0(2)$

$107.6(1)$

$119.4(2)$

$120.4(3)$

$119.3(3)$
$\mathrm{N}(2)-\mathrm{Pt}(1)-\mathrm{P}(1)$

$\mathrm{N}(2)-\mathrm{Pt}(1)-\mathrm{P}(3)$

$\mathrm{P}(1)-\mathrm{Pt}(1)-\mathrm{P}(3)$

$\mathrm{C}(8)-\mathrm{P}(1)-\mathrm{C}(1)$

$\mathrm{C}(8)-\mathrm{P}(1)-\mathrm{Pt}(1)$

$\mathrm{C}(1)-\mathrm{P}(1)-\mathrm{Pt}(1)$

$\mathrm{N}(1)-\mathrm{P}(2)-\mathrm{C}(14)$

$\mathrm{N}(1)-\mathrm{P}(2)-\mathrm{C}(1)$

$\mathrm{C}(14)-\mathrm{P}(2)-\mathrm{C}(1)$

$\mathrm{C}(38)-\mathrm{P}(3)-\mathrm{C}(44)$

$\mathrm{C}(38)-\mathrm{P}(3)-\mathrm{Pt}(1)$

$\mathrm{C}(44)-\mathrm{P}(3)-\mathrm{Pt}(1)$

$\mathrm{N}(2)-\mathrm{P}(4)-\mathrm{C}(45)$

$\mathrm{N}(2)-\mathrm{P}(4)-\mathrm{C}(51)$

$\mathrm{C}(45)-\mathrm{P}(4)-\mathrm{C}(51)$

$\mathrm{C}(26)-\mathrm{N}(1)-\mathrm{Pt}(1)$

$\mathrm{C}(57)-\mathrm{N}(2)-\mathrm{P}(4)$

$\mathrm{P}(4)-\mathrm{N}(2)-\mathrm{Pt}(1)$

$\mathrm{C}(3)-\mathrm{C}(2)-\mathrm{C}(7)$

$\mathrm{C}(7)-\mathrm{C}(2)-\mathrm{P}(1)$

C (3) $-\mathrm{C}(4)-\mathrm{C}(5)$

$C(5)-C(6)-C(7)$
$172.76(7)$

$83.47(7)$

$99.75(3)$

$105.3(2)$

$115.3(1)$

$101.8(1)$

$115.5(1)$

$103.2(1)$

$108.2(2)$

$107.4(1)$

$114.8(1)$

$100.9(1)$

$114.7(1)$

$115.5(2)$

$106.0(2)$

$118.4(2)$

$120.6(2)$

$121.8(1)$

$119.7(3)$

$120.9(2)$

$120.2(3)$

$121.8(3)$ 
$C(13)-C(8)-C(9)$

$C(9)-C(8)-P(1)$

$C(11)-C(10)-C(9)$

$C(11)-C(12)-C(13)$

$C(19)-C(14)-C(15)$

$\mathrm{C}(15)-\mathrm{C}(14)-\mathrm{P}(2)$

$\mathrm{C}(17)-\mathrm{C}(16)-\mathrm{C}(15)$

C (19) -C (18)-C (17)

$C(25)-C(20)-C(21)$

$\mathrm{C}(21)-\mathrm{C}(20)-\mathrm{P}(2)$

$\mathrm{C}(21)-\mathrm{C}(22)-\mathrm{C}(23)$

$C(23)-C(24)-C(25)$

$C(31)-C(26)-C(27)$

$\mathrm{C}(27)-\mathrm{C}(26)-\mathrm{N}(1)$

$\mathrm{C}(29)-\mathrm{C}(28)-\mathrm{C}(27)$

$C(29)-C(30)-C(31)$

$C(33)-C(32)-C(37)$

$\mathrm{C}(37)-\mathrm{C}(32)-\mathrm{P}(3)$

$\mathrm{C}(33)-\mathrm{C}(34)-\mathrm{C}(35)$

$C(35)-C(36)-C(37)$

$C(43)-C(38)-C(39)$

$\mathrm{C}(39)-\mathrm{C}(38)-\mathrm{P}(3)$

$C(39)-C(40)-C(41)$

$C(41)-C(42)-C(43)$

$\mathrm{P}(4)-\mathrm{C}(44)-\mathrm{P}(3)$

$\mathrm{C}(50)-\mathrm{C}(45)-\mathrm{P}(4)$

$C(47)-C(46)-C(45)$

C (47) $-\mathrm{C}(48)-\mathrm{C}(49)$

$C(45)-C(50)-C(49)$

$C(56)-C(51)-P(4)$

$C(53)-C(52)-C(51)$

$C(55)-C(54)-C(53)$

$C(51)-C(56)-C(55)$

$\mathrm{C}(62)-\mathrm{C}(57)-\mathrm{N}(2)$

$\mathrm{C}(59)-\mathrm{C}(58)-\mathrm{C}(57)$

$C(59)-C(60)-C(61)$

$C(61)-C(62)-C(57)$

$\mathrm{Cl}(6)-\mathrm{C}(63)-\mathrm{Cl}(3)$

$\mathrm{Cl}(6)-\mathrm{C}(63)-\mathrm{Cl}(4)$

$\mathrm{Cl}(3)-\mathrm{C}(63)-\mathrm{Cl}(4)$
$119.9(3)$

$120.1(2)$

$120.1(3)$

$120.3(3)$

$119.2(3)$

$119.5(2)$

$119.5(3)$

$120.9(3)$

$119.1(3)$

$117.7(3)$

$120.5(3)$

$120.2(4)$

$119.4(3)$

$118.4(3)$

$120.9(3)$

$120.1(4)$

$119.3(3)$

$120.5(2)$

$120.7(3)$

$120.7(3)$

$120.5(3)$

$120.9(2)$

$119.5(4)$

$120.1(3)$

$107.2(2)$

$122.3(3)$

$119.7(3)$

$120.4(4)$

$119.6(3)$

$118.8(2)$

$119.9(4)$

$119.8(3)$

$119.9(3)$

$118.6(3)$

$119.9(3)$

$120.6(3)$

$121.0(3)$

$124(1)$

$20(1)$

$103.9(5)$ 
Table S5-4. Anisotropic displacement parameters (A^2 x 10^3) for

\begin{tabular}{|c|c|c|c|c|c|c|}
\hline atom & U11 & $\mathrm{U} 22$ & U33 & U23 & U13 & U12 \\
\hline Pt (1) & $24(1)$ & $20(1)$ & 21 (1) & $-4(1)$ & $-7(1)$ & $-2(1)$ \\
\hline $\mathrm{Cl}(1)$ & 35 (1) & $44(1)$ & $30(1)$ & $-8(1)$ & $-7(1)$ & $-2(1)$ \\
\hline $\operatorname{Br}(1)$ & $35(1)$ & $44(1)$ & $30(1)$ & $-8(1)$ & $-7(1)$ & $-2(1)$ \\
\hline $\mathrm{Cl}(2)$ & $48(1)$ & $39(1)$ & $25(1)$ & $-6(1)$ & $-6(1)$ & $-12(1)$ \\
\hline $\operatorname{Br}(2)$ & $48(1)$ & $39(1)$ & $25(1)$ & $-6(1)$ & $-6(1)$ & $-12(1)$ \\
\hline P (1) & $25(1)$ & $24(1)$ & $22(1)$ & $-6(1)$ & $-7(1)$ & $-1(1)$ \\
\hline$P(2)$ & $28(1)$ & $21(1)$ & 21 (1) & $-4(1)$ & $-6(1)$ & -1 (1) \\
\hline$P(3)$ & $26(1)$ & $20(1)$ & $25(1)$ & $-4(1)$ & $-7(1)$ & $-3(1)$ \\
\hline$P(4)$ & $29(1)$ & $23(1)$ & $23(1)$ & $-3(1)$ & $-9(1)$ & $-3(1)$ \\
\hline$N(1)$ & $28(1)$ & $14(1)$ & $25(1)$ & $-3(1)$ & $-8(1)$ & $-5(1)$ \\
\hline $\mathrm{N}(2)$ & $24(1)$ & $22(1)$ & $25(1)$ & $-2(1)$ & $-10(1)$ & $-2(1)$ \\
\hline$C(1)$ & $33(2)$ & $26(2)$ & $22(1)$ & $-7(1)$ & $-9(1)$ & -1 (1) \\
\hline$C(2)$ & $28(2)$ & $25(2)$ & $31(2)$ & $-9(1)$ & $-13(1)$ & $0(1)$ \\
\hline$C(3)$ & $32(2)$ & $34(2)$ & $33(2)$ & $-10(1)$ & $-9(1)$ & $-6(1)$ \\
\hline C (4) & $38(2)$ & $38(2)$ & $45(2)$ & $-13(2)$ & $-15(2)$ & $-8(2)$ \\
\hline$C(5)$ & $55(2)$ & $31(2)$ & $51(2)$ & $-18(2)$ & $-24(2)$ & $1(2)$ \\
\hline$C(6)$ & $42(2)$ & $43(2)$ & $50(2)$ & $-29(2)$ & $-18(2)$ & $9(2)$ \\
\hline$C(7)$ & $31(2)$ & $36(2)$ & $37(2)$ & $-17(2)$ & $-8(1)$ & $0(1)$ \\
\hline$C(8)$ & $23(1)$ & $24(2)$ & $28(1)$ & $-9(1)$ & $-5(1)$ & $-1(1)$ \\
\hline$C(9)$ & $31(2)$ & $42(2)$ & $34(2)$ & $-14(2)$ & $-14(1)$ & $6(2)$ \\
\hline$C(10)$ & $35(2)$ & $44(2)$ & $52(2)$ & $-14(2)$ & $-17(2)$ & $10(2)$ \\
\hline$C(11)$ & $34(2)$ & $43(2)$ & $55(2)$ & $-21(2)$ & $-5(2)$ & $8(2)$ \\
\hline$C(12)$ & $43(2)$ & $45(2)$ & $32(2)$ & $-15(2)$ & $2(2)$ & $-2(2)$ \\
\hline$C(13)$ & $33(2)$ & $30(2)$ & $30(2)$ & $-9(1)$ & $-5(1)$ & $-2(1)$ \\
\hline$C(14)$ & $33(2)$ & $25(2)$ & $25(1)$ & $-3(1)$ & $-8(1)$ & $1(1)$ \\
\hline$C(15)$ & $58(2)$ & $39(2)$ & $28(2)$ & $-5(2)$ & $-13(2)$ & $12(2)$ \\
\hline$C(16)$ & $65(3)$ & $40(2)$ & $38(2)$ & $0(2)$ & $-17(2)$ & $17(2)$ \\
\hline$C(17)$ & $54(2)$ & $31(2)$ & $51(2)$ & $-12(2)$ & $-9(2)$ & $14(2)$ \\
\hline$C(18)$ & $51(2)$ & $37(2)$ & $37(2)$ & $-14(2)$ & $-6(2)$ & $3(2)$ \\
\hline C (19) & $36(2)$ & $28(2)$ & $28(2)$ & $-7(1)$ & $-8(1)$ & $-2(1)$ \\
\hline$C(20)$ & $34(2)$ & $28(2)$ & $23(1)$ & $-5(1)$ & $-7(1)$ & $-5(1)$ \\
\hline C (21) & $34(2)$ & $31(2)$ & $33(2)$ & $-6(1)$ & $-8(1)$ & $-3(1)$ \\
\hline$C(22)$ & $29(2)$ & $49(2)$ & $36(2)$ & $-7(2)$ & $-5(1)$ & $-1(2)$ \\
\hline$C(23)$ & $34(2)$ & $62(3)$ & $29(2)$ & $3(2)$ & $-7(1)$ & $-19(2)$ \\
\hline$C(24)$ & $51(2)$ & $40(2)$ & $36(2)$ & $8(2)$ & $-17(2)$ & $-19(2)$ \\
\hline$C(25)$ & $42(2)$ & $29(2)$ & $32(2)$ & $-5(1)$ & $-10(1)$ & $-6(2)$ \\
\hline$C(26)$ & $32(2)$ & $18(2)$ & $27(1)$ & $-6(1)$ & $-12(1)$ & $2(1)$ \\
\hline$C(27)$ & $40(2)$ & $24(2)$ & $28(2)$ & $-3(1)$ & $-12(1)$ & $-2(1)$ \\
\hline$C(28)$ & $60(2)$ & $27(2)$ & $29(2)$ & $-8(1)$ & $-17(2)$ & $1(2)$ \\
\hline C (29) & $65(3)$ & $24(2)$ & $54(2)$ & $-9(2)$ & $-39(2)$ & $-1(2)$ \\
\hline$C(30)$ & $43(2)$ & $28(2)$ & $57(2)$ & $-6(2)$ & $-26(2)$ & $-5(2)$ \\
\hline$C(31)$ & $35(2)$ & $23(2)$ & $38(2)$ & $-2(1)$ & $-16(1)$ & $-4(1)$ \\
\hline$C(32)$ & $29(2)$ & $21(2)$ & $27(1)$ & $-4(1)$ & $-6(1)$ & $-4(1)$ \\
\hline$C(33)$ & $32(2)$ & $34(2)$ & $29(2)$ & $-6(1)$ & $-7(1)$ & $-5(1)$ \\
\hline$C(34)$ & $35(2)$ & $39(2)$ & $35(2)$ & $-6(2)$ & $-3(1)$ & $3(2)$ \\
\hline$C(35)$ & $31(2)$ & $45(2)$ & $49(2)$ & $-3(2)$ & $-9(2)$ & $-7(2)$ \\
\hline$C(36)$ & $36(2)$ & $38(2)$ & $58(2)$ & $-14(2)$ & $-12(2)$ & $-13(2)$ \\
\hline C (37) & $31(2)$ & $32(2)$ & $47(2)$ & $-13(2)$ & $-10(1)$ & $-5(1)$ \\
\hline C (38) & $25(1)$ & $22(2)$ & $34(2)$ & $-9(1)$ & $-8(1)$ & $0(1)$ \\
\hline C (39) & $34(2)$ & $29(2)$ & $35(2)$ & $-7(1)$ & $-6(1)$ & $-4(1)$ \\
\hline$C(40)$ & $42(2)$ & $23(2)$ & $54(2)$ & $-6(2)$ & $-13(2)$ & $-1(2)$ \\
\hline C (41) & $48(2)$ & $27(2)$ & $55(2)$ & $-18(2)$ & $-13(2)$ & $6(2)$ \\
\hline$C(42)$ & $44(2)$ & $35(2)$ & $37(2)$ & $-11(2)$ & $-4(2)$ & $2(2)$ \\
\hline$C(43)$ & $35(2)$ & $29(2)$ & $30(2)$ & $-7(1)$ & $-9(1)$ & $1(1)$ \\
\hline$C(44)$ & $29(2)$ & $27(2)$ & $23(1)$ & $-3(1)$ & $-6(1)$ & $-6(1)$ \\
\hline C (45) & $31(2)$ & $24(2)$ & $32(2)$ & $-4(1)$ & $-13(1)$ & $-1(1)$ \\
\hline$C(46)$ & $42(2)$ & $29(2)$ & $37(2)$ & $-1(1)$ & $-11(2)$ & $-2(2)$ \\
\hline$C(47)$ & $56(2)$ & $29(2)$ & $50(2)$ & $1(2)$ & $-24(2)$ & $-2(2)$ \\
\hline$C(48)$ & $46(2)$ & $28(2)$ & $64(3)$ & $-12(2)$ & $-16(2)$ & $5(2)$ \\
\hline$C(49)$ & $43(2)$ & $35(2)$ & $53(2)$ & $-11(2)$ & $-6(2)$ & $2(2)$ \\
\hline$C(50)$ & $37(2)$ & $28(2)$ & $42(2)$ & $-6(2)$ & $-11(2)$ & $-4(1)$ \\
\hline$C(51)$ & $39(2)$ & $28(2)$ & $27(1)$ & $-2(1)$ & $-10(1)$ & $-10(1)$ \\
\hline C (52) & $49(2)$ & $37(2)$ & $34(2)$ & $-2(2)$ & $-18(2)$ & $-9(2)$ \\
\hline C (53) & $60(3)$ & $47(3)$ & $40(2)$ & $2(2)$ & $-29(2)$ & $-13(2)$ \\
\hline
\end{tabular}




\begin{tabular}{|c|c|c|c|c|c|c|}
\hline C (54) & $67(3)$ & $49(3)$ & $28(2)$ & $-1(2)$ & $-14(2)$ & $-29(2)$ \\
\hline$C(55)$ & $66(3)$ & $39(2)$ & $29(2)$ & $-8(2)$ & $-7(2)$ & $-17(2)$ \\
\hline$C(56)$ & $47(2)$ & $33(2)$ & $26(2)$ & $-3(1)$ & $-8(1)$ & $-10(2)$ \\
\hline$C(57)$ & $24(1)$ & $19(2)$ & $34(2)$ & $-2(1)$ & $-11(1)$ & $1(1)$ \\
\hline$C(58)$ & $29(2)$ & $25(2)$ & $36(2)$ & $-2(1)$ & $-16(1)$ & $-2(1)$ \\
\hline$C(59)$ & $34(2)$ & $30(2)$ & $50(2)$ & $1(2)$ & $-20(2)$ & $-7(1)$ \\
\hline$C(60)$ & $25(2)$ & $38(2)$ & $52(2)$ & $2(2)$ & $-7(2)$ & $-6(2)$ \\
\hline$C(61)$ & $33(2)$ & $35(2)$ & $40(2)$ & $-2(2)$ & $-3(1)$ & $2(2)$ \\
\hline$C(62)$ & $30(2)$ & $28(2)$ & $31(2)$ & $-4(1)$ & $-7(1)$ & $0(1)$ \\
\hline$C(63)$ & $68(3)$ & $74(4)$ & $69(3)$ & $-25(3)$ & $-22(3)$ & $2(3)$ \\
\hline Cl (3) & $127(4)$ & 247 (8) & $87(3)$ & $-60(4)$ & $5(3)$ & $-128(5)$ \\
\hline Cl (4) & $52(2)$ & $44(2)$ & $77(2)$ & $-28(2)$ & $0(1)$ & $-10(1)$ \\
\hline Cl (5) & $97(4)$ & $51(3)$ & $72(3)$ & $-38(2)$ & $11(3)$ & $-36(2)$ \\
\hline Cl (6) & $53(3)$ & $200(10)$ & $350(20)$ & $-220(10)$ & $-23(7)$ & $-5(5)$ \\
\hline$C(64)$ & $84(4)$ & $133(7)$ & 105 (5) & $-2(5)$ & $-33(4)$ & $-21(4)$ \\
\hline $\mathrm{Cl}(7)$ & $67(1)$ & $130(2)$ & 70 (1) & $-13(1)$ & $-10(1)$ & $-6(1)$ \\
\hline $\mathrm{Cl}(8)$ & $173(3)$ & $273(4)$ & $243(3)$ & $112(3)$ & $-140(3)$ & $-130(3)$ \\
\hline
\end{tabular}

The anisotropic displacement factor exponent takes the form $2 \mathrm{pi}^{\wedge} 2\left[\mathrm{~h}^{\wedge} 2 \mathrm{a}^{\star \wedge} 2 \mathrm{U}(11)+\ldots+2 \mathrm{hka}{ }^{\star} \mathrm{b} * \mathrm{U}(12)\right]$ 
Table S5-5. Hydrogen Coordinates (A x 10^4) and equivalent isotropic displacement parameters $\left(A^{\wedge} 2 \times 10^{\wedge} 3\right)$ for $6 \mathbf{c}$

\begin{tabular}{|c|c|c|c|c|}
\hline atom & $\mathrm{x}$ & y & $\mathrm{z}$ & $\mathrm{U}(\mathrm{eq})$ \\
\hline $\mathrm{H}(1 \mathrm{~A})$ & 567 & 3132 & 5755 & 32 \\
\hline $\mathrm{H}(1 \mathrm{~B})$ & -489 & 4040 & 5714 & 32 \\
\hline $\mathrm{H}(3)$ & -2918 & 1767 & 6929 & 38 \\
\hline $\mathrm{H}(4)$ & -3643.0002 & 700 & 6424 & 46 \\
\hline $\mathrm{H}(5)$ & -2563 & 150 & 5486 & 51 \\
\hline $\mathrm{H}(6)$ & -762 & 638 & 5079 & 50 \\
\hline $\mathrm{H}(7)$ & 8 & 1672 & 5587 & 40 \\
\hline $\mathrm{H}(9)$ & -2530 & 4220 & 6131 & 41 \\
\hline $\mathrm{H}(10)$ & -3978.9998 & 5470 & 6516 & 52 \\
\hline $\mathrm{H}(11)$ & -4420 & 5526 & 7616 & 53 \\
\hline $\mathrm{H}(12)$ & -3349 & 4422 & 8320 & 49 \\
\hline $\mathrm{H}(13)$ & -1860 & 3201 & 7937 & 37 \\
\hline $\mathrm{H}(15)$ & -416 & 6187 & 5491 & 52 \\
\hline $\mathrm{H}(16)$ & -1676 & 7731 & 5621 & 61 \\
\hline $\mathrm{H}(17)$ & -2357 & 8098 & 6655 & 57 \\
\hline $\mathrm{H}(18)$ & -1771 & 6942 & 7554 & 50 \\
\hline $\mathrm{H}(19)$ & -538 & 5378 & 7439 & 37 \\
\hline $\mathrm{H}(21)$ & 2469 & 3383 & 5585 & 39 \\
\hline $\mathrm{H}(22)$ & 4066 & 3926 & 4824 & 47 \\
\hline $\mathrm{H}(23)$ & 4373 & 5777 & 4484 & 51 \\
\hline $\mathrm{H}(24)$ & 3070 & 7094 & 4903 & 51 \\
\hline $\mathrm{H}(25)$ & 1476 & 6567 & 5685 & 41 \\
\hline $\mathrm{H}(27)$ & 241 & 3577 & 8237 & 37 \\
\hline $\mathrm{H}(28)$ & 1069 & 4161 & 8935 & 45 \\
\hline $\mathrm{H}(29)$ & 2710 & 5011 & 8536 & 52 \\
\hline $\mathrm{H}(30)$ & 3518 & 5317 & 7419 & 48 \\
\hline $\mathrm{H}(31)$ & 2735 & 4669 & 6717 & 38 \\
\hline $\mathrm{H}(33)$ & -2349 & 1405 & 8551 & 38 \\
\hline $\mathrm{H}(34)$ & -4229 & 1218 & 8712 & 46 \\
\hline $\mathrm{H}(35)$ & -4812 & -77 & 8291 & 51 \\
\hline $\mathrm{H}(36)$ & -3491 & -1219 & 7735 & 50 \\
\hline $\mathrm{H}(37)$ & -1593 & -983 & 7516 & 42 \\
\hline H (39) & 30 & -1912 & 8322 & 40 \\
\hline $\mathrm{H}(40)$ & 946 & -3366 & 7843 & 48 \\
\hline $\mathrm{H}(41)$ & 1912 & -3000 & 6749 & 51 \\
\hline $\mathrm{H}(42)$ & 1936 & -1224 & 6138 & 48 \\
\hline $\mathrm{H}(43)$ & 1012 & 241 & 6610 & 38 \\
\hline $\mathrm{H}(44 \mathrm{~A})$ & -486 & 783 & 8898 & 31 \\
\hline $\mathrm{H}(44 \mathrm{~B})$ & -113 & -498 & 8914 & 31 \\
\hline $\mathrm{H}(46)$ & 1679 & -1566 & 9410 & 44 \\
\hline $\mathrm{H}(47)$ & 2728 & -3243 & 9351 & 54 \\
\hline $\mathrm{H}(48)$ & 3987 & -3471 & 8399 & 55 \\
\hline $\mathrm{H}(49)$ & 4136 & -2061 & 7472 & 54 \\
\hline $\mathrm{H}(50)$ & 3103 & -343 & 7516 & 43 \\
\hline $\mathrm{H}(52)$ & 3096 & -176 & 9333 & 46 \\
\hline $\mathrm{H}(53)$ & 3446 & 447 & 10183 & 56 \\
\hline $\mathrm{H}(54)$ & 2223 & 1807 & 10619 & 55 \\
\hline $\mathrm{H}(55)$ & 643 & 2556 & 10197 & 52 \\
\hline H ( 56$)$ & 295 & 1965 & 9328 & 43 \\
\hline $\mathrm{H}(58)$ & 3125 & 2099 & 8356 & 35 \\
\hline $\mathrm{H}(59)$ & 4846 & 2751 & 7802 & 45 \\
\hline $\mathrm{H}(60)$ & 5437 & 2885 & 6689 & 49 \\
\hline $\mathrm{H}(61)$ & 4371 & 2266 & 6115 & 46 \\
\hline $\mathrm{H}(62)$ & 2673 & 1576 & 6669 & 36 \\
\hline $\mathrm{H}(63 \mathrm{~A})$ & 5887 & 724 & 4505 & 82 \\
\hline $\mathrm{H}(63 \mathrm{~B})$ & 4617 & 1208 & 4754 & 82 \\
\hline $\mathrm{H}(63 \mathrm{C})$ & 4513 & 1241 & 4682 & 82 \\
\hline $\mathrm{H}(63 \mathrm{D})$ & 5751 & 673 & 4607 & 82 \\
\hline $\mathrm{H}(64 \mathrm{~A})$ & -3893 & 2885 & 9500 & 130 \\
\hline $\mathrm{H}(64 \mathrm{~B})$ & -4677 & 3637 & 9963 & 130 \\
\hline
\end{tabular}


Figure S6: Molecular structure of $\mathbf{6 e}$

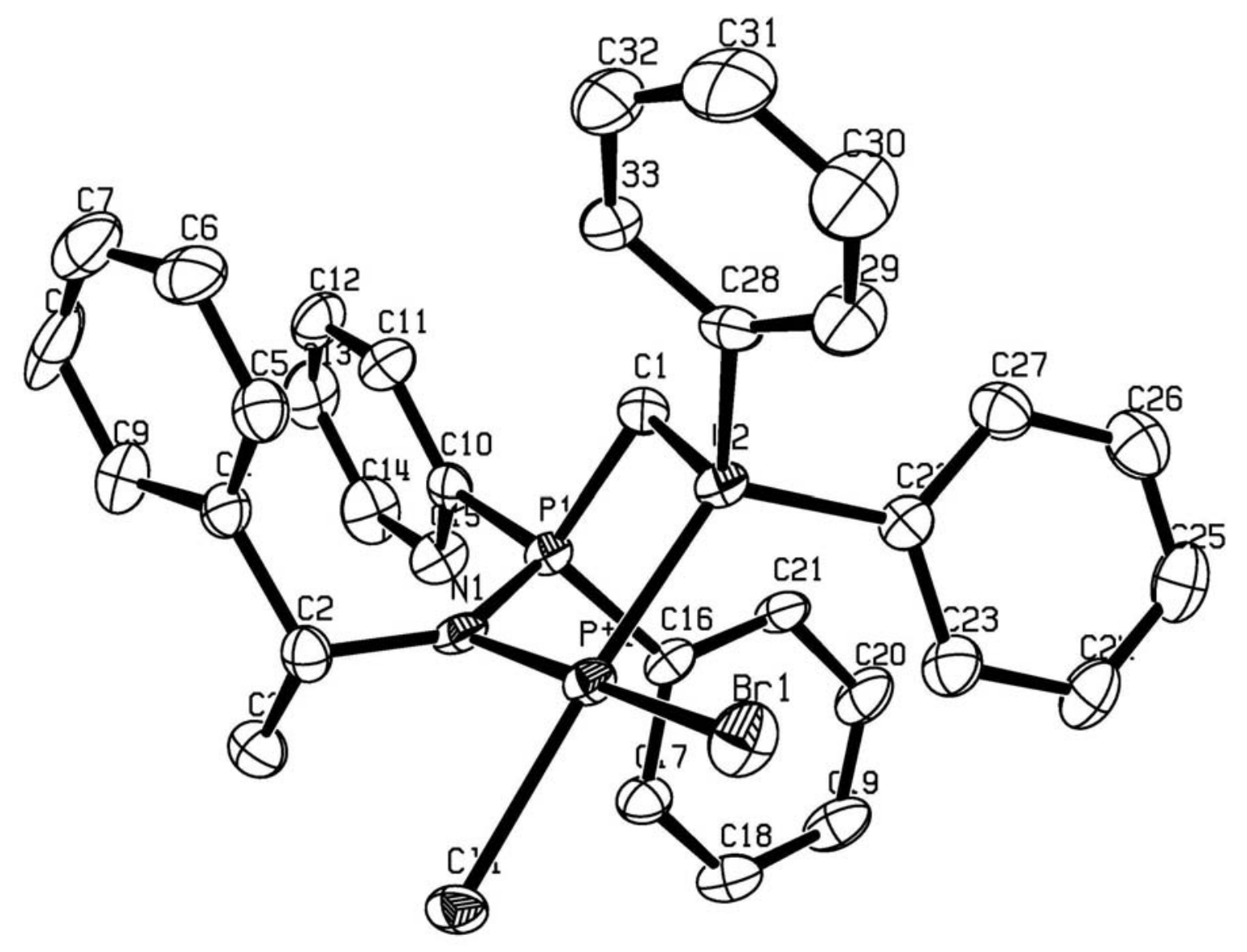


Table S6-1. Crystal data for $6 \mathbf{e}$

\begin{tabular}{|c|c|}
\hline Compound & $6 e$ \\
\hline Molecular formula & $\mathrm{C}_{33} \mathrm{H}_{31} \mathrm{BrClNP}_{2} \mathrm{Pt}, \mathrm{C}_{4} \mathrm{H}_{8} \mathrm{O}, \mathrm{CH}_{2} \mathrm{Cl}_{2}$ \\
\hline Molecular weight & 971.01 \\
\hline Crystal habit & yellow plate \\
\hline Crystal dimensions $(\mathrm{mm})$ & $0.20 \times 0.18 \times 0.05$ \\
\hline Crystal system & monoclinic \\
\hline Space group & $\mathrm{P} 2{ }_{1}$ \\
\hline $\mathrm{a}(\AA)$ & $8.5010(10)$ \\
\hline $\mathrm{b}(\AA)$ & $16.3370(10)$ \\
\hline$c(\AA)$ & $13.5450(10)$ \\
\hline$\alpha\left(^{\circ}\right)$ & 90.00 \\
\hline$\beta\left(\left(^{\circ}\right)\right.$ & $94.0500(10)$ \\
\hline$\gamma\left({ }^{\circ}\right)$ & 90.00 \\
\hline $\mathrm{V}\left(\AA^{3}\right)$ & $1876.4(3)$ \\
\hline Z & 2 \\
\hline$d\left(g-\mathrm{cm}^{-3}\right)$ & 1.719 \\
\hline F000 & 956 \\
\hline$\mu\left(\mathrm{cm}^{-1}\right)$ & 5.134 \\
\hline Absorption corrections & multi-scan ; $0.4266 \min , 0.7834 \max$ \\
\hline Diffractometer & KappaCCD \\
\hline X-ray source & $\mathrm{MoK} \alpha$ \\
\hline$\lambda(\AA)$ & 0.71069 \\
\hline Monochromator & graphite \\
\hline $\mathrm{T}(\mathrm{K})$ & $150.0(1)$ \\
\hline Scan mode & phi and omega scans \\
\hline Maximum $\theta$ & 30.02 \\
\hline HKL ranges & $-1111 ;-2220 ;-1919$ \\
\hline Reflections measured & 10110 \\
\hline Unique data & 10110 \\
\hline Rint & 0.0000 \\
\hline Reflections used & 9116 \\
\hline Criterion & $>2 \operatorname{sigma}(\mathrm{I})$ \\
\hline Refinement type & Fsqd \\
\hline Hydrogen atoms & mixed \\
\hline Parameters refined & 400 \\
\hline Reflections / parameter & 22 \\
\hline wR2 & 0.1001 \\
\hline R1 & 0.0390 \\
\hline Flack's parameter & $0.016(5)$ \\
\hline Weights $a, b$ & $0.0641 ; 0.0000$ \\
\hline GoF & 1.005 \\
\hline difference peak / hole $\left(\mathrm{e} \AA^{-3}\right)$ & $1.405(0.157) /-2.506(0.157)$ \\
\hline
\end{tabular}


Table S6-2. Atomic Coordinates (A x 10^4) and equivalent isotropic displacement parameters $\left(A^{\wedge} 2 \times 10^{\wedge} 3\right)$ for $6 e$

\begin{tabular}{|c|c|c|c|c|}
\hline atom & $\mathrm{x}$ & $\mathrm{y}$ & z & $\mathrm{U}(\mathrm{eq})$ \\
\hline Pt (1) & $-218(1)$ & $2014(1)$ & 421 (1) & $20(1)$ \\
\hline $\mathrm{Cl}(1)$ & $-2637(1)$ & $1632(1)$ & $-423(1)$ & $26(1)$ \\
\hline $\operatorname{Br}(1)$ & $-1542(1)$ & $2090(1)$ & $1959(1)$ & $35(1)$ \\
\hline$P(1)$ & $2639(2)$ & $1739(1)$ & $-774(1)$ & $20(1)$ \\
\hline$P(2)$ & $2085(2)$ & $2262(1)$ & $1212(1)$ & $21(1)$ \\
\hline $\mathrm{N}(1)$ & $843(4)$ & $2032(5)$ & $-925(3)$ & $22(1)$ \\
\hline$C(1)$ & $3557(6)$ & $2291(4)$ & $278(4)$ & $24(1)$ \\
\hline$C(2)$ & $7(6)$ & $2432(4)$ & $-1769(4)$ & $25(1)$ \\
\hline$C(3)$ & $-230(7)$ & $1858(4)$ & $-2657(4)$ & $33(2)$ \\
\hline$C(4)$ & $761(6)$ & $3265(4)$ & $-2007(4)$ & $27(1)$ \\
\hline$C(5)$ & $951(7)$ & $3835(4)$ & $-1242(4)$ & $30(1)$ \\
\hline$C(6)$ & $1606(8)$ & $4598(4)$ & $-1391(6)$ & $41(2)$ \\
\hline$C(7)$ & $2080(8)$ & $4795(5)$ & $-2324(7)$ & $46(2)$ \\
\hline$C(8)$ & $1890(10)$ & $4246(5)$ & $-3095(7)$ & $56(2)$ \\
\hline$C(9)$ & $1218(8)$ & $3462(4)$ & $-2951(5)$ & $35(1)$ \\
\hline$C(10)$ & $3774(5)$ & $1912(5)$ & $-1829(4)$ & $22(1)$ \\
\hline$C(11)$ & $4441(7)$ & $2677(4)$ & $-1982(5)$ & $32(1)$ \\
\hline$C(12)$ & $5329(7)$ & $2794(4)$ & $-2801(5)$ & $37(2)$ \\
\hline$C(13)$ & $5520(7)$ & $2172(6)$ & $-3455(5)$ & $44(2)$ \\
\hline$C(14)$ & $4860(8)$ & $1401(5)$ & $-3308(5)$ & $40(2)$ \\
\hline$C(15)$ & $4003(7)$ & $1277(4)$ & $-2496(5)$ & 31 (1) \\
\hline$C(16)$ & $2777(6)$ & $671(3)$ & $-492(4)$ & $23(1)$ \\
\hline$C(17)$ & $1567(7)$ & $143(4)$ & $-857(5)$ & $28(1)$ \\
\hline$C(18)$ & $1718(7)$ & $-701(4)$ & $-709(5)$ & $34(1)$ \\
\hline$C(19)$ & $2998(7)$ & $-1004(4)$ & $-175(5)$ & $36(1)$ \\
\hline$C(20)$ & $4155(7)$ & $-497(4)$ & $206(5)$ & $36(1)$ \\
\hline$C(21)$ & $4080(6)$ & $341(3)$ & $41(4)$ & $26(1)$ \\
\hline$C(22)$ & $2778(6)$ & $1465(4)$ & $2055(4)$ & $24(1)$ \\
\hline$C(23)$ & $1931(8)$ & $732(4)$ & $2094(5)$ & $32(2)$ \\
\hline$C(24)$ & $2489(8)$ & $103(4)$ & $2721(5)$ & $38(2)$ \\
\hline$C(25)$ & $3869(8)$ & $212(5)$ & $3316(5)$ & $42(2)$ \\
\hline$C(26)$ & $4684(8)$ & $929(5)$ & $3285(5)$ & $40(2)$ \\
\hline$C(27)$ & $4182(7)$ & $1556(4)$ & $2653(5)$ & $32(1)$ \\
\hline$C(28)$ & $2317(7)$ & $3218(4)$ & $1909(5)$ & $25(1)$ \\
\hline$C(29)$ & $2024(8)$ & $3233(4)$ & $2880(5)$ & $38(1)$ \\
\hline$C(30)$ & $2050(10)$ & $3978(5)$ & $3395(6)$ & $52(2)$ \\
\hline$C(31)$ & $2360(10)$ & $4692(5)$ & $2909(6)$ & $50(2)$ \\
\hline$C(32)$ & $2644(8)$ & $4685(4)$ & $1929(6)$ & $40(2)$ \\
\hline$C(33)$ & $2629(7)$ & $3948(4)$ & $1412(5)$ & $30(1)$ \\
\hline $\mathrm{Cl}(2)$ & $1623(2)$ & $1643(2)$ & $-5087(1)$ & $57(1)$ \\
\hline $\mathrm{Cl}(3)$ & $-1583(2)$ & $1024(2)$ & $-5046(2)$ & $65(1)$ \\
\hline$C(34)$ & $100(10)$ & $1049(6)$ & $4312(6)$ & $55(2)$ \\
\hline$O(1)$ & $1320(10)$ & $-833(8)$ & $5260(10)$ & $131(4)$ \\
\hline$C(35)$ & $2190(10)$ & $-450(8)$ & $-3850(10)$ & $85(3)$ \\
\hline$C(36)$ & $6470(10)$ & $3960(8)$ & $3430(10)$ & $81(3)$ \\
\hline$C(37)$ & $6570(20)$ & $3300(10)$ & $4230(10)$ & $127(6)$ \\
\hline$C(38)$ & $1830(20)$ & $-1480(10)$ & $5160(10)$ & $115(5)$ \\
\hline
\end{tabular}

$U(e q)$ is defined as $1 / 3$ the trace of the Uij tensor. 
Table S6-3. Bond lengths (A) and angles (deg) for $6 \mathbf{e}$

$\begin{array}{llll}\text { Pt }(1)-\mathrm{N}(1) & 2.090(4) & \mathrm{Pt}(1)-\mathrm{P}(2) & 2.203(1) \\ \mathrm{Pt}(1)-\mathrm{C}(1) & 2.364(1) & \mathrm{Pt}(1)-\mathrm{Br}(1) & 2.4400(6) \\ \mathrm{P}(1)-\mathrm{N}(1) & 1.599(4) & \mathrm{P}(1)-\mathrm{C}(16) & 1.789(6) \\ \mathrm{P}(1)-\mathrm{C}(10) & 1.803(5) & \mathrm{P}(1)-\mathrm{C}(1) & 1.815(5) \\ \mathrm{P}(2)-\mathrm{C}(22) & 1.801(6) & \mathrm{P}(2)-\mathrm{C}(28) & 1.829(6) \\ \mathrm{P}(2)-\mathrm{C}(1) & 1.842(5) & \mathrm{N}(1)-\mathrm{C}(2) & 1.458(8) \\ \mathrm{C}(2)-\mathrm{C}(3) & 1.527(8) & \mathrm{C}(2)-\mathrm{C}(4) & 1.549(8) \\ \mathrm{C}(4)-\mathrm{C}(5) & 1.394(9) & \mathrm{C}(4)-\mathrm{C}(9) & 1.400(8) \\ \mathrm{C}(5)-\mathrm{C}(6) & 1.39(1) & \mathrm{C}(6)-\mathrm{C}(7) & 1.39(1) \\ \mathrm{C}(7)-\mathrm{C}(8) & 1.38(1) & \mathrm{C}(8)-\mathrm{C}(9) & 1.42(1) \\ \mathrm{C}(10)-\mathrm{C}(11) & 1.393(9) & \mathrm{C}(10)-\mathrm{C}(15) & 1.398(9) \\ \mathrm{C}(11)-\mathrm{C}(12) & 1.398(9) & \mathrm{C}(12)-\mathrm{C}(13) & 1.37(1) \\ \mathrm{C}(13)-\mathrm{C}(14) & 1.40(1) & \mathrm{C}(14)-\mathrm{C}(15) & 1.377(9) \\ \mathrm{C}(16)-\mathrm{C}(21) & 1.388(8) & \mathrm{C}(16)-\mathrm{C}(17) & 1.405(8) \\ \mathrm{C}(17)-\mathrm{C}(18) & 1.398(9) & \mathrm{C}(18)-\mathrm{C}(19) & 1.357(9) \\ \mathrm{C}(19)-\mathrm{C}(20) & 1.359(9) & \mathrm{C}(20)-\mathrm{C}(21) & 1.388(8) \\ \mathrm{C}(22)-\mathrm{C}(23) & 1.400(9) & \mathrm{C}(22)-\mathrm{C}(27) & 1.403(8) \\ \mathrm{C}(23)-\mathrm{C}(24) & 1.395(9) & \mathrm{C}(24)-\mathrm{C}(25) & 1.39(1) \\ \mathrm{C}(25)-\mathrm{C}(26) & 1.36(1) & \mathrm{C}(26)-\mathrm{C}(27) & 1.38(1) \\ \mathrm{C}(28)-\mathrm{C}(29) & 1.356(9) & \mathrm{C}(28)-\mathrm{C}(33) & 1.403(9) \\ \mathrm{C}(29)-\mathrm{C}(30) & 1.40(1) & \mathrm{C}(30)-\mathrm{C}(31) & 1.38(1) \\ \mathrm{C}(31)-\mathrm{C}(32) & 1.37(1) & \mathrm{C}(32)-\mathrm{C}(33) & 1.393(9) \\ \mathrm{C} 1(2)-\mathrm{C}(34) \# 1 & 1.766(9) & \mathrm{C} 1(3)-\mathrm{C}(34) \# 1 & 1.731(8) \\ \mathrm{C}(34)-\mathrm{Cl}(3) \# 1 & 1.731(8) & \mathrm{C}(34)-\mathrm{C}(12) \# 1 & 1.766(9) \\ \mathrm{O}(1)-\mathrm{C}(38) & 1.16(2) & \mathrm{O}(1)-\mathrm{C}(35) \# 1 & 1.51(2) \\ \mathrm{C}(35)-\mathrm{O}(1) \# 1 & 1.51(2) & \mathrm{C}(35)-\mathrm{C}(36) \# 2 & 1.57(2) \\ \mathrm{C}(36)-\mathrm{C}(37) & 1.53(2) & \mathrm{C}(36)-\mathrm{C}(35) \# 2 & 1.57(2) \\ \mathrm{C}(37)-\mathrm{C}(38) \# 2 & 1.58(2) & \mathrm{C}(38)-\mathrm{C}(37) \# 2 & 1.58(2) \\ & & & \end{array}$

$\mathrm{N}(1)-\mathrm{Pt}(1)-\mathrm{P}(2)$
$\mathrm{P}(2)-\mathrm{Pt}(1)-\mathrm{C}(1)$
$\mathrm{P}(2)-\mathrm{Pt}(1)-\mathrm{Br}(1)$
$\mathrm{N}(1)-\mathrm{P}(1)-\mathrm{C}(16)$
$\mathrm{C}(16)-\mathrm{P}(1)-\mathrm{C}(10)$
$\mathrm{C}(16)-\mathrm{P}(1)-\mathrm{C}(1)$
$\mathrm{C}(22)-\mathrm{P}(2)-\mathrm{C}(28)$
$\mathrm{C}(28)-\mathrm{P}(2)-\mathrm{C}(1)$
$\mathrm{C}(28)-\mathrm{P}(2)-\mathrm{Pt}(1)$
$\mathrm{C}(2)-\mathrm{N}(1)-\mathrm{P}(1)$
$\mathrm{P}(1)-\mathrm{N}(1)-\mathrm{Pt}(1)$
$\mathrm{N}(1)-\mathrm{C}(2)-\mathrm{C}(3)$
$\mathrm{C}(3)-\mathrm{C}(2)-\mathrm{C}(4)$
$\mathrm{C}(5)-\mathrm{C}(4)-\mathrm{C}(2)$
$\mathrm{C}(6)-\mathrm{C}(5)-\mathrm{C}(4)$
$\mathrm{C}(8)-\mathrm{C}(7)-\mathrm{C}(6)$
$\mathrm{C}(4)-\mathrm{C}(9)-\mathrm{C}(8)$
$\mathrm{C}(11)-\mathrm{C}(10)-\mathrm{P}(1)$
$\mathrm{C}(10)-\mathrm{C}(11)-\mathrm{C}(12)$
$\mathrm{C}(12)-\mathrm{C}(13)-\mathrm{C}(14)$
$\mathrm{C}(14)-\mathrm{C}(15)-\mathrm{C}(10)$
$\mathrm{C}(21)-\mathrm{C}(16)-\mathrm{P}(1)$
$\mathrm{C}(18)-\mathrm{C}(17)-\mathrm{C}(16)$
$\mathrm{C}(18)-\mathrm{C}(19)-\mathrm{C}(20)$
$\mathrm{C}(20)-\mathrm{C}(21)-\mathrm{C}(16)$
$\mathrm{C}(23)-\mathrm{C}(22)-\mathrm{P}(2)$
$\mathrm{C}(24)-\mathrm{C}(23)-\mathrm{C}(22)$
$\mathrm{C}(26)-\mathrm{C}(25)-\mathrm{C}(24)$
$\mathrm{C}(26)-\mathrm{C}(27)-\mathrm{C}(22)$
$\mathrm{C}(29)-\mathrm{C}(28)-\mathrm{P}(2)$
$\mathrm{C}(28)-\mathrm{C}(29)-\mathrm{C}(30)$
$\mathrm{C}(32)-\mathrm{C}(31)-\mathrm{C}(30)$
$\mathrm{C}(32)-\mathrm{C}(33)-\mathrm{C}(28)$
$\mathrm{C}(38)-\mathrm{O}(1)-\mathrm{C}(35) \# 1$
$\mathrm{C}(37)-\mathrm{C}(36)-\mathrm{C}(35) \# 2$
$\mathrm{O}(1)-\mathrm{C}(38)-\mathrm{C}(37) \# 2$

$89.9(1)$

$175.28(5)$

$91.29(4)$

$111.5(3)$

$107.0(3)$

107.4(3)

$105.8(3)$

$106.2(3)$

$117.9(2)$

$130.0(3)$

$110.6(2)$

$111.8(5)$

114.2 (5)

$117.4(5)$

$121.2(6)$

$120.8(7)$

$118.2(7)$

$120.3(5)$

$119.2(6)$

$120.7(6)$

$120.9(6)$

$121.9(4)$

$119.8(6)$

$120.7(6)$

$119.5(5)$

$119.7(4)$

$120.0(6)$

$120.2(6)$

$119.5(6)$

119.7 (5)

$120.0(7)$

$120.8(7)$

$119.3(6)$

$108(1)$

$100(1)$

$117(2)$
$\mathrm{N}(1)-\mathrm{Pt}(1)-\mathrm{Cl}(1)$

$\mathrm{N}(1)-\mathrm{Pt}(1)-\mathrm{Br}(1)$

$\mathrm{Cl}(1)-\mathrm{Pt}(1)-\mathrm{Br}(1)$

$\mathrm{N}(1)-\mathrm{P}(1)-\mathrm{C}(10)$

$\mathrm{N}(1)-\mathrm{P}(1)-\mathrm{C}(1)$

$\mathrm{C}(10)-\mathrm{P}(1)-\mathrm{C}(1)$

$\mathrm{C}(22)-\mathrm{P}(2)-\mathrm{C}(1)$

$\mathrm{C}(22)-\mathrm{P}(2)-\mathrm{Pt}(1)$

$\mathrm{C}(1)-\mathrm{P}(2)-\mathrm{Pt}(1)$

$\mathrm{C}(2)-\mathrm{N}(1)-\mathrm{Pt}(1)$

$P(1)-C(1)-P(2)$

$\mathrm{N}(1)-\mathrm{C}(2)-\mathrm{C}(4)$

$\mathrm{C}(5)-\mathrm{C}(4)-\mathrm{C}(9)$

$C(9)-C(4)-C(2)$

$C(5)-C(6)-C(7)$

$C(7)-C(8)-C(9)$

$C(11)-C(10)-C(15)$

$\mathrm{C}(15)-\mathrm{C}(10)-\mathrm{P}(1)$

$\mathrm{C}(13)-\mathrm{C}(12)-\mathrm{C}(11)$

$C(15)-C(14)-C(13)$

$\mathrm{C}(21)-\mathrm{C}(16)-\mathrm{C}(17)$

$\mathrm{C}(17)-\mathrm{C}(16)-\mathrm{P}(1)$

$\mathrm{C}(19)-\mathrm{C}(18)-\mathrm{C}(17)$

$C(19)-C(20)-C(21)$

$C(23)-C(22)-C(27)$

$\mathrm{C}(27)-\mathrm{C}(22)-\mathrm{P}(2)$

$C(25)-C(24)-C(23)$

$C(25)-C(26)-C(27)$

$\mathrm{C}(29)-\mathrm{C}(28)-\mathrm{C}(33)$

$\mathrm{C}(33)-\mathrm{C}(28)-\mathrm{P}(2)$

$C(31)-C(30)-C(29)$

C (31) -C (32)-C (33)

Cl (3) \#1-C (34)-Cl (2) \#1

$\mathrm{O}(1) \# 1-\mathrm{C}(35)-\mathrm{C}(36) \# 2$

C $(36)-C(37)-C(38)$ \# 2
$89.8(1)$

$175.8(2)$

$89.35(4)$

$114.3(2)$

$107.8(3)$

$108.6(3)$

$104.3(2)$

114.5 (2)

107.1 (2)

$118.3(3)$

$104.6(3)$

$111.8(5)$

$120.1(6)$

$122.5(5)$

$119.1(7)$

$120.6(7)$

$119.5(5)$

$120.2(5)$

$120.6(6)$

$119.1(7)$

$118.8(5)$

$119.2(4)$

$119.9(6)$

$121.1(6)$

$119.2(6)$

$121.1(5)$

$119.8(7)$

$121.4(6)$

$120.2(6)$

$119.7(5)$

$119.7(7)$

$120.0(7)$

112.7 (5)

$110(1)$

$102(1)$ 
Estimated standard deviations are given in the parenthesis.

Symmetry operators : : $1: x, y, z$

$2:-x, y+1 / 2,-z$ 
Table S6-4. Anisotropic displacement parameters (A^2 x 10^3) for

\begin{tabular}{|c|c|c|c|c|c|c|}
\hline atom & $\mathrm{U} 11$ & $\mathrm{U} 22$ & U33 & $\mathrm{U} 23$ & U13 & $\mathrm{U} 12$ \\
\hline Pt (1) & $18(1)$ & $17(1)$ & $27(1)$ & $1(1)$ & $4(1)$ & $0(1)$ \\
\hline Cl (1) & $15(1)$ & $27(1)$ & $35(1)$ & $0(1)$ & $3(1)$ & $-2(1)$ \\
\hline $\operatorname{Br}(1)$ & $32(1)$ & $39(1)$ & $35(1)$ & $4(1)$ & $14(1)$ & $5(1)$ \\
\hline$P(1)$ & $19(1)$ & $17(1)$ & $23(1)$ & $0(1)$ & $4(1)$ & $-1(1)$ \\
\hline$P(2)$ & 21 (1) & $17(1)$ & $25(1)$ & $-1(1)$ & $4(1)$ & $-1(1)$ \\
\hline $\mathrm{N}(1)$ & $18(2)$ & $13(2)$ & $36(2)$ & $2(3)$ & $5(1)$ & $-1(3)$ \\
\hline$C(1)$ & $21(2)$ & $25(2)$ & $25(3)$ & $1(2)$ & $1(2)$ & $-2(2)$ \\
\hline$C(2)$ & $22(2)$ & $29(3)$ & $25(3)$ & $2(2)$ & $2(2)$ & $4(2)$ \\
\hline$C(3)$ & $32(3)$ & $34(5)$ & $33(3)$ & $-3(2)$ & $-1(2)$ & $0(2)$ \\
\hline$C(4)$ & $23(2)$ & $24(3)$ & $33(3)$ & $5(2)$ & $-1(2)$ & $3(2)$ \\
\hline$C(5)$ & $31(3)$ & $28(3)$ & $30(3)$ & $0(2)$ & $2(2)$ & $6(2)$ \\
\hline$C(6)$ & $35(3)$ & 21 (3) & $67(5)$ & $-1(3)$ & $-3(3)$ & $2(2)$ \\
\hline$C(7)$ & $39(4)$ & $26(3)$ & $74(5)$ & $14(3)$ & $9(3)$ & $4(3)$ \\
\hline$C(8)$ & $51(4)$ & $46(5)$ & $73(6)$ & $42(4)$ & $23(4)$ & $9(3)$ \\
\hline$C(9)$ & $42(3)$ & $34(3)$ & $31(3)$ & $7(2)$ & $8(2)$ & $8(3)$ \\
\hline$C(10)$ & $14(2)$ & $27(4)$ & $24(2)$ & $2(2)$ & $2(2)$ & $3(2)$ \\
\hline C (11) & $33(3)$ & $28(3)$ & $36(3)$ & $4(3)$ & $7(2)$ & $-4(2)$ \\
\hline C (12) & $33(3)$ & $40(4)$ & $40(4)$ & $16(3)$ & $9(3)$ & $-6(3)$ \\
\hline C (13) & $33(3)$ & $67(7)$ & $34(3)$ & $16(3)$ & 15 (2) & $-1(3)$ \\
\hline$C(14)$ & $41(4)$ & $53(4)$ & $27(3)$ & $-3(3)$ & $12(3)$ & $6(3)$ \\
\hline$C(15)$ & $34(3)$ & $29(3)$ & 31 (3) & $-4(2)$ & $12(2)$ & $0(2)$ \\
\hline$C(16)$ & $24(2)$ & $19(2)$ & $26(3)$ & $2(2)$ & $9(2)$ & $-1(2)$ \\
\hline$C(17)$ & $33(3)$ & $22(3)$ & $29(3)$ & $-4(2)$ & $3(2)$ & $-4(2)$ \\
\hline C (18) & 31 (3) & $25(3)$ & $46(4)$ & $-3(3)$ & $8(3)$ & $-5(2)$ \\
\hline C (19) & 31 (3) & $21(3)$ & $59(4)$ & $3(3)$ & $16(3)$ & $3(2)$ \\
\hline$C(20)$ & $34(3)$ & $22(3)$ & $54(4)$ & $12(3)$ & $6(3)$ & $6(2)$ \\
\hline C (21) & $20(2)$ & $20(3)$ & $39(3)$ & $4(2)$ & $4(2)$ & $-4(2)$ \\
\hline C (22) & $25(2)$ & $21(2)$ & $26(3)$ & $-1(2)$ & $4(2)$ & $3(2)$ \\
\hline C (23) & $33(3)$ & $24(3)$ & $38(4)$ & $3(3)$ & $2(3)$ & $4(3)$ \\
\hline C (24) & $44(4)$ & $27(3)$ & $44(4)$ & $13(3)$ & $7(3)$ & $6(3)$ \\
\hline$C(25)$ & $49(4)$ & $39(4)$ & $39(4)$ & $13(3)$ & $7(3)$ & $14(3)$ \\
\hline C (26) & $37(3)$ & $43(4)$ & $38(4)$ & $-1(3)$ & $-4(3)$ & $6(3)$ \\
\hline C (27) & $27(3)$ & $33(3)$ & $35(3)$ & $-2(2)$ & $-1(2)$ & $1(2)$ \\
\hline C (28) & $20(3)$ & $23(3)$ & $32(3)$ & $-5(2)$ & $-2(2)$ & $-4(2)$ \\
\hline C (29) & $56(4)$ & $29(3)$ & 32 (3) & $-7(3)$ & 12 (3) & $-1(3)$ \\
\hline$C(30)$ & $78(5)$ & $41(4)$ & $39(4)$ & $-11(3)$ & $17(4)$ & $2(4)$ \\
\hline C (31) & $64(5)$ & $27(3)$ & $60(5)$ & $-15(3)$ & $4(4)$ & $1(3)$ \\
\hline C (32) & $46(4)$ & $22(3)$ & $50(4)$ & $0(3)$ & $-2(3)$ & $1(3)$ \\
\hline C (33) & $32(3)$ & $23(3)$ & $34(3)$ & $0(2)$ & $-2(2)$ & $-3(2)$ \\
\hline $\mathrm{Cl}(2)$ & $50(1)$ & $77(1)$ & $44(1)$ & $4(1)$ & $11(1)$ & $-5(1)$ \\
\hline $\mathrm{Cl}(3)$ & $50(1)$ & $102(2)$ & $43(1)$ & $-21(1)$ & 7 (1) & $-14(1)$ \\
\hline C (34) & $57(5)$ & 71 ( 6) & $38(4)$ & $-6(4)$ & $12(3)$ & $5(4)$ \\
\hline
\end{tabular}

The anisotropic displacement factor exponent takes the form $2 \mathrm{pi}^{\wedge} 2\left[\mathrm{~h}^{\wedge} 2 \mathrm{a} * \wedge 2 \mathrm{U}(11)+\ldots+2 \mathrm{hka} * \mathrm{~b} * \mathrm{U}(12)\right]$ 
Table S6-5. Hydrogen Coordinates (A x 10^4) and equivalent isotropic displacement parameters $\left(A^{\wedge} 2 \times 10^{\wedge} 3\right)$ for $6 e$

\begin{tabular}{|c|c|c|c|c|}
\hline atom & $\mathrm{x}$ & $\mathrm{y}$ & $\mathrm{z}$ & $\mathrm{U}(\mathrm{eq})$ \\
\hline $\mathrm{H}(1 \mathrm{~A})$ & 3794 & 2861 & 95 & 29 \\
\hline $\mathrm{H}(1 \mathrm{~B})$ & 4548 & 2020 & 528 & 29 \\
\hline $\mathrm{H}(2)$ & -1072 & 2559 & -1561 & 30 \\
\hline $\mathrm{H}(3 \mathrm{~A})$ & 778 & 1778 & -2952 & 49 \\
\hline$H(3 B)$ & -995 & 2099 & -3149 & 49 \\
\hline $\mathrm{H}(3 \mathrm{C})$ & -625 & 1329 & -2439 & 49 \\
\hline $\mathrm{H}(5)$ & 625 & 3698 & -606 & 36 \\
\hline $\mathrm{H}(6)$ & 1729 & 4981 & -864 & 50 \\
\hline $\mathrm{H}(7)$ & 2542 & 5314 & -2430 & 55 \\
\hline $\mathrm{H}(8)$ & 2203 & 4394 & -3730 & 67 \\
\hline $\mathrm{H}(9)$ & 1085 & 3082 & -3481 & 42 \\
\hline $\mathrm{H}(11)$ & 4293 & 3114 & -1535 & 39 \\
\hline $\mathrm{H}(12)$ & 5804 & 3310 & -2902 & 45 \\
\hline $\mathrm{H}(13)$ & 6107 & 2265 & -4016 & 53 \\
\hline $\mathrm{H}(14)$ & 5001 & 969 & -3763 & 48 \\
\hline $\mathrm{H}(15)$ & 3561 & 754 & -2388 & 37 \\
\hline $\mathrm{H}(17)$ & 648 & 359 & -1203 & 33 \\
\hline $\mathrm{H}(18)$ & 926 & -1062 & -983 & 41 \\
\hline $\mathrm{H}(19)$ & 3087 & -1577 & -66 & 44 \\
\hline $\mathrm{H}(20)$ & 5029 & -719.9999 & 592 & 44 \\
\hline $\mathrm{H}(21)$ & 4915 & 686 & 291 & 32 \\
\hline $\mathrm{H}(23)$ & 977 & 663 & 1694 & 38 \\
\hline $\mathrm{H}(24)$ & 1925 & -398 & 2741 & 46 \\
\hline $\mathrm{H}(25)$ & 4248 & -215 & 3747 & 51 \\
\hline $\mathrm{H}(26)$ & 5616 & 1000 & 3705 & 48 \\
\hline $\mathrm{H}(27)$ & 4784 & 2045 & 2624 & 38 \\
\hline $\mathrm{H}(29)$ & 1799 & 2739 & 3211 & 46 \\
\hline $\mathrm{H}(30)$ & 1858 & 3989 & 4077 & 62 \\
\hline $\mathrm{H}(31)$ & 2382 & 5197 & 3259 & 60 \\
\hline $\mathrm{H}(32)$ & 2850 & 5183 & 1600 & 48 \\
\hline $\mathrm{H}(33)$ & 2827 & 3939 & 731 & 36 \\
\hline $\mathrm{H}(34 \mathrm{~A})$ & -158 & 1277 & 3644 & 66 \\
\hline $\mathrm{H}(34 \mathrm{~B})$ & 491 & 483 & 4232 & 66 \\
\hline $\mathrm{H}(35 \mathrm{~A})$ & 1441 & -344 & -3331 & 103 \\
\hline $\mathrm{H}(35 \mathrm{~B})$ & 2649 & 79 & -4032 & 103 \\
\hline $\mathrm{H}(36 \mathrm{~A})$ & 5431 & 4234 & 3375 & 97 \\
\hline $\mathrm{H}(36 \mathrm{~B})$ & 6697 & 3735 & 2772 & 97 \\
\hline $\mathrm{H}(37 \mathrm{~A})$ & 6603 & 2744 & 3940 & 152 \\
\hline $\mathrm{H}(37 \mathrm{~B})$ & 5662 & 3334 & 4650 & 152 \\
\hline $\mathrm{H}(38 \mathrm{~A})$ & 1987 & -1561 & 4449 & 138 \\
\hline $\mathrm{H}(38 \mathrm{~B})$ & 1025 & -1885 & 5338 & 138 \\
\hline
\end{tabular}


Figure S6: Molecular structure of 7a

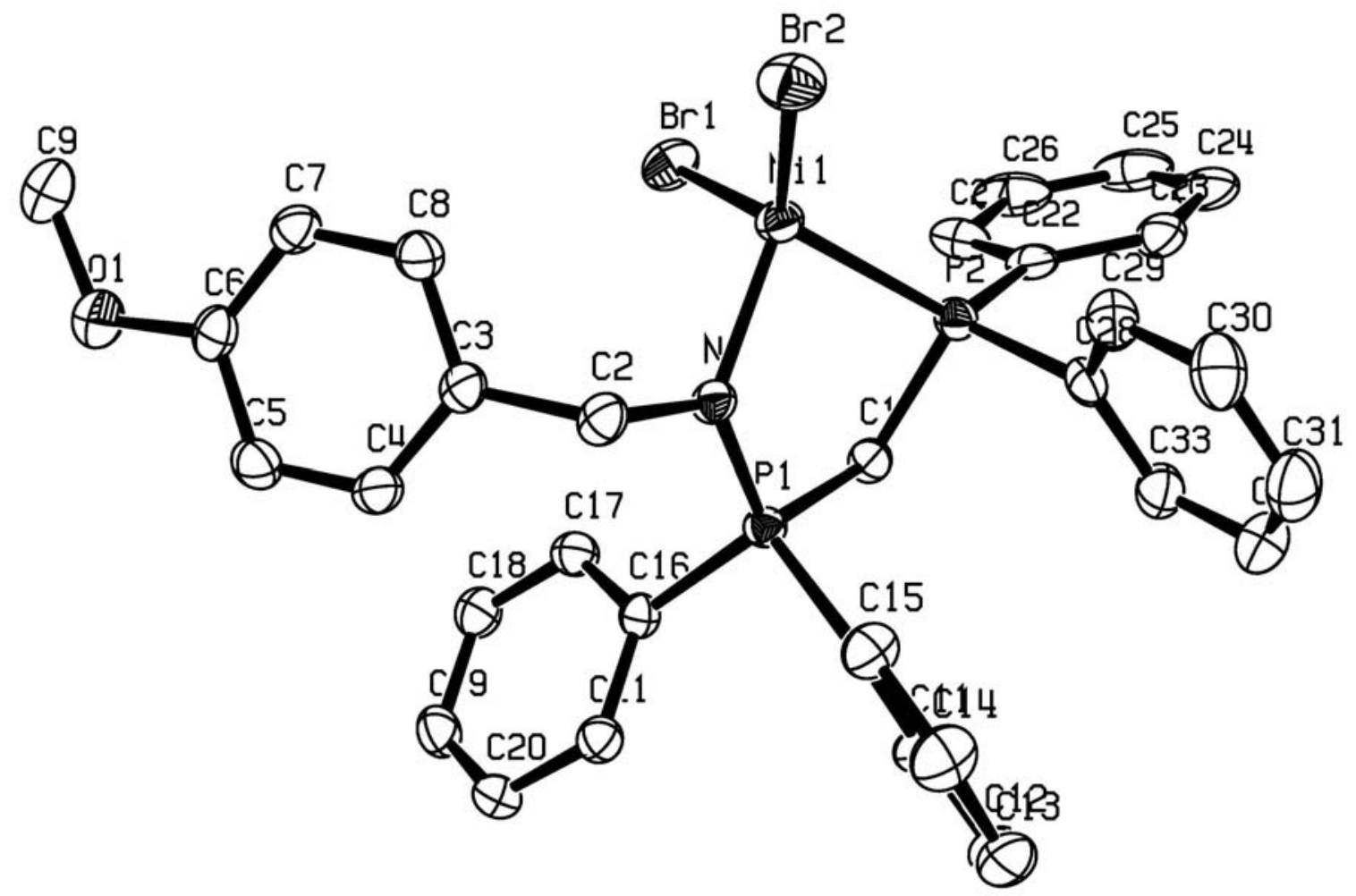


Table S7-1. Crystal data for 7a

\begin{tabular}{|c|c|}
\hline Compound & $7 \mathbf{a}$ \\
\hline Molecular formula & $\mathrm{C}_{33} \mathrm{H}_{31} \mathrm{Br}_{2} \mathrm{NNiOP}_{2}, \mathrm{C}_{4} \mathrm{H}_{8} \mathrm{O}$ \\
\hline Molecular weight & 810.16 \\
\hline Crystal habit & blue plate \\
\hline Crystal dimensions $(\mathrm{mm})$ & $0.22 \times 0.18 \times 0.03$ \\
\hline Crystal system & monoclinic \\
\hline Space group & $\mathrm{P} 2_{1} / \mathrm{c}$ \\
\hline $\mathrm{a}(\AA)$ & $19.9970(10)$ \\
\hline $\mathrm{b}(\AA)$ & $9.7900(10)$ \\
\hline$c(\AA)$ & $17.6700(10)$ \\
\hline$\alpha\left(^{\circ}\right)$ & 90.00 \\
\hline$\beta\left(^{\circ}\right)$ & $95.4000(10)$ \\
\hline$\gamma\left({ }^{\circ}\right)$ & 90.00 \\
\hline $\mathrm{V}\left(\AA^{3}\right)$ & $3443.9(4)$ \\
\hline $\mathrm{Z}$ & 4 \\
\hline$d\left(g-\mathrm{cm}^{-3}\right)$ & 1.563 \\
\hline $\mathrm{F}(000)$ & 1648 \\
\hline$\mu\left(\mathrm{cm}^{-1}\right)$ & 3.011 \\
\hline Absorption corrections & multi-scan ; $0.5571 \mathrm{~min}, 0.9151 \mathrm{max}$ \\
\hline Diffractometer & KappaCCD \\
\hline X-ray source & MoK $\alpha$ \\
\hline$\lambda(\AA)$ & 0.71069 \\
\hline Monochromator & graphite \\
\hline $\mathrm{T}(\mathrm{K})$ & $150.0(1)$ \\
\hline Scan mode & phi and omega scans \\
\hline Maximum $\theta$ & 27.47 \\
\hline HKL ranges & $-2525 ;-1012 ;-2222$ \\
\hline Reflections measured & 14102 \\
\hline Unique data & 7871 \\
\hline Rint & 0.0264 \\
\hline Reflections used & 5916 \\
\hline Criterion & $\mathrm{I}>2 \sigma \mathrm{I})$ \\
\hline Refinement type & Fsqd \\
\hline Hydrogen atoms & mixed \\
\hline Parameters refined & 407 \\
\hline Reflections / parameter & 14 \\
\hline wR2 & 0.0903 \\
\hline $\mathrm{R} 1$ & 0.0350 \\
\hline Weights $a, b$ & $0.0425 ; 0.1060$ \\
\hline GoF & 1.039 \\
\hline difference peak / hole $\left(\mathrm{e} \AA^{-3}\right)$ & $0.907(0.079) /-0.669(0.079)$ \\
\hline
\end{tabular}


Table S7-2. Atomic Coordinates (A x 10^4) and equivalent isotropic displacement parameters $\left(A^{\wedge} 2 \times 10^{\wedge} 3\right)$ for $7 a$

\begin{tabular}{|c|c|c|c|c|}
\hline atom & $\mathrm{x}$ & Y & z & $\mathrm{U}(\mathrm{eq})$ \\
\hline $\operatorname{Br}(1)$ & $1558(1)$ & $955(1)$ & $3056(1)$ & $32(1)$ \\
\hline $\operatorname{Br}(2)$ & $3529(1)$ & $458(1)$ & $4046(1)$ & $36(1)$ \\
\hline $\mathrm{Ni}(1)$ & $2597(1)$ & $-229(1)$ & $3221(1)$ & $23(1)$ \\
\hline $\mathrm{P}(1)$ & $2090(1)$ & $-3177(1)$ & $2796(1)$ & $19(1)$ \\
\hline$P(2)$ & $2662(1)$ & $-868(1)$ & $1977(1)$ & $21(1)$ \\
\hline$O(1)$ & $182(1)$ & $-1195(2)$ & $5835(1)$ & $37(1)$ \\
\hline $\mathrm{N}(1)$ & $2380(1)$ & $-2157(2)$ & $3451(1)$ & $21(1)$ \\
\hline$C(1)$ & $1994(1)$ & $-2162(2)$ & $1937(1)$ & $21(1)$ \\
\hline$C(2)$ & $2433(2)$ & $-2624(3)$ & $4257(1)$ & $26(1)$ \\
\hline$C(3)$ & $1840(1)$ & $-2208(2)$ & $4685(1)$ & $23(1)$ \\
\hline$C(4)$ & $1339(2)$ & $-3137(3)$ & $4814(1)$ & $28(1)$ \\
\hline$C(5)$ & $797(2)$ & $-2780(3)$ & $5197(1)$ & $29(1)$ \\
\hline$C(6)$ & $743(2)$ & $-1455(3)$ & $5473(1)$ & $26(1)$ \\
\hline$C(7)$ & $1237(2)$ & $-508(3)$ & $5353(2)$ & $26(1)$ \\
\hline$C(8)$ & $1782(2)$ & $-888(3)$ & $4962(1)$ & $26(1)$ \\
\hline$C(9)$ & $126(2)$ & $112(3)$ & $6174(2)$ & $37(1)$ \\
\hline$C(10)$ & $2625(1)$ & $-4605(2)$ & $2626(1)$ & $20(1)$ \\
\hline$C(11)$ & $2459(2)$ & $-5444(3)$ & $1996(2)$ & $28(1)$ \\
\hline$C(12)$ & $2863(2)$ & $-6550(3)$ & $1860(2)$ & $33(1)$ \\
\hline$C(13)$ & $3412(2)$ & $-6864(3)$ & $2361(2)$ & $36(1)$ \\
\hline$C(14)$ & $3578(2)$ & $-6034(3)$ & $2983(2)$ & $37(1)$ \\
\hline$C(15)$ & $3189(2)$ & $-4894(3)$ & $3105(2)$ & $30(1)$ \\
\hline$C(16)$ & $1283(1)$ & $-3918(2)$ & $2937(1)$ & $21(1)$ \\
\hline$C(17)$ & $724(1)$ & $-3057(3)$ & $2927(1)$ & $25(1)$ \\
\hline$C(18)$ & $110(2)$ & $-3568(3)$ & $3090(2)$ & $29(1)$ \\
\hline$C(19)$ & $56(2)$ & $-4937(3)$ & $3292(2)$ & $29(1)$ \\
\hline$C(20)$ & $604(1)$ & $-5790(3)$ & $3318(2)$ & $27(1)$ \\
\hline$C(21)$ & $1217(1)$ & $-5285(2)$ & $3135(1)$ & $24(1)$ \\
\hline C (22) & $2427(2)$ & $180(2)$ & $1139(1)$ & $26(1)$ \\
\hline$C(23)$ & $2892(2)$ & $471(3)$ & $616(2)$ & $34(1)$ \\
\hline$C(24)$ & $2704(2)$ & $1336(3)$ & $7(2)$ & $46(1)$ \\
\hline C (25) & $2070(2)$ & $1898(3)$ & $-87(2)$ & $51(1)$ \\
\hline$C(26)$ & $1612(2)$ & $1621(3)$ & $429(2)$ & $44(1)$ \\
\hline$C(27)$ & $1794(2)$ & $766(3)$ & $1046(2)$ & $31(1)$ \\
\hline$C(28)$ & $3413(1)$ & $-1795(2)$ & $1791(1)$ & $23(1)$ \\
\hline$C(29)$ & $4004(2)$ & $-1541(3)$ & $2249(2)$ & $32(1)$ \\
\hline$C(30)$ & $4584(2)$ & $-2266(3)$ & $2131(2)$ & $41(1)$ \\
\hline$C(31)$ & $4574(2)$ & $-3234(3)$ & $1564(2)$ & $41(1)$ \\
\hline$C(32)$ & $3990(2)$ & $-3488(3)$ & $1108(2)$ & $39(1)$ \\
\hline C (33) & $3409(2)$ & $-2775(3)$ & $1220(2)$ & $30(1)$ \\
\hline$O(2)$ & $4416(2)$ & $-8481(3)$ & $1222(2)$ & $81(1)$ \\
\hline$C(34)$ & $4388(3)$ & $-7234(5)$ & $807(3)$ & $114(2)$ \\
\hline$C(35)$ & $4904(2)$ & $-7153(5)$ & $357(3)$ & $89(2)$ \\
\hline$C(36)$ & $5443(2)$ & $-8119(4)$ & $675(2)$ & $61(1)$ \\
\hline$C(37)$ & $5038(2)$ & $-9126(3)$ & $1102(2)$ & $60(1)$ \\
\hline
\end{tabular}

$\mathrm{U}(\mathrm{eq})$ is defined as $1 / 3$ the trace of the Uij tensor. 
Table S7-3. Bond lengths (A) and angles (deg) for 7a

$$
\begin{aligned}
& \mathrm{Br}(1)-\mathrm{Ni}(1) \\
& \mathrm{Ni}(1)-\mathrm{N}(1) \\
& \mathrm{P}(1)-\mathrm{N}(1) \\
& \mathrm{P}(1)-\mathrm{C}(16) \\
& \mathrm{P}(2)-\mathrm{C}(28) \\
& \mathrm{P}(2)-\mathrm{C}(1) \\
& \mathrm{O}(1)-\mathrm{C}(9) \\
& \mathrm{C}(2)-\mathrm{C}(3) \\
& \mathrm{C}(3)-\mathrm{C}(8) \\
& \mathrm{C}(5)-\mathrm{C}(6) \\
& \mathrm{C}(7)-\mathrm{C}(8) \\
& \mathrm{C}(10)-\mathrm{C}(11) \\
& \mathrm{C}(12)-\mathrm{C}(13) \\
& \mathrm{C}(14)-\mathrm{C}(15) \\
& \mathrm{C}(16)-\mathrm{C}(17) \\
& \mathrm{C}(18)-\mathrm{C}(19) \\
& \mathrm{C}(20)-\mathrm{C}(21) \\
& \mathrm{C}(22)-\mathrm{C}(23) \\
& \mathrm{C}(24)-\mathrm{C}(25) \\
& \mathrm{C}(26)-\mathrm{C}(27) \\
& \mathrm{C}(28)-\mathrm{C}(29) \\
& \mathrm{C}(30)-\mathrm{C}(31) \\
& \mathrm{C}(32)-\mathrm{C}(33) \\
& \mathrm{O}(2)-\mathrm{C}(37) \\
& \mathrm{C}(35)-\mathrm{C}(36) \\
&
\end{aligned}
$$

$\mathrm{N}(1)-\mathrm{Ni}(1)-\mathrm{P}(2)$

$\mathrm{P}(2)-\mathrm{Ni}(1)-\mathrm{Br}(2)$

$\mathrm{P}(2)-\mathrm{Ni}(1)-\mathrm{Br}(1)$

$\mathrm{N}(1)-\mathrm{P}(1)-\mathrm{C}(10)$

$\mathrm{C}(10)-\mathrm{P}(1)-\mathrm{C}(16)$

$\mathrm{C}(10)-\mathrm{P}(1)-\mathrm{C}(1)$

$\mathrm{C}(28)-\mathrm{P}(2)-\mathrm{C}(22)$

$\mathrm{C}(22)-\mathrm{P}(2)-\mathrm{C}(1)$

$\mathrm{C}(22)-\mathrm{P}(2)-\mathrm{Ni}(1)$

$\mathrm{C}(6)-\mathrm{O}(1)-\mathrm{C}(9)$

$\mathrm{C}(2)-\mathrm{N}(1)-\mathrm{Ni}(1)$

$\mathrm{P}(1)-\mathrm{C}(1)-\mathrm{P}(2)$

$C(4)-C(3)-C(8)$

$C(8)-C(3)-C(2)$

$C(4)-C(5)-C(6)$

$O(1)-C(6)-C(5)$

$C(6)-C(7)-C(8)$

$C(15)-C(10)-C(11)$

$\mathrm{C}(11)-\mathrm{C}(10)-\mathrm{P}(1)$

$\mathrm{C}(13)-\mathrm{C}(12)-\mathrm{C}(11)$

$C(13)-C(14)-C(15)$

$C(21)-C(16)-C(17)$

$\mathrm{C}(17)-\mathrm{C}(16)-\mathrm{P}(1)$

C (17) -C (18)-C (19)

$C(19)-C(20)-C(21)$

$C(27)-C(22)-C(23)$

$\mathrm{C}(23)-\mathrm{C}(22)-\mathrm{P}(2)$

C (25) $-C(24)-C(23)$

$\mathrm{C}(25)-\mathrm{C}(26)-\mathrm{C}(27)$

C (33) $-\mathrm{C}(28)-\mathrm{C}(29)$

$\mathrm{C}(29)-\mathrm{C}(28)-\mathrm{P}(2)$

$\mathrm{C}(31)-\mathrm{C}(30)-\mathrm{C}(29)$

$\mathrm{C}(31)-\mathrm{C}(32)-\mathrm{C}(33)$

$\mathrm{C}(34)-\mathrm{O}(2)-\mathrm{C}(37)$

$C(34)-C(35)-C(36)$

$\mathrm{O}(2)-\mathrm{C}(37)-\mathrm{C}(36)$
$2.3743(5$

$1.987(2)$

$1.596(2)$

$1.807(3)$

$1.810(3)$

$1.838(3)$

$1.421(3)$

$1.521(4)$

$1.391(4)$

$1.393(4)$

$1.396(4)$

$1.398(4)$

$1.379(4)$

$1.389(4)$

$1.399(4)$

$1.394(4)$

$1.387(4)$

$1.401(4)$

$1.379(5)$

$1.395(4)$

1.392(4)

$1.378(4)$

$1.384(4)$

$1.429(5)$

$1.502(5)$

$88.35(6)$

$123.83(2)$

$98.21(2)$

$115.2(1)$

$105.3(1)$

$107.7(1)$

$106.8(1)$

$103.0(1)$

$126.05(8)$

$117.8(2)$

$119.3(2)$

$109.2(1)$

$117.8(3)$

$121.2(2)$

$120.0(3)$

$115.6(2)$

$119.9(2)$

$119.3(2)$

$119.2(2)$

$120.3(3)$

$120.0(3)$

$119.2(2)$

$118.6(2)$

$119.6(3)$

$119.6(2)$

119.5 (3)

$120.8(2)$

$120.9(3)$

$119.6(3)$

$119.4(3)$

$118.6(2)$

$120.3(3)$

$120.2(3)$

$107.2(3)$

$107.5(4)$

$107.4(3)$
$\mathrm{Br}(2)-\mathrm{Ni}(1)$

$\mathrm{Ni}(1)-\mathrm{P}(2)$

$P(1)-C(10)$

$\mathrm{P}(1)-\mathrm{C}(1)$

$P(2)-C(22)$

$\mathrm{O}(1)-\mathrm{C}(6)$

$\mathrm{N}(1)-\mathrm{C}(2)$

$C(3)-C(4)$

$C(4)-C(5)$

$C(6)-C(7)$

$C(10)-C(15)$

C (11) -C (12)

$C(13)-C(14)$

C (16) $-\mathrm{C}(21)$

$C(17)-C(18)$

$C(19)-C(20)$

C (22) $-\mathrm{C}(27)$

C (23) $-\mathrm{C}(24)$

C (25) $-C(26)$

$C(28)-C(33)$

$C(29)-C(30)$

C (31) $-\mathrm{C}(32)$

$\mathrm{O}(2)-\mathrm{C}(34)$

C (34) $-\mathrm{C}(35)$

C (36) $-\mathrm{C}(37)$

2.3545 ( 4 )

2. $3010(7)$

$1.802(2)$

$1.809(2)$

$1.825(3)$

$1.367(3)$

$1.488(3)$

$1.388(4)$

$1.376(4)$

$1.385(4)$

$1.374(4)$

$1.386(4)$

$1.382(4)$

$1.392(3)$

$1.382(4)$

$1.375(4)$

$1.385(4)$

$1.392(4)$

$1.378(5)$

$1.391(4)$

$1.392(4)$

$1.379(4)$

$1.423(5)$

$1.364(6)$

$1.519(5)$

$\mathrm{N}(1)-\mathrm{Ni}(1)-\mathrm{Br}(2)$

$108.68(6)$

$106.44(7)$

$124.71(2)$

$114.8(1)$

$105.5(1)$

$108.1(1)$

$105.0(1)$

$115.6(1)$

$96.70(8)$

$119.3(2)$

$121.1(1)$

$113.8(2)$

$121.0(2)$

$121.9(2)$

$125.1(2)$

$119.4(3)$

$121.2(3)$

$121.5(2)$

$119.9(3)$

$119.8(3)$

$120.6(3)$

$121.9(2)$

$120.3(2)$

$120.8(3)$

$120.6(3)$

$119.5(2)$

119.1 (3)

$120.2(3)$

$120.6(3)$

$122.0(2)$

$119.8(3)$

$120.1(3)$

$120.3(3)$

$110.8(4)$

$101.4(3)$ 
Table S7-4. Anisotropic displacement parameters ( $A^{\wedge} 2 \times 10^{\wedge} 3$ ) for

\begin{tabular}{|c|c|c|c|c|c|c|}
\hline atom & $\mathrm{U} 11$ & $\mathrm{U} 22$ & U33 & $\mathrm{U} 23$ & U13 & $\mathrm{U} 12$ \\
\hline $\operatorname{Br}(1)$ & $32(1)$ & $29(1)$ & $35(1)$ & $-4(1)$ & $1(1)$ & $8(1)$ \\
\hline $\operatorname{Br}(2)$ & $35(1)$ & $38(1)$ & $33(1)$ & $-5(1)$ & $-7(1)$ & $-7(1)$ \\
\hline $\mathrm{Ni}(1)$ & $27(1)$ & $20(1)$ & 21 (1) & $-2(1)$ & $1(1)$ & $-1(1)$ \\
\hline $\mathrm{P}(1)$ & 21 (1) & $17(1)$ & $18(1)$ & $1(1)$ & $1(1)$ & $0(1)$ \\
\hline$P(2)$ & $24(1)$ & $20(1)$ & $18(1)$ & $1(1)$ & $2(1)$ & $-2(1)$ \\
\hline$O(1)$ & $37(1)$ & $38(1)$ & $37(1)$ & $-4(1)$ & $12(1)$ & $0(1)$ \\
\hline $\mathrm{N}(1)$ & $27(1)$ & $19(1)$ & $16(1)$ & $0(1)$ & $2(1)$ & $1(1)$ \\
\hline$C(1)$ & $25(2)$ & $20(1)$ & $19(1)$ & $2(1)$ & $2(1)$ & $2(1)$ \\
\hline$C(2)$ & $33(2)$ & $26(1)$ & $19(1)$ & $1(1)$ & $2(1)$ & $5(1)$ \\
\hline$C(3)$ & $30(2)$ & $24(1)$ & $16(1)$ & $2(1)$ & $1(1)$ & $3(1)$ \\
\hline$C(4)$ & $43(2)$ & 21 (1) & $19(1)$ & $0(1)$ & $3(1)$ & $0(1)$ \\
\hline$C(5)$ & $38(2)$ & $27(1)$ & $23(1)$ & $3(1)$ & $3(1)$ & $-5(1)$ \\
\hline$C(6)$ & $30(2)$ & $32(2)$ & $17(1)$ & $1(1)$ & $2(1)$ & $4(1)$ \\
\hline$C(7)$ & $32(2)$ & $22(1)$ & $23(1)$ & $0(1)$ & $2(1)$ & $4(1)$ \\
\hline$C(8)$ & $32(2)$ & $25(1)$ & $22(1)$ & $1(1)$ & $3(1)$ & $-3(1)$ \\
\hline C (9) & $39(2)$ & $43(2)$ & $30(2)$ & $-5(1)$ & $7(1)$ & $9(1)$ \\
\hline$C(10)$ & $20(2)$ & $19(1)$ & $23(1)$ & $1(1)$ & $6(1)$ & $-1(1)$ \\
\hline C (11) & $28(2)$ & $24(1)$ & $31(2)$ & $-5(1)$ & $0(1)$ & $0(1)$ \\
\hline C (12) & $37(2)$ & $25(1)$ & $39(2)$ & $-11(1)$ & $10(1)$ & $-3(1)$ \\
\hline$C(13)$ & $33(2)$ & $26(2)$ & $49(2)$ & $-2(1)$ & $11(2)$ & $7(1)$ \\
\hline $\mathrm{C}(14)$ & $27(2)$ & $39(2)$ & $44(2)$ & $-2(1)$ & $-1(1)$ & $9(1)$ \\
\hline$C(15)$ & $27(2)$ & 31 (2) & 31 (2) & $-4(1)$ & $1(1)$ & $2(1)$ \\
\hline$C(16)$ & $22(2)$ & $23(1)$ & $18(1)$ & $1(1)$ & $3(1)$ & $0(1)$ \\
\hline C (17) & $28(2)$ & $23(1)$ & $25(1)$ & $0(1)$ & $2(1)$ & $0(1)$ \\
\hline C (18) & $25(2)$ & $30(2)$ & $32(2)$ & $-1(1)$ & $6(1)$ & $3(1)$ \\
\hline C (19) & $25(2)$ & $34(2)$ & 31 (2) & $0(1)$ & $7(1)$ & $-6(1)$ \\
\hline C (20) & $30(2)$ & $23(1)$ & $30(2)$ & $0(1)$ & $6(1)$ & $-6(1)$ \\
\hline C (21) & $25(2)$ & $25(1)$ & $23(1)$ & $-1(1)$ & $3(1)$ & $0(1)$ \\
\hline C (22) & $41(2)$ & $15(1)$ & $19(1)$ & $-2(1)$ & $0(1)$ & $-4(1)$ \\
\hline C (23) & $57(2)$ & $23(1)$ & $25(2)$ & $-2(1)$ & $13(2)$ & $-3(1)$ \\
\hline C (24) & $93(3)$ & $23(2)$ & $25(2)$ & $-1(1)$ & $15(2)$ & $-10(2)$ \\
\hline C (25) & $103(3)$ & $21(2)$ & $25(2)$ & $3(1)$ & $-13(2)$ & $-5(2)$ \\
\hline C (26) & $64(2)$ & $22(1)$ & $41(2)$ & $2(1)$ & $-21(2)$ & $2(2)$ \\
\hline C (27) & $39(2)$ & $23(1)$ & $30(2)$ & $2(1)$ & $-7(1)$ & $-7(1)$ \\
\hline C (28) & $24(2)$ & 25 (1) & 21 (1) & $4(1)$ & $6(1)$ & $-4(1)$ \\
\hline C (29) & $29(2)$ & $39(2)$ & $28(2)$ & $-1(1)$ & $4(1)$ & $-2(1)$ \\
\hline$C(30)$ & $24(2)$ & $58(2)$ & $40(2)$ & $2(2)$ & $5(1)$ & $2(2)$ \\
\hline C (31) & $34(2)$ & $45(2)$ & $44(2)$ & $4(1)$ & $13(2)$ & $9(2)$ \\
\hline C (32) & $40(2)$ & $38(2)$ & $40(2)$ & $-5(1)$ & $14(2)$ & $5(2)$ \\
\hline C (33) & $30(2)$ & $33(2)$ & $27(2)$ & $-3(1)$ & $6(1)$ & $-2(1)$ \\
\hline$O(2)$ & $56(2)$ & $71(2)$ & $118(3)$ & $47(2)$ & $17(2)$ & $6(2)$ \\
\hline C (34) & $74(4)$ & $118(4)$ & $158(5)$ & $102(4)$ & $48(4)$ & $38(3)$ \\
\hline C (35) & $55(3)$ & $98(3)$ & $118(4)$ & $67(3)$ & $26(3)$ & 25 (3) \\
\hline$C(36)$ & $47(3)$ & $70(3)$ & $66(3)$ & $7(2)$ & $5(2)$ & $15(2)$ \\
\hline C (37) & $67(3)$ & $35(2)$ & $76(3)$ & $4(2)$ & $-2(2)$ & $9(2)$ \\
\hline
\end{tabular}

The anisotropic displacement factor exponent takes the form $2 \mathrm{pi} 2\left[\mathrm{~h} \wedge 2 \mathrm{a} \star \wedge 2 \mathrm{U}(11)+\ldots+2 \mathrm{hka} \mathrm{b}^{\star} \mathrm{U}(12)\right]$ 
Table S7-5. Hydrogen Coordinates (A x 10^4) and equivalent isotropic displacement parameters $\left(A^{\wedge} 2 \times 10^{\wedge} 3\right)$ for $7 a$

\begin{tabular}{|c|c|c|c|c|}
\hline atom & $\mathrm{x}$ & $\mathrm{Y}$ & z & $\mathrm{U}(\mathrm{eq})$ \\
\hline $\mathrm{H}(1 \mathrm{~A})$ & 1549 & -1710 & 1890 & 26 \\
\hline $\mathrm{H}(1 \mathrm{~B})$ & 2022 & -2757 & 1488 & 26 \\
\hline $\mathrm{H}(2 \mathrm{~A})$ & 2849 & -2244 & 4524 & 31 \\
\hline $\mathrm{H}(2 \mathrm{~B})$ & 2473 & -3632 & 4267 & 31 \\
\hline $\mathrm{H}(4)$ & 1371 & -4046 & 4632 & 33 \\
\hline $\mathrm{H}(5)$ & 460 & -3436 & 5273 & 35 \\
\hline $\mathrm{H}(7)$ & 1204 & 399 & 5536 & 31 \\
\hline $\mathrm{H}(8)$ & 2120 & -234 & 4884 & 32 \\
\hline $\mathrm{H}(9 \mathrm{~A})$ & 499 & 245 & 6567 & 56 \\
\hline $\mathrm{H}(9 \mathrm{~B})$ & -301 & 170 & 6403 & 56 \\
\hline $\mathrm{H}(9 \mathrm{C})$ & 140 & 820 & 5784 & 56 \\
\hline $\mathrm{H}(11)$ & 2069 & -5256 & 1662 & 34 \\
\hline $\mathrm{H}(12)$ & 2762 & -7094 & 1419 & 40 \\
\hline $\mathrm{H}(13)$ & 3675 & -7649.0005 & 2279 & 43 \\
\hline $\mathrm{H}(14)$ & 3958 & -6243 & 3327 & 45 \\
\hline $\mathrm{H}(15)$ & 3313 & -4309 & 3524 & 36 \\
\hline $\mathrm{H}(17)$ & 767 & -2118 & 2806 & 30 \\
\hline $\mathrm{H}(18)$ & -273 & -2990 & 3065 & 35 \\
\hline $\mathrm{H}(19)$ & -365 & -5286 & 3412 & 35 \\
\hline $\mathrm{H}(20)$ & 564 & -6720 & 3461 & 33 \\
\hline $\mathrm{H}(21)$ & 1594 & -5876 & 3145 & 29 \\
\hline $\mathrm{H}(23)$ & 3328 & 84 & 675 & 41 \\
\hline $\mathrm{H}(24)$ & 3018 & 1541 & -349.0000 & 56 \\
\hline $\mathrm{H}(25)$ & 1948 & 2479 & -508 & 61 \\
\hline $\mathrm{H}(26)$ & 1175 & 2010 & 365 & 53 \\
\hline $\mathrm{H}(27)$ & 1481 & 585 & 1405 & 38 \\
\hline $\mathrm{H}(29)$ & 4013 & -875 & 2640 & 38 \\
\hline $\mathrm{H}(30)$ & 4988 & -2093 & 2443 & 49 \\
\hline $\mathrm{H}(31)$ & 4971 & -3728 & 1487 & 49 \\
\hline $\mathrm{H}(32)$ & 3985 & -4154 & 717 & 47 \\
\hline $\mathrm{H}(33)$ & 3007 & -2956 & 906 & 36 \\
\hline $\mathrm{H}(34 \mathrm{~A})$ & 3955 & -7177 & 487 & 137 \\
\hline $\mathrm{H}(34 \mathrm{~B})$ & 4412 & -6455 & 1165 & 137 \\
\hline $\mathrm{H}(35 \mathrm{~A})$ & 5080 & -6208 & 356 & 107 \\
\hline $\mathrm{H}(35 \mathrm{~B})$ & 4747 & -7410 & -172 & 107 \\
\hline $\mathrm{H}(36 \mathrm{~A})$ & 5784 & -7646 & 1022 & 73 \\
\hline $\mathrm{H}(36 \mathrm{~B})$ & 5665 & -8575 & 267 & 73 \\
\hline $\mathrm{H}(37 \mathrm{~A})$ & 4955 & -9972 & 801 & 72 \\
\hline $\mathrm{H}(37 \mathrm{~B})$ & 5286 & -9368 & 1595 & 72 \\
\hline
\end{tabular}

